\title{
Error estimates for a numerical approximation to the compressible barotropic Navier-Stokes equations
}

\author{
Thierry Gallouët Raphaèle Herbin David Maltese Antonin Novotny* \\ Aix-Marseille Université, CNRS, Centrale Marseille, I2M, UMR 7373, 13453 Marseille, France \\ and \\ IMATH, EA 2134, Université de Toulon, BP 20132, 83957 La Garde, France

\begin{abstract}
We present here a general method based on the investigation of the relative energy of the system, that provides an unconditional error estimate for the approximate solution of the barotropic Navier Stokes equations obtained by time and space discretization. We use this methodology to derive an error estimate for a specific DG/finite element scheme for which the convergence was proved in [27. This is an extended version of the paper submitted to IMAJNA.
\end{abstract}

Keywords: Compressible fluids, Navier-Stokes equations, Relative energy, Error estimates, Finite element mlethods, Finite volume methods

AMS classification 35Q30, 65N12, 65N30, 76N10, 76N15, 76M10, 76M12

\section{Contents}

1 Introduction

2 The continuous problem

3 The numerical scheme 6

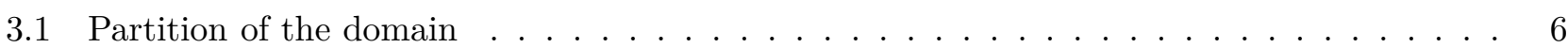

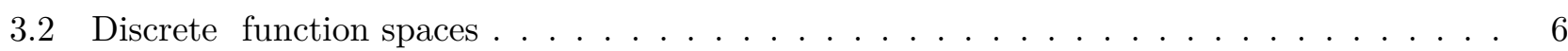

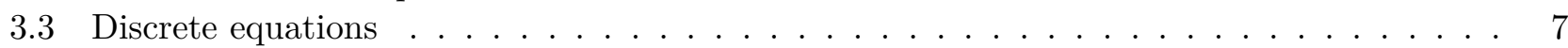

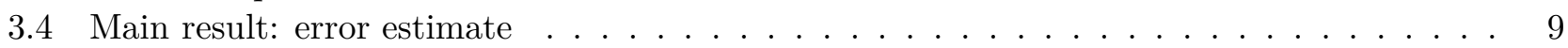

4 Mesh independent estimates 11

4.1 Energy Identity . . . . . . . . . . . . . . . . . . . . 11

4.2 Estimates . . . . . . . . . . . . . . . . . . . . . 14

5 Exact relative energy inequality for the discrete problem $\quad 16$

6 Approximate relative energy inequality for the discrete problem 19

$\begin{array}{lll}7 & \text { A discrete identity satisfied by the strong solution } & 26\end{array}$

8 End of the proof of the error estimate (Theorem 3.1 ) 33

\footnotetext{
${ }^{*}$ This work was supported by the MODTERCOM project within the APEX programme of the Provence-Alpes-Côte d'Azur region
} 


\section{1 - Introduction}

The aim of this paper is to derive an error estimate for approximate solutions of the compressible barotropic Navier-Stokes equations obtained by a discretisation scheme. These equations are posed on the time-space domain $Q_{T}=(0, T) \times \Omega$, where $\Omega$ is a bounded polyhedral domain of $\mathbb{R}^{d}, d=2,3$ and $T>0$, and read:

$$
\begin{aligned}
& \partial_{t} \varrho+\operatorname{div}(\varrho \boldsymbol{u})=0, \\
& \partial_{t}(\varrho \boldsymbol{u})+\operatorname{div}(\varrho \boldsymbol{u} \otimes \boldsymbol{u})-\mu \Delta \boldsymbol{u}-(\mu+\lambda) \nabla \operatorname{div} \boldsymbol{u}+\nabla_{x} p(\varrho)=\mathbf{0},
\end{aligned}
$$

supplemented with the initial conditions

$$
\varrho(0, x)=\varrho_{0}(x), \varrho \boldsymbol{u}(0, x)=\varrho_{0} \boldsymbol{u}_{0},
$$

where $\varrho_{0}$ and $\boldsymbol{u}_{0}$ are given functions from $\Omega$ to $\mathbb{R}_{+}$and $\mathbb{R}^{d}$ respectively, and boundary conditions

$$
\boldsymbol{u}_{\mid(0, T) \times \partial \Omega}=0 .
$$

In the above equations, the unknown functions are the scalar density field $\varrho(t, x) \geq 0$ and vector velocity field $\boldsymbol{u}=\left(u_{1}, \ldots, u_{d}\right)(t, x)$, where $t \in(0, T)$ denotes the time and $x \in \Omega$ is the space variable. The viscosity coefficients $\mu$ and $\lambda$ are such that

$$
\mu>0, \lambda+\frac{2}{d} \mu \geq 0
$$

The pressure $p$ is a given by an equation of state, that is a function of density which satisfies

$$
p \in C([0, \infty)) \cap C^{1}(0, \infty), p(0)=0, p^{\prime}(\varrho)>0 .
$$

In addition to (1.5), in the error analysis, we shall need to prescribe the asymptotic behavior of the pressure at large densities

$$
\lim _{\varrho \rightarrow \infty} \frac{p^{\prime}(\varrho)}{\varrho^{\gamma-1}}=p_{\infty}>0 \quad \text { with some } \gamma \geq 1 ;
$$

furthermore, if $\gamma<2$ in (1.6), we need the additional condition (for small densities):

$$
\liminf _{\varrho \rightarrow 0} \frac{p^{\prime}(\varrho)}{\varrho^{\alpha+1}}=p_{0}>0 \quad \text { with some } \alpha \leq 0 .
$$

The main underlying idea of this paper is to derive the error estimates for approximate solutions of problem (1.1)-(1.3) obtained by time and space discretization by using the discrete version of the relative energy method introduced on the continuous level in [12,13, 15. In spite of the fact that the relative energy method looks at the first glance pretty much similar to the widely used relative entropy method (and both approaches translate the same thermodynamic stability conditions), they are very different in appearance and formulation and may provide different results. The notions of relative entropy and relative entropy inequality were first introduced by Dafermos [7] in the context of systems of conservation laws and in particular for the compressible Euler equations. The relative energy functional was suggested and successfully used for the investigation of the stability of weak solutions to the equations of viscous compressible and heat conducting fluids in [13. In contrast with the relative entropy of Dafermos, for the viscous and heat conducting fluids, the relative energy approach is able to provide the structural stability of weak solutions, while the relative entropy approach fails in this case.

Both functionals coincide (modulo a change of variables) in the case of (viscous) compressible flows 
in the barotropic regime. The relative energy functional and the intrinsic version of the relative energy inequality have been recently employed to obtain several stability results for the weak solutions to these equations, including the weak strong uniqueness principle, see [12,15]. Note that particular versions of the relative entropy inequality with particular specific test functions had been previously derived in the context of low Mach number limits, see e.g. [32,34.

The discrete version of the Dafermos relative entropy was employed in the non viscous case to derive an error estimate for the numerical approximation to a hyperbolic system of conservation laws and, in particular, to the compressible Euler equations [4. In this latter paper, the authors assume an $L^{\infty}$ bound for the discrete solution, which is uniform with respect to the size of the space and time disretization (usually called stability hypothesis), that is not provided by the discrete equations. The same method with the same severe hypotheses have been used in [35] to treat the compressible Navier-Stokes equations. The error analysis in the present paper relies on the theoretical background introduced in [12] and yields an unconditional result; in particular, we do not need any assumed bound on the solution to get the error estimate.

The mathematical analysis of numerical schemes for the discretization of the steady and/or non steady compressible Navier-Stokes and/or compressible Stokes equations has been the object of some recent works. The convergence of the discrete solutions to the weak solutions of the compressible stationary Stokes was shown for a finite volume- non conforming P1 finite element [10, 11, 18] and for the wellknown MAC scheme which was introduced in 22 and is widely used in computational fluid dynamics (see e.g. [29]). The unsteady Stokes problem was also discretized by some other discretization schemes on a reformulation of the problem, which were proven to be convergent [24 26]. The unsteady barotropic Navier-Stokes equations was recently investigated in [27] in the case $\gamma>3$ (there is a real difficulty in the realistic case $\gamma \leq 3$ arising from the treatment of the non linear convective term). However, in these works, the rate of convergence is not provided; in fact, to the best of our knowledge, no error analysis has yet been performed for any of the numerical schemes that have been designed for the compressible Navier-Stokes equations, in spite of its great importance for the numerical analysis of the equations and for the mathematical simulations of compressible fluid flows. We present here a general technique to obtain an error analysis and apply it to one of the available numerical schemes. To the best of our knowledge, this is the first result of this type in the mathematical literature on the subject.

To achieve the goal, we systematically use the relative energy method on the discrete level. From this point of view, this paper is as valuable for the introduced methodology as for the result itself. Here, we apply the method to the scheme of [27. In spite of the fact that this latter scheme is not used in practice (see e.g. 28] for a related schemes used in industrial codes), we begin the error analysis with the scheme [27] because of its readily available convergence proof. In fact, we aim to use this approach to investigate the numerical errors of less academic numerical schemes, such as the finite volume - non conforming P1 finite element [17,20,21,28] or the MAC scheme [1,23].

The paper is organized as follows. After recalling the fundamental setting of the problem and the relative energy inequality in the continuous case in Section 2, we proceed in Section 3 to the discretization: we introduce the discrete functional spaces and the definition of the numerical scheme, and state the main result of the paper, that is the error estimate formulated in Theorem 3.1. The remaining sections are devoted to the proof of Theorem 3.1 ;

- In Section 4 we recall the existence theorem for the numerical scheme (Lemma 13.1) and derive estimates provided by the scheme.

- In Section 5, we derive the discrete intrinsic version of the relative energy inequality for the solutions of the numerical scheme (see Theorem [5.1). 
- The relative energy inequality is transformed to a more convenient form in Section 6, see Lemma 6.1 .

- Finally, in Section 7, we investigate the form of the discrete relative energy inequality with the test function being a strong solution to the original problem. This investigation is formulated in Lemma 7.1 and finally leads to a Gronwall type estimate formulated in Lemma 8.1. The latter yields the error estimates and finishes the proof of the main result.

Fundamental properties of the discrete functional spaces needed throughout the paper are reported in Appendix (Section 9). Some of them (especially those referring to the $L^{p}$ setting, $p \neq 2$ that are not currently available in the mathematical literature) are proved. Section 9 is therefore of the independent interest.

\section{2 - The continuous problem}

The aim of this section is to recall some fundamental notions and results. We begin by the definition of weak solutions to problem (1.1) $-(1.3)$.

Definition 2.1 (Weak solutions). Let $\varrho_{0}: \Omega \rightarrow[0,+\infty)$ and $\boldsymbol{u}_{0}: \Omega \rightarrow \mathbb{R}^{d}$ with finite energy $E_{0}=$ $\int_{\Omega}\left(\frac{1}{2} \varrho_{0}\left|\boldsymbol{u}_{0}\right|^{2}+H\left(\varrho_{0}\right)\right) \mathrm{d} x$ and finite mass $0<M_{0}=\int_{\Omega} \varrho_{0} \mathrm{~d} x$. We shall say that the pair $(\varrho, \boldsymbol{u})$ is a weak solution to the problem (1.1) -(1.3) emanating from the initial data $\left(\varrho_{0}, \boldsymbol{u}_{0}\right)$ if:

(a) $\varrho \in L^{\infty}\left(0, T ; L^{1}(\Omega)\right), \varrho \geq 0$ a.e. in $(0, T)$, and $\boldsymbol{u} \in L^{2}\left(0, T ; W_{0}^{1,2}(\Omega)\right)$.

(b) $\varrho \in C_{\text {weak }}\left([0, T] ; L^{1}(\Omega)\right)$, and the continuity equation (1.1a) is satisfied in the following weak sense

$$
\left.\int_{\Omega} \varrho \varphi \mathrm{d} x\right|_{0} ^{\tau}=\int_{0}^{\tau} \int_{\Omega}\left(\varrho \partial_{t} \varphi+\varrho \boldsymbol{u} \cdot \nabla_{x} \varphi\right) \mathrm{d} x \mathrm{dt}, \forall \tau \in[0, T], \forall \varphi \in C_{c}^{\infty}([0, T] \times \bar{\Omega}) .
$$

(c) $\varrho u \in C_{\text {weak }}\left([0, T] ; L^{1}(\Omega)\right)$, and the momentum equation $(\underline{1.1 b})$ is satisfied in the weak sense,

$$
\begin{aligned}
& \left.\int_{\Omega} \varrho \boldsymbol{u} \cdot \varphi \mathrm{d} x\right|_{0} ^{\tau}=\int_{0}^{\tau} \int_{\Omega}\left(\varrho \boldsymbol{u} \cdot \partial_{t} \varphi+\varrho \boldsymbol{u} \otimes \boldsymbol{u}: \nabla \varphi+p(\varrho) \operatorname{div} \varphi\right) \mathrm{d} x \mathrm{dt} \\
& \quad-\int_{0}^{\tau} \int_{\Omega}\left(\mu \nabla \boldsymbol{u}: \nabla_{x} \varphi \mathrm{d} x \mathrm{dt}+(\mu+\lambda) \operatorname{div} \boldsymbol{u} \operatorname{div} \varphi\right) \mathrm{d} x \mathrm{dt}, \forall \tau \in[0, T], \forall \varphi \in C_{c}^{\infty}\left([0, T] \times \Omega ; \mathbb{R}^{3}\right) .
\end{aligned}
$$

(d) The following energy inequality is satisfied

$$
\begin{gathered}
\left.\int_{\Omega}\left(\frac{1}{2} \varrho|\boldsymbol{u}|^{2}+H(\varrho)\right) \mathrm{d} x\right|_{0} ^{\tau}+\int_{0}^{\tau} \int_{\Omega}\left(\mu|\nabla \boldsymbol{u}|^{2}+(\mu+\lambda)|\operatorname{div} \boldsymbol{u}|^{2}\right) \mathrm{d} x \mathrm{dt} \leq 0, \text { for a.a. } \tau \in(0, T) \\
\text { with } H(\varrho)=\varrho \int_{1}^{\varrho} \frac{p(z)}{z^{2}} \mathrm{~d} z
\end{gathered}
$$

Here and hereafter the symbol $\left.\int_{\Omega} g \mathrm{~d} x\right|_{0} ^{\tau}$ is meant for $\int_{\Omega} g(\tau, x) \mathrm{d} x-\int_{\Omega} g_{0}(x) \mathrm{d} x$.

In the above definition, we tacitly assume that all the integrals in the formulas (2.1)-(2.3) are defined and we recall that $C_{\text {weak }}\left([0, T] ; L^{1}(\Omega)\right)$ is the space of functions of $L^{\infty}\left([0, T] ; L^{1}(\Omega)\right)$ which are continuous for the weak topology.

We notice that the function $\varrho \mapsto H(\varrho)$ is a solution of the ordinary differential equation $\varrho H^{\prime}(\varrho)-$ $H(\varrho)=p(\varrho)$ with the constant of integration fixed such that $H(1)=0$. 
Note that the existence of weak solutions emanating from the finite energy initial data is well-known on bounded Lipschitz domains under assumptions (1.5) and (1.6) provided $\gamma>d /(d-1)$, see Lions [30] for "large" values of $\gamma$, Feireisl and coauthors [14] for $\gamma>d /(d-1)$.

Let us now introduce the notion of relative energy. We first introduce the function

$$
\begin{aligned}
E: \quad & {[0, \infty) \times(0, \infty) \rightarrow \mathbb{R}, } \\
& (\varrho, r) \mapsto E(\varrho \mid r)=H(\varrho)-H^{\prime}(r)(\varrho-r)-H(r),
\end{aligned}
$$

where $H$ is defined by (2.4). Due to the monotonicity hypothesis in (1.5), $H$ is strictly convex on $[0, \infty)$, and therefore

$$
E(\varrho \mid r) \geq 0 \text { and } E(\varrho \mid r)=0 \Leftrightarrow \varrho=r .
$$

In order to measure a "distance" between a weak solution $(\varrho, \boldsymbol{u})$ of the compressible Navier-Stokes system and any other state $(r, \boldsymbol{U})$ of the fluid, we introduce the relative energy functional, defined by

$$
\mathcal{E}(\varrho, \boldsymbol{u} \mid r, \boldsymbol{U})=\int_{\Omega}\left(\frac{1}{2} \varrho|\boldsymbol{u}-\boldsymbol{U}|^{2}+E(\varrho \mid r)\right) \mathrm{d} x .
$$

It was proved recently in [12] that, provided assumption (1.5) holds, any weak solution satisfies the following so-called relative energy inequality

$$
\begin{aligned}
& \mathcal{E}(\varrho, \boldsymbol{u} \mid r, \boldsymbol{U})(\tau)-\mathcal{E}(\varrho, \boldsymbol{u} \mid r, \boldsymbol{U})(0)+\int_{0}^{\tau} \int_{\Omega}\left(\mu|\nabla(\boldsymbol{u}-\boldsymbol{U})|^{2}+(\mu+\lambda)|\operatorname{div}(\boldsymbol{u}-\boldsymbol{U})|^{2}\right) \mathrm{d} x \mathrm{dt} \\
& \leq \int_{0}^{\tau} \int_{\Omega}(\mu \nabla \boldsymbol{U}: \nabla(\boldsymbol{U}-\boldsymbol{u})+(\mu+\lambda) \operatorname{div} \boldsymbol{U} \operatorname{div}(\boldsymbol{U}-\boldsymbol{u})) \mathrm{d} x \mathrm{dt} \\
&+\int_{0}^{\tau} \int_{\Omega} \varrho \partial_{t} \boldsymbol{U} \cdot(\boldsymbol{U}-\boldsymbol{u}) \mathrm{d} x \mathrm{dt}+\int_{0}^{\tau} \int_{\Omega} \varrho \boldsymbol{u} \cdot \nabla \boldsymbol{U} \cdot(\boldsymbol{U}-\boldsymbol{u}) \mathrm{d} x \mathrm{dt} \\
&-\int_{0}^{\tau} \int_{\Omega} p(\varrho) \operatorname{div} \boldsymbol{U} \mathrm{d} x \mathrm{dt}+\int_{0}^{\tau} \int_{\Omega}(r-\varrho) \partial_{t} H^{\prime}(r) \mathrm{d} x \mathrm{dt}-\int_{0}^{\tau} \int_{\Omega} \varrho \nabla H^{\prime}(r) \cdot \boldsymbol{u} \mathrm{d} x \mathrm{dt}
\end{aligned}
$$

for a.a. $\tau \in(0, T)$, and for any pair of test functions

$$
r \in C^{1}([0, T] \times \bar{\Omega}), r>0, \boldsymbol{U} \in C^{1}\left([0, T] \times \bar{\Omega} ; \mathbb{R}^{3}\right),\left.\boldsymbol{U}\right|_{\partial \Omega}=0 .
$$

The stability of strong solutions in the class of weak solutions is stated in the following proposition.

Proposition 2.1 (Estimate on the relative energy). Let $\Omega$ be a Lipschitz domain. Assume that the viscosity coefficients satisfy assumptions (1.4), that the pressure $p$ is a twice continuously differentiable function on $(0, \infty)$ satisfying (1.5) and (1.6), and that $(\varrho, \boldsymbol{u})$ is a weak solution to problem (1.1) (1.3) emanating from initial data $\left(\varrho_{0} \geq 0, \boldsymbol{u}_{0}\right)$, with finite energy $E_{0}$ and finite mass $M_{0}=\int_{\Omega} \varrho_{0} \mathrm{~d} x>0$. Let $(r, \boldsymbol{U})$ in the class

$$
\left\{\begin{array}{l}
r \in C^{1}([0, T] \times \bar{\Omega}), 0<\underline{r}=\min _{(t, x) \in \bar{Q}_{T}} r(t, x) \leq r(t, x) \leq \bar{r}=\max _{(t, x) \in \bar{Q}_{T}} r(t, x), \\
\boldsymbol{U} \in C^{1}\left([0, T] \times \bar{\Omega} ; \mathbb{R}^{3}\right),\left.\boldsymbol{U}\right|_{(0, T) \times \partial \Omega}=0
\end{array}\right.
$$

be a (strong) solution of problem (1.1) emanating from the initial data $\left(r_{0}, \boldsymbol{U}_{0}\right)$. Then there exists

$$
c=c\left(T, \Omega, M_{0}, E_{0}, \underline{r}, \bar{r},\left|p^{\prime}\right|_{C^{1}([\underline{r}, \bar{r}])},\left\|\left(\nabla r, \partial_{t} r, \boldsymbol{U}, \nabla \boldsymbol{U}, \partial_{t} \boldsymbol{U}\right)\right\|_{L^{\infty}\left(Q_{T} ; \mathbb{R}^{19}\right)}\right)>0
$$

such that for almost all $t \in(0, T)$,

$$
\mathcal{E}(\varrho, \boldsymbol{u} \mid r, \boldsymbol{U})(t) \leq c \mathcal{E}\left(\varrho_{0}, \boldsymbol{u}_{0} \mid r_{0}, \boldsymbol{U}_{0}\right)
$$

This estimate (implying among others the weak-strong uniqueness) was proved in [12] (see also [15]) for pressure laws (1.6) with $\gamma>d /(d-1)$. It remains valid under weaker hypothesis on the pressure, such as (1.6) with $\gamma \geq 1$; this can be proved using ideas introduced in [2] and [31]. 


\section{3 - The numerical scheme}

\subsection{Partition of the domain}

We suppose that $\Omega$ is a bounded domain of $\mathbb{R}^{d}$, polygonal if $d=2$ and polyhedral if $d=3$. Let $\mathcal{T}$ be a decomposition of the domain $\Omega$ in tetrahedra, which we call hereafter a triangulation of $\Omega$, regardless of the space dimension. By $\mathcal{E}(K)$, we denote the set of the edges $(d=2)$ or faces $(d=3) \sigma$ of the element $K \in \mathcal{T}$ called hereafter faces, regardless of the dimension. The set of all faces of the mesh is denoted by $\mathcal{E}$; the set of faces included in the boundary $\partial \Omega$ of $\Omega$ is denoted by $\mathcal{E}_{\text {ext }}$ and the set of internal faces (i.e $\left.\mathcal{E} \backslash \mathcal{E}_{\text {ext }}\right)$ is denoted by $\mathcal{E}_{\text {int }}$. The triangulation $\mathcal{T}$ is assumed to be regular in the usual sense of the finite element literature (see e.g. [5]), and in particular, $\mathcal{T}$ satisfies the following properties:

- $\bar{\Omega}=\cup_{K \in \mathcal{T}} \bar{K}$

- if $(K, L) \in \mathcal{T}^{2}$, then $\bar{K} \cap \bar{L}=\emptyset$ or $\bar{K} \cap \bar{L}$ is a vertex or $\bar{K} \cap \bar{L}$ is a common face of $K$ and $L$; in the latter case it is denoted by $K \mid L$.

For each internal face of the mesh $\sigma=K \mid L, \boldsymbol{n}_{\sigma, K}$ stands for the normal vector of $\sigma$, oriented from $K$ to $L$ (so that $\boldsymbol{n}_{\sigma, K}=-\boldsymbol{n}_{\sigma, L}$ ). We denote by $|K|$ and $|\sigma|$ the ( $d$ and $d-1$ dimensional) Lebesgue measure of the tetrahedron $K$ and of the face $\sigma$ respectively, and by $h_{K}$ and $h_{\sigma}$ the diameter of $K$ and $\sigma$ respectively. We measure the regularity of the mesh thanks to the parameter $\theta$ defined by

$$
\theta=\inf \left\{\frac{\xi_{K}}{h_{K}}, K \in \mathcal{T}\right\}
$$

where $\xi_{K}$ stands for the diameter of the largest ball included in $K$. Last but not least we denote by $h$ the maximal size of the mesh,

$$
h=\max _{K \in \mathcal{T}} h_{K}
$$

The triangulation $\mathcal{T}$ is said to be regular if it satisfies

$$
\theta \geq \theta_{0}>0
$$

\subsection{Discrete function spaces}

Let $\mathcal{T}$ be a mesh of $\Omega$. We denote by $L_{h}(\Omega)$ the space of piecewise constant functions on the cells of the mesh; the space $L_{h}(\Omega)$ is the approximation space for the pressure and density. For $1 \leq p<\infty$, the mapping

$$
q \mapsto\|q\|_{L_{h}^{p}(\Omega)}=\|q\|_{L^{p}(\Omega)}=\left(\sum_{K \in \mathcal{T}}\left|K \| q_{K}\right|^{p}\right)^{1 / p}
$$

is a norm on $L_{h}(\Omega)$. We also introduce spaces of non-negative and positive functions:

$$
L_{h}^{+}(\Omega)=\left\{q \in L_{h}(\Omega), q_{K} \geq 0, \forall K \in \mathcal{T}\right\}, \quad L_{h}^{++}(\Omega)=\left\{q \in L_{h}(\Omega), q_{K}>0, \forall K \in \mathcal{T}\right\} .
$$

The approximation space for the velocity field is the space $\boldsymbol{W}_{h}(\Omega)=V_{h}\left(\Omega ; \mathbb{R}^{d}\right)$, where $V_{h}(\Omega)$ is the non conforming piecewise linear finite element space [6,8] defined by:.

$$
\begin{aligned}
& V_{h}(\Omega)=\left\{v \in L^{2}(\Omega), \quad \forall K \in \mathcal{T}, v_{\mid K}\right. \in \mathbb{P}_{1}(K), \\
&\left.\forall \sigma \in \mathcal{E}_{\text {int }}, \sigma=K \mid L, \int_{\sigma} v_{\mid K} \mathrm{~d} S=\int_{\sigma} v_{\mid L} \mathrm{~d} S, \quad \forall \sigma \in \mathcal{E}_{\text {ext }}, \int_{\sigma} v \mathrm{~d} S=0\right\},
\end{aligned}
$$

where $\mathbb{P}_{1}(K)$ denotes the space of affine functions on $K$ and $\mathrm{d} S$ the integration with respect to the $(d-1)$-dimensional Lebesgue measure on the face $\sigma$. Each element $v \in V_{h}(\Omega)$ can be written in the form

$$
v(x)=\sum_{\sigma \in \mathcal{E}_{\mathrm{int}}} v_{\sigma} \varphi_{\sigma}(x), \quad x \in \Omega
$$


where the set $\left\{\varphi_{\sigma}\right\}_{\sigma \in \mathcal{E}_{\text {int }}} \subset V_{h}(\Omega)$ is the classical basis determined by

$$
\forall\left(\sigma, \sigma^{\prime}\right) \in \mathcal{E}_{\text {int }}^{2}, \int_{\sigma^{\prime}} \varphi_{\sigma} \mathrm{d} S=\delta_{\sigma, \sigma^{\prime}}, \quad \forall \sigma^{\prime} \in \mathcal{E}_{\text {ext }}, \int_{\sigma^{\prime}} \varphi_{\sigma} \mathrm{d} S=0
$$

and $\left\{v_{\sigma}\right\}_{\sigma \in \mathcal{E}_{\text {int }}} \subset R$ is the set of degrees of freedom relative to $v$. Notice that $V_{h}(\Omega)$ approximates the functions with zero traces in the sense that for all elements in $V_{h}(\Omega), v_{\sigma}=0$ provided $\sigma \in \mathcal{E}_{\text {ext }}$. Since only the continuity of the integral over each face of the mesh is imposed, the functions in $V_{h}(\Omega)$ may be discontinuous through each face; the discretization is thus nonconforming in $W^{1, p}\left(\Omega ; \mathbb{R}^{d}\right), 1 \leq p \leq \infty$. Finally, we notice that for any $1 \leq p<\infty$ the expression

$$
|v|_{V_{h}^{p}(\Omega)}=\left(\sum_{K \in \mathcal{T}}\|\nabla v\|_{L^{p}\left(K ; \mathbb{R}^{d}\right)}^{p}\right)^{1 / p}
$$

is a norm on $V_{h}(\Omega)$ and we denote by $V_{h}^{p}(\Omega)$ the space $V_{h}(\Omega)$ endowed with this norm.

We finish this section by introducing some notations. For a function $v$ in $L^{1}(\Omega)$, we set

$$
v_{K}=\frac{1}{|K|} \int_{K} v \mathrm{~d} x \text { for } K \in \mathcal{T} \text { and } \hat{v}(x)=\sum_{K \in \mathcal{T}} v_{K} 1_{K}(x), x \in \Omega
$$

so that $\hat{v} \in L_{h}(\Omega)$. Here and in what follows, $1_{K}$ is the characteristic function of $K$.

If $v \in W^{1, p}(\Omega)$, we set

$$
v_{\sigma}=\frac{1}{|\sigma|} \int_{\sigma} v \mathrm{~d} S \text { for } \sigma \in \mathcal{E}
$$

Finally, if $v \in W_{0}^{1, p}(\Omega)$, we set

$$
v_{h}(x)=\sum_{\sigma \in \mathcal{E}_{\text {int }}} v_{\sigma} \varphi_{\sigma}(x), x \in \Omega .
$$

so that $v_{h} \in V_{h}(\Omega)$. In accordance with the above notation, for $v \in W_{0}^{1, p}(\Omega)$, the symbol $\hat{v}_{h}$ means $\widehat{v_{h}}(x)=\sum_{\sigma \in \mathcal{E}_{\text {int }}} v_{\sigma} \hat{\phi}_{\sigma}(x)$, and the symbol $v_{h, K}=\frac{1}{|K|} \int_{K} v_{h}(x) \mathrm{d} x$ and the symbol $\hat{v}_{h, \sigma}^{\text {up }}=\left[\widehat{\left(v_{h}\right)}\right]_{\sigma}^{\text {up }}$.

\subsection{Discrete equations}

Let us consider a partition $0=t_{0}<t_{1}<\ldots<t_{N}=T$ of the time interval $[0, T]$, which, for the sake of simplicity, we suppose uniform. Let $k$ be the constant time step $k=t_{n}-t_{n-1}$ for $n=1, \ldots, N$. The density field $\varrho\left(t_{n}, x\right)$ and the velocity field $\boldsymbol{u}\left(t_{n}, x\right)$ will be approximated by the quantities

$$
\varrho^{n}(x)=\sum_{K \in \mathcal{T}} \varrho_{K}^{n} 1_{K}(x), \quad \boldsymbol{u}^{n}(x)=\sum_{\sigma \in \mathcal{E}} \boldsymbol{u}_{\sigma}^{n} \varphi_{\sigma}(x),
$$

where the approximate densities $\left(\varrho_{K}^{n}\right)_{K \in \mathcal{T}, n=1, \ldots, N}$ and velocities $\left(\boldsymbol{u}_{\sigma}^{n}\right)_{\sigma \in \mathcal{E}_{\text {int }}, n=1, \ldots, N}$ are the discrete unknowns (with $\varrho_{K}^{n} \in \mathbb{R}^{+}$and $\boldsymbol{u}_{\sigma}^{n} \in \mathbb{R}^{d}$ ).

For the future convenience, we denote here and hereafter,

$$
\varrho(t, x)=\sum_{n=1}^{N} \varrho^{n}(x) 1_{[n-1, n)}(t), \quad \boldsymbol{u}(t, x)=\sum_{n=1}^{N} \boldsymbol{u}^{n}(x) 1_{[n-1, n)}(t)
$$

and recall that the usual Lebesgue norms of these functions read

$$
\|\varrho\|_{L^{\infty}\left(0, T ; L^{p}(\Omega)\right.} \equiv \max _{n=1, \ldots, N}\left\|\varrho^{n}\right\|_{L^{p}(\Omega)}, \quad\|\boldsymbol{u}\|_{L^{p}\left(0, T ; L^{q}\left(\Omega ; \mathbb{R}^{3}\right)\right.} \equiv k\left(\sum_{n=1}^{N}\left\|\boldsymbol{u}^{n}\right\|_{L^{q}\left(\Omega ; \mathbb{R}^{3}\right)}^{p}\right)^{1 / p}
$$

Starting from this point, unlike in Section 1, here and hereafter, the couple $(\varrho, \mathbf{u})$ respectively $\left(\varrho^{n}, \mathbf{u}^{n}\right)$ introduced in (3.10 3.12) denote always exclusively a discrete numerical solution. 
The numerical scheme consists in writing the equations that are solved to determine these discrete unknowns. In order to ensure the positivity of the approximate densities, we shall use an upwinding technique for the density in the mass equation. For $q \in L_{h}(\Omega)$ and $\boldsymbol{u} \in \mathbf{W}_{h}(\Omega)$, the upwinding of $q$ with respect to $\boldsymbol{u}$ is defined, for $\sigma=K \mid L \in \mathcal{E}_{\text {int }}$ by

$$
q_{\sigma}^{\text {up }}=\left\{\begin{array}{l}
q_{K} \text { if } \boldsymbol{u}_{\sigma} \cdot \boldsymbol{n}_{\sigma, K}>0 \\
q_{L} \text { if } \boldsymbol{u}_{\sigma} \cdot \boldsymbol{n}_{\sigma, K} \leq 0
\end{array}\right.
$$

so that

$$
\sum_{\sigma \in \mathcal{E}(K)} q_{\sigma}^{\mathrm{up}} \boldsymbol{u}_{\sigma} \cdot \mathbf{n}_{\sigma, K}=\sum_{\substack{\sigma \in \mathcal{E}(K) \\ \sigma=K \mid L}}\left(q_{K}\left[\boldsymbol{u}_{\sigma} \cdot \mathbf{n}_{\sigma, K}\right]^{+}-q_{L}\left[\boldsymbol{u}_{\sigma} \cdot \mathbf{n}_{\sigma, K}\right]^{-}\right),
$$

where $a^{+}=\max (a, 0), a^{-}=-\min (a, 0)$.

Let us then consider the following numerical scheme [27]:

Given $\left(\varrho^{0}, \boldsymbol{u}^{0}\right) \in L_{h}^{+}(\Omega) \times \boldsymbol{W}_{h}(\Omega)$ find $\left(\varrho^{n}\right)_{1 \leq n \leq N} \subset\left(L_{h}(\Omega)\right)^{N},\left(\boldsymbol{u}^{n}\right)_{1 \leq n \leq N} \subset\left(\boldsymbol{W}_{h}(\Omega)\right)^{N}$ such that for all $n=1, \ldots, N$

$$
\begin{aligned}
& |K| \frac{\varrho_{K}^{n}-\varrho_{K}^{n-1}}{k}+\sum_{\sigma \in \mathcal{E}(K)}|\sigma| \varrho_{\sigma}^{n, \mathrm{up}}\left[\boldsymbol{u}_{\sigma}^{n} \cdot \boldsymbol{n}_{\sigma, K}\right]=0, \quad \forall K \in \mathcal{T}, \\
& \sum_{K \in \mathcal{T}} \frac{|K|}{k}\left(\varrho_{K}^{n} \boldsymbol{u}_{K}^{n}-\varrho_{K}^{n-1} \boldsymbol{u}_{K}^{n-1}\right) \cdot \boldsymbol{v}_{K}+\sum_{K \in \mathcal{T}} \sum_{\sigma \in \mathcal{E}(K)}|\sigma| \varrho_{\sigma}^{n, \text { up }} \hat{\boldsymbol{u}}_{\sigma}^{n, \mathrm{up}}\left[\boldsymbol{u}_{\sigma}^{n} \cdot \boldsymbol{n}_{\sigma, K}\right] \cdot \boldsymbol{v}_{K} \\
& \quad-\sum_{K \in \mathcal{T}} p\left(\varrho_{K}^{n}\right) \sum_{\sigma \in \mathcal{E}(K)}|\sigma| \boldsymbol{v}_{\sigma} \cdot \mathbf{n}_{\sigma, K}+\mu \sum_{K \in \mathcal{T}} \int_{K} \nabla \boldsymbol{u}^{n}: \nabla \boldsymbol{v} \mathrm{d} x \\
& \quad+(\mu+\lambda) \sum_{K \in \mathcal{T}} \int_{K} \operatorname{div} \boldsymbol{u}^{n} \operatorname{div} \boldsymbol{v} \mathrm{d} x=0, \forall \boldsymbol{v} \in \boldsymbol{W}_{h}(\Omega) .
\end{aligned}
$$

Note that the boundary condition $\boldsymbol{u}_{\sigma}^{n}=0$ if $\sigma \in \mathcal{E}_{\text {ext }}$ is ensured by the definition of the space $V_{h}(\Omega)$. Note also that if $\sigma \in \mathcal{E}_{\text {int }}, \sigma=K \mid L$, one has, following (3.7) and (3.13),

$$
\hat{\boldsymbol{u}}_{\sigma}^{n, \text { up }}=u_{K}^{n}=\frac{1}{\mid K]} \int_{K} \boldsymbol{u}^{n}(x) \mathrm{d} x \text { if } \boldsymbol{u}_{\sigma}^{n} \cdot \boldsymbol{n}_{\sigma, K}>0 \text { and } \hat{\boldsymbol{u}}_{\sigma}^{n, \text { up }}=u_{L}^{n}=\frac{1}{\mid L]} \int_{L} \boldsymbol{u}^{n}(x) \mathrm{d} x \text { if } \boldsymbol{u}_{\sigma}^{n} \cdot \boldsymbol{n}_{\sigma, K}<0 .
$$

It is well known that any solution $\left(\varrho^{n}\right)_{1 \leq n \leq N} \subset\left(L_{h}(\Omega)\right)^{N}$ satisfies $\varrho^{n}>0$ thanks to the upwind choice in (3.14a) (see e.g. [17, 27]). Furthermore, summing (3.14a) over $K \in \mathcal{T}$ immediately yields the total conservation of mass, which reads:

$$
\forall n=1, \ldots N, \quad \int_{\Omega} \varrho^{n} \mathrm{~d} x=\int_{\Omega} \varrho^{0} \mathrm{~d} x .
$$

We finally state in this section the existence result, which can be proved by a topological degree argument, [17,27].

Proposition 3.1 (Existence). Let $\left(\varrho^{0}, \boldsymbol{u}^{0}\right) \in L_{h}^{++}(\Omega) \times \boldsymbol{W}_{h}(\Omega)$. Under assumptions (1.4) and (1.5), Problem (3.14) admits at least one solution

$$
\left(\varrho^{n}\right)_{1 \leq n \leq N} \in\left[L_{h}^{++}(\Omega)\right]^{N},\left(\boldsymbol{u}^{n}\right)_{1 \leq n \leq N} \in\left[\boldsymbol{W}_{h}(\Omega)\right]^{N} .
$$




\subsection{Main result: error estimate}

Let $(r, \boldsymbol{U}):[0, T] \times \bar{\Omega} \mapsto(0, \infty) \times \mathbb{R}^{3}$ be $C^{2}$ functions such that $\boldsymbol{U}=\mathbf{0}$ on $\partial \Omega$. Let $(\varrho, \boldsymbol{u})$ be a solution of the discrete problem (3.14). Inspired by (2.6), we introduce the discrete relative energy functional

$$
\begin{aligned}
\mathcal{E}\left(\varrho^{n}, \boldsymbol{u}^{n} \mid r^{n}, \boldsymbol{U}^{n}\right) & =\int_{\Omega}\left(\frac{1}{2} \varrho^{n}\left|\hat{\boldsymbol{u}}^{n}-\hat{\boldsymbol{U}}_{h}^{n}\right|^{2}+E\left(\varrho^{n} \mid \hat{r}^{n}\right)\right) \mathrm{d} x \\
& =\sum_{K \in \mathcal{T}}|K|\left(\frac{1}{2} \varrho_{K}\left|\boldsymbol{u}_{K}^{n}-\boldsymbol{U}_{h, K}^{n}\right|^{2}+E\left(\varrho_{K}^{n} \mid r_{K}^{n}\right)\right),
\end{aligned}
$$

where

$$
r^{n}(x)=r\left(t_{n}, x\right), \boldsymbol{U}^{n}(x)=\boldsymbol{U}\left(t_{n}, x\right), n=0, \ldots, N,
$$

$\left(\varrho^{n}, \boldsymbol{u}^{n}\right)$ is defined in ( $(3.10)$, and $E$ is defined by (2.5). Let us finally introduce the notations

$$
M_{0}=\sum_{K \in \mathcal{K}}|K| \varrho_{K}^{0} \text {, and } E_{0}=\sum_{K \in \mathcal{K}}|K|\left(\frac{1}{2} \varrho_{K}^{0}\left|\boldsymbol{u}_{K}^{0}\right|^{2}+H\left(\varrho_{K}^{0}\right)\right) .
$$

Now, we are ready to state the main result of this paper. For the sake of clarity, we shall state the theorem and perform the proofs only in the most interesting three dimensional case. The modifications to be done for the two dimensional case, which is in fact more simple, are mostly due to the different Sobolev embedings, and are left to the interested reader.

Theorem 3.1 (Error estimate). Let $\theta_{0}>0$ and $\mathcal{T}$ be a regular triangulation of a bounded polyhedral domain $\Omega \subset \mathbb{R}^{3}$ introduced in Section [3.1 such that $\theta \geq \theta_{0}$, where $\theta$ is defined in (3.1). Let $p$ be a twice continuously differentiable function satisfying assumptions (1.5), (1.6) with $\gamma \geq 3 / 2$, and the additional assumption (1.7) in the case $\gamma<2$. Let the viscosity coefficients satisfy assumptions (1.4). Suppose that $\left(\varrho^{0}, \boldsymbol{u}^{0}\right) \in L_{h}^{+}(\Omega) \times \boldsymbol{W}_{h}(\Omega)$ and that $\left(\varrho^{n}\right)_{1 \leq n \leq N} \subset\left[L_{h}^{+}(\Omega)\right]^{N},\left(\boldsymbol{u}^{n}\right)_{1 \leq n \leq N} \subset\left[\boldsymbol{W}_{h}(\Omega)\right]^{N}$ is a solution of the discrete problem (3.14). Let $(r, \boldsymbol{U})$ in the class

$$
\begin{aligned}
& r \in C^{2}([0, T] \times \bar{\Omega}), \quad 0<\underline{r}:=\min _{(t, x) \in \bar{Q}_{T}} \leq r(t, x) \leq \bar{r}:=\max _{(t, x) \in \bar{Q}_{T}} r(t, x), \\
& \boldsymbol{U} \in C^{2}\left([0, T] \times \bar{\Omega} ; \mathbb{R}^{3}\right),\left.\boldsymbol{U}\right|_{\partial \Omega}=0
\end{aligned}
$$

be a (strong) solution of problem (1.1). Then there exists

$$
\begin{aligned}
c=c\left(T,|\Omega|, \operatorname{diam}(\Omega), \theta_{0}, \gamma, M_{0}, E_{0}, \underline{r}, \bar{r}\right. \\
\left.\qquad\left|p^{\prime}\right|_{C^{1}([\underline{r}, \bar{r}])},\left\|\left(\nabla r, \partial_{t} r, \partial_{t} \nabla r, \partial_{t}^{2} r, \boldsymbol{U}, \nabla \boldsymbol{U}, \nabla^{2} \boldsymbol{U}, \partial_{t} \boldsymbol{U}, \partial_{t}^{2} \boldsymbol{U}, \partial_{t} \nabla \boldsymbol{U}\right)\right\|_{L^{\infty}\left(Q_{T} ; \mathbb{R}^{68}\right)}\right) \in(0,+\infty)
\end{aligned}
$$

(independent of $h, k$ ) such that for any $m=1, \ldots, N$,

$$
\mathcal{E}\left(\varrho^{m}, \boldsymbol{u}^{m} \mid r^{m}, \boldsymbol{U}^{m}\right)+k \sum_{n=1}^{m} \sum_{K \in \mathcal{T}} \int_{K}\left|\nabla_{x}\left(u^{n}-\mathbf{U}_{h}^{n}\right)\right|^{2} \mathrm{dx} \leq c\left(\mathcal{E}\left(\varrho^{0}, \boldsymbol{u}^{0} \mid r^{0}, \boldsymbol{U}^{0}\right)+h^{A}+\sqrt{k}\right),
$$

where

$$
A= \begin{cases}\frac{2 \gamma-3}{\gamma} & \text { if } \gamma \in(3 / 2,2], \\ 1 / 2 & \text { if } \gamma>2 .\end{cases}
$$

Starting from this point, unlike in Section 1 , here and hereafter, the symbol $\mathcal{E}$ refers always to the discrete relative energy functional defined in (3.16). 


\section{Remark 3.1.}

Assumptions (3.18) on the regularity of the strong solution $(r, \mathbf{U})$ in Theorem 3.1 may be slightly relaxed: It is enough to suppose

$$
\begin{gathered}
(r, \mathbf{U}) \in C^{1}\left([0, T] \times \bar{\Omega} ; \mathbb{R}^{4}\right), \nabla^{2} \mathbf{U} \in C\left([0, T] \times \bar{\Omega} ; \mathbb{R}^{3}\right), 0<\inf _{(t, x) \in \bar{Q}_{T}} r(t, x), \\
\partial_{t}^{2} r \in L^{1}\left(0, T ; L^{\gamma^{\prime}}(\Omega)\right), \partial_{t} \nabla r \in L^{2}\left(0, T ; L^{6 \gamma /(5 \gamma-6)}\left(\Omega ; \mathbb{R}^{3}\right)\right),\left(\partial_{t}^{2} \mathbf{U}, \partial_{t} \nabla \mathbf{U}\right) \in L^{2}\left(0, T ; L^{6 / 5}\left(\Omega ; \mathbb{R}^{12}\right)\right) .
\end{gathered}
$$

The constant in the error estimate depends on $\underline{r}$ and the norms of $r$ and $\mathbf{U}$ in these spaces. This improvement is at the price of more technicalities in estimates of several residual terms, namely in estimates (6.36.5), (6.14), (6.22), (7.9), (7.11 (7.13) and (8.3).

\section{Remark 3.2.}

1. Theorem 3.1 holds also for two dimensional bounded polygonal domains under the assumption that $\gamma \geq 1$. Assumption (1.7) on the asymptotic behavior of pressure near 0 is no more necessary in this case. The value of $A$ in the error estimate (3.19) is

$$
A=\left\{\begin{aligned}
\frac{2 \gamma-2}{\gamma} & \text { if } \gamma \in(1,2] \\
1 & \text { if } \gamma>2
\end{aligned}\right.
$$

2. Suppose that the discrete initial data $\left(\varrho^{0}, \mathbf{u}^{0}\right)$ coincide with the projection $\left(\hat{r}^{0}, \hat{\mathbf{U}}_{h}^{0}\right)$ of the initial data determining the strong solution. Then formula (3.19) provides in terms of classical Lebesgue spaces the following bounds:

$$
\left\|\varrho^{m}-r^{m}\right\|_{L^{2}\left(\Omega \cap\left\{\underline{r} / 2 \leq \varrho^{m} \leq 2 \bar{r}\right\}\right)}^{2}+\left\|\hat{\mathbf{u}}^{m}-\mathbf{U}^{m}\right\|_{L^{2}\left(\Omega \cap\left\{\underline{r} / 2 \leq \varrho^{m} \leq 2 \bar{r}\right\}\right)}^{2} \leq c\left(h^{A}+\sqrt{k}\right)
$$

for the "essential part" of the solution (where the numerical density remains bounded from above and from below outside zero), and

$$
\left|\left\{\varrho^{m} \leq \underline{r} / 2\right\}\right|+\left|\left\{\varrho^{m} \geq 2 \bar{r}\right\}\right|+\left\|\varrho^{m}\right\|_{L^{\gamma}\left(\Omega \cap\left\{\varrho^{m} \geq 2 \bar{r}\right\}\right)}^{\gamma}+\left\|\varrho^{m}\left|\hat{\mathbf{u}}^{m}-\mathbf{U}^{m}\right|^{2}\right\|_{L^{1}\left(\Omega \cap\left\{\varrho^{m} \geq 2 \bar{r}\right\}\right)} \leq c\left(h^{A}+\sqrt{k}\right)
$$

for the "residual part" of the solution, where the numerical density can be "close" to zero or infinity. (In the above formula, for $B \subset \Omega,|B|$ denotes the Lebesgue measure of B.)

Moreover, in the particular case of $p(\varrho)=\varrho^{2}$ (that however represents a non physical situation) $E(\varrho \mid r)=(\varrho-r)^{2}$ and the error estimate (3.19) reads

$$
\left\|\varrho^{m}-r^{m}\right\|_{L^{2}(\Omega)}^{2}+\left\|\varrho^{m}\left|\hat{\mathbf{u}}^{m}-\mathbf{U}^{m}\right|^{2}\right\|_{L^{1}(\Omega)} \leq c(\sqrt{h}+\sqrt{k})
$$

3. Theorem 3.1 can be viewed as a discrete version of Proposition [2.1. It is to be noticed that the assumptions on the constitutive law for pressure guaranteeing the error estimates for the scheme (3.14) are somewhat stronger $(\gamma \geq 3 / 2)$ than the assumptions needed for the stability in the continuous case $(\gamma \geq 1)$. The threshold value $\gamma=3 / 2$ is however in accordance with the existence theory of weak solutions. The assumptions on the regularity of the strong solution to be compared with the discrete solution in the scheme are slightly stronger than those needed to establish the stability estimates in the continuous case.

4. If $d=3$, we notice that the assumptions on the pressure (as function of the density) in Theorem 3.1 are compatible with the isentropic case $p(\varrho)=\varrho^{\gamma}$ for all values $\gamma \geq 3 / 2$. 
5. The scheme [27] contains in addition artificial stabilizing terms both in the continuity and momentum equations. These terms are necessary for the convergence proof in [27] even for the large values of $\gamma$. It is to be noticed that the error estimate in Theorem 3.1 is formulated for the numerical scheme without these stabilizing terms. Of course similar error estimate is a fortiori valid also for the scheme with the stabilizing terms, however, this issue is not discussed in the present paper.

The rest of the paper is devoted to the proof of Theorem 3.1. For the sake of simplicity, and in order to simplify notation, we present the proof for the uniformly regular mesh meaning that there exist positive numbers $c_{i}=c_{i}\left(\theta_{0}\right)$ such that

$$
c_{1} h_{K} \leq h \leq c_{2} h_{\sigma} \leq c_{3} h_{K}, \quad c_{1}|K| \leq|\sigma| h \leq c_{2}|\sigma| h_{K} \leq c_{3}|\sigma| h_{\sigma} \leq c_{4}|K|
$$

for any $K \in \mathcal{T}$ and any $\sigma \in \mathcal{E}$. The necessary (small) modifications needed to accommodate the regular mesh satisfying only (3.3) are straightforward. Even with this simplification the proof is quite involved, and some details have to be necessarily omitted to keep its length within reasonable bounds. The reader can eventually find them in the extended version of this paper available on ArXiv [19].

\section{4 - Mesh independent estimates}

We start by a remark on the notation. From now on, the letter $c$ denotes positive numbers that may tacitly depend on $T,|\Omega| \operatorname{diam}(\Omega), \gamma, \alpha, \theta_{0}, \lambda$ and $\mu$, and on other parameters; the dependency on these other parameters (if any) is always explicitly indicated in the arguments of these numbers. These numbers can take different values even in the same formula. They are always independent of the size of the discretisation $k$ and $h$.

\subsection{Energy Identity}

Our analysis starts with an energy inequality, which is crucial both in the convergence analysis and in the error analysis. We recall this energy estimate which is already given in [27], along with its proof for the sake of completeness.

Lemma 4.1. Let $\left(\varrho^{0}, \boldsymbol{u}^{0}\right) \in L_{h}^{+}(\Omega) \times \boldsymbol{W}_{h}(\Omega)$ and suppose that $\left(\varrho^{n}\right)_{1 \leq n \leq N} \in\left[L_{h}^{+}(\Omega)\right]^{N},\left(\boldsymbol{u}^{n}\right)_{1 \leq n \leq N} \in$ $\left[\boldsymbol{W}_{h}(\Omega)\right]^{N}$ is a solution of the discrete problem (3.14) with the pressure $p$ satisfying condition (1.5). Then there exist

$$
\begin{aligned}
& \varrho_{\sigma}^{n} \in\left[\min \left(\varrho_{K}^{n}, \varrho_{L}^{n}\right), \max \left(\varrho_{K}^{n}, \varrho_{L}^{n}\right)\right], \sigma=K \mid L \in \mathcal{E}_{\text {int }}, n=1, \ldots, N \\
& \varrho_{K}^{n-1, n} \in\left[\min \left(\varrho_{K}^{n-1}, \varrho_{K}^{n}\right), \max \left(\varrho_{K}^{n-1}, \varrho_{K}^{n}\right)\right], K \in \mathcal{T}, n=1, \ldots, N
\end{aligned}
$$

such that

$$
\begin{aligned}
\sum_{K \in \mathcal{T}}|K|\left(\frac{1}{2} \varrho_{K}^{m}\left|\boldsymbol{u}_{K}^{m}\right|^{2}+\right. & \left.H\left(\varrho_{K}^{m}\right)\right)-\sum_{K \in \mathcal{T}}|K|\left(\frac{1}{2} \varrho_{K}^{0}\left|\boldsymbol{u}_{K}^{0}\right|^{2}+H\left(\varrho_{K}^{0}\right)\right) \\
& +k \sum_{n=1}^{m} \sum_{K \in \mathcal{T}}\left(\mu \int_{K}\left|\nabla_{x} \boldsymbol{u}^{n}\right|^{2} \mathrm{~d} x+(\mu+\lambda) \int_{K}\left|\operatorname{div} \boldsymbol{u}^{n}\right|^{2} \mathrm{~d} x\right) \\
& +\left[D_{\text {time }}^{m,|\Delta \boldsymbol{u}|}\right]+\left[D_{\text {time }}^{m,|\Delta \varrho|}\right]+\left[D_{\text {space }}^{m,|\Delta \boldsymbol{u}|}\right]+\left[D_{\text {space }}^{m, \mid \Delta \varrho}\right]=0
\end{aligned}
$$


for all $m=1, \ldots, N$, where

$$
\begin{aligned}
& {\left[D_{\text {time }}^{m,|\Delta \boldsymbol{u}|}\right]=\sum_{n=1}^{m} \sum_{K \in \mathcal{T}}|K| \varrho_{K}^{n-1} \frac{\left|\boldsymbol{u}_{K}^{n}-\boldsymbol{u}_{K}^{n-1}\right|^{2}}{2}} \\
& {\left[D_{\text {time }}^{m,|\Delta \varrho|}\right]=\sum_{n=1}^{m} \sum_{K \in \mathcal{T}}|K| H^{\prime \prime}\left(\varrho_{K}^{n-1, n}\right) \frac{\left|\varrho_{K}^{n}-\varrho_{K}^{n-1}\right|^{2}}{2},} \\
& {\left[D_{\text {space }}^{m,|\Delta u|}\right]=k \sum_{n=1}^{m} \sum_{\sigma=K \mid L \in \mathcal{E}_{\text {int }}}|\sigma| \varrho_{\sigma}^{n, \text { up }} \frac{\left(\boldsymbol{u}_{K}^{n}-\boldsymbol{u}_{L}^{n}\right)^{2}}{2}\left|\boldsymbol{u}_{\sigma}^{n} \cdot \boldsymbol{n}_{\sigma, K}\right|,} \\
& {\left[D_{\text {space }}^{m,|\Delta \varrho|}\right]=k \sum_{n=1}^{m} \sum_{\sigma=K \mid L \in \mathcal{E}_{\text {int }}}|\sigma| H^{\prime \prime}\left(\varrho_{\sigma}^{n}\right) \frac{\left(\varrho_{K}^{n}-\varrho_{L}^{n}\right)^{2}}{2}\left|\boldsymbol{u}_{\sigma}^{n} \cdot \mathbf{n}_{\sigma, K}\right| .}
\end{aligned}
$$

Proof. Mimicking the formal derivation of the total energy conservation in the continuous case we take as test function $\boldsymbol{v}=\boldsymbol{u}^{n}$ in the discrete momentum equation (3.14b $)^{n}$ and obtain

$$
I_{1}+I_{2}+I_{3}+I_{4}=0
$$

where

$$
\begin{aligned}
& I_{1}=\sum_{K \in \mathcal{T}} \frac{|K|}{k}\left(\varrho_{k}^{n} \boldsymbol{u}_{K}^{n}-\varrho_{K}^{n-1} \boldsymbol{u}_{K}^{n-1}\right) \cdot \boldsymbol{u}_{K}^{n}, \quad I_{2}=\sum_{K \in \mathcal{T}} \sum_{\substack{\sigma \in \mathcal{E}(K) \\
\sigma=K \mid L}}|\sigma| \varrho_{\sigma}^{n, \text { up }} \hat{\boldsymbol{u}}_{\sigma}^{n, \mathrm{up}} \cdot \boldsymbol{u}_{K}^{n}\left[\boldsymbol{u}_{\sigma}^{n} \cdot \boldsymbol{n}_{\sigma, K}\right], \\
& I_{3}=-\sum_{K \in \mathcal{T}} \sum_{\substack{\sigma \in \mathcal{E}(K) \\
\sigma=K \mid L}}|\sigma| p\left(\varrho_{K}^{n}\right)\left[\boldsymbol{u}_{\sigma}^{n} \cdot \mathbf{n}_{\sigma, K}\right], \quad I_{4}=\sum_{K \in \mathcal{T}} \int_{K}\left(\mu \nabla \boldsymbol{u}^{n}: \nabla \boldsymbol{u}^{n}+(\mu+\lambda) \operatorname{div} \boldsymbol{u}^{n} \operatorname{div} \boldsymbol{u}^{n}\right) \mathrm{d} x
\end{aligned}
$$

Next, we multiply the continuity equation $(\underline{3.14 \mathrm{a}})_{K}^{n}$ by $\frac{1}{2}\left|\boldsymbol{u}_{K}^{n}\right|^{2}$ and sum over all $K \in \mathcal{T}$. We get

$$
I_{5}+I_{6}=0
$$

$$
\text { with } I_{5}=-\sum_{K \in \mathcal{T}} \frac{1}{2} \frac{|K|}{k}\left(\varrho_{K}^{n}-\varrho_{K}^{n-1}\right)\left|\boldsymbol{u}_{K}^{n}\right|^{2} \text { and } I_{6}=-\sum_{\substack{K \in \mathcal{T} \\ \sigma \in \mathcal{E}(K) \\ \sigma=K \mid L}} \frac{1}{2}|\sigma| \varrho_{\sigma}^{n, \mathrm{up}}\left[\boldsymbol{u}_{\sigma}^{n} \cdot \boldsymbol{n}_{\sigma, K}\right]\left|\boldsymbol{u}_{K}^{n}\right|^{2} .
$$

Finally, we multiply the continuity equation $(3.14 \mathrm{a})_{K}^{n}$ by $H^{\prime}\left(\varrho_{K}^{n}\right)$ and sum over all $K \in \mathcal{T}$. We obtain

$$
I_{7}+I_{8}=0
$$

$$
\text { with } I_{7}=\sum_{K \in \mathcal{T}} \frac{|K|}{k}\left(\varrho_{K}^{n}-\varrho_{K}^{n-1}\right) H^{\prime}\left(\varrho_{K}^{n}\right) \text { and } I_{8}=\sum_{K \in \mathcal{T}} \sum_{\substack{\sigma \in \mathcal{E}(K) \\ \sigma=K \mid L}}|\sigma| \varrho_{\sigma}^{n \text {,up }}\left[\boldsymbol{u}_{\sigma}^{n} \cdot \boldsymbol{n}_{\sigma, K}\right] H^{\prime}\left(\varrho_{K}^{n}\right) \text {. }
$$

We now sum formulas (4.3)-(4.5) in several steps.

Step 1: Term $I_{1}+I_{7}$. We verify by a direct calculation that

$$
I_{1}=\sum_{K \in \mathcal{T}} \frac{|K|}{k}\left(\frac{1}{2} \varrho_{K}^{n}\left|\boldsymbol{u}_{K}^{n}\right|^{2}-\frac{1}{2} \varrho_{K}^{n-1}\left|\boldsymbol{u}_{K}^{n-1}\right|^{2}\right)+\sum_{K \in \mathcal{T}} \frac{|K|}{k} \varrho_{K}^{n-1} \frac{\left|\boldsymbol{u}_{K}^{n}-\boldsymbol{u}_{K}^{n-1}\right|^{2}}{2} .
$$

In order to transform the term $I_{7}$, we employ the Taylor formula

$$
H^{\prime}\left(\varrho_{K}^{n}\right)\left(\varrho_{K}^{n}-\varrho_{K}^{n-1}\right)=H\left(\varrho_{K}^{n}\right)-H\left(\varrho_{K}^{n-1}\right)+\frac{1}{2} H^{\prime \prime}\left(\varrho_{K}^{n-1, n}\right)\left(\varrho_{K}^{n}-\varrho_{K}^{n-1}\right)^{2},
$$


where $\varrho_{K}^{n-1, n} \in\left[\min \left(\varrho_{K}^{n-1}, \varrho_{K}^{n}\right), \max \left(\varrho_{K}^{n-1}, \varrho_{K}^{n}\right)\right]$. Consequently,

$$
\begin{aligned}
I_{1}+I_{7}=\sum_{K \in \mathcal{T}} \frac{|K|}{k}\left(\frac{1}{2} \varrho_{K}^{n}\left|\boldsymbol{u}_{K}^{n}\right|^{2}\right. & \left.-\frac{1}{2} \varrho_{K}^{n-1}\left|\boldsymbol{u}_{K}^{n-1}\right|^{2}\right)+\sum_{K \in \mathcal{T}} \frac{|K|}{k}\left(H\left(\varrho_{K}^{n}\right)-H\left(\varrho_{K}^{n-1}\right)\right) \\
& +\sum_{K \in \mathcal{T}} \frac{|K|}{k} \varrho_{K}^{n-1} \frac{\left|\boldsymbol{u}_{K}^{n}-\boldsymbol{u}_{K}^{n-1}\right|^{2}}{2}+\sum_{K \in \mathcal{T}} \frac{|K|}{k} H^{\prime \prime}\left(\varrho_{K}^{n-1, n}\right) \frac{\left|\varrho_{K}^{n}-\varrho_{K}^{n-1}\right|^{2}}{2} .
\end{aligned}
$$

Step 2: Term $I_{2}+I_{6}$. The contribution of the face $\sigma=K \mid L$ to the sum $I_{2}+I_{6}$ reads, by virtue of (3.13),

$$
\begin{aligned}
|\sigma|\left[\boldsymbol{u}_{\sigma}^{n} \cdot \boldsymbol{n}_{\sigma, K}\right]^{+} \varrho_{K}\left(\left|\boldsymbol{u}_{K}^{n}\right|^{2}-\boldsymbol{u}_{K}^{n} \cdot \boldsymbol{u}_{L}^{n}-\frac{1}{2}\left|\boldsymbol{u}_{K}^{n}\right|^{2}+\frac{1}{2}\left|\boldsymbol{u}_{L}^{n}\right|^{2}\right) & \\
& +|\sigma|\left[\boldsymbol{u}_{\sigma}^{n} \cdot \boldsymbol{n}_{\sigma, L}\right]^{+} \varrho_{L}\left(\left|\boldsymbol{u}_{L}^{n}\right|^{2}-\boldsymbol{u}_{K}^{n} \cdot \boldsymbol{u}_{L}^{n}-\frac{1}{2}\left|\boldsymbol{u}_{L}^{n}\right|^{2}+\frac{1}{2}\left|\boldsymbol{u}_{K}^{n}\right|^{2}\right) .
\end{aligned}
$$

Consequently,

$$
I_{2}+I_{6}=\sum_{\sigma=K \mid L \in \mathcal{E}_{\mathrm{int}}}|\sigma|\left|\boldsymbol{u}_{\sigma}^{n} \cdot \boldsymbol{n}_{\sigma, K}\right| \varrho_{\sigma}^{n, \text { up }} \frac{\left(\boldsymbol{u}_{K}^{n}-\boldsymbol{u}_{L}^{n}\right)^{2}}{2} .
$$

Step 3: Term $I_{3}+I_{8}$. We have

$$
\begin{aligned}
& I_{8}=\sum_{K \in \mathcal{T}} \sum_{\substack{\sigma \in \mathcal{E}(K) \\
\sigma=K \mid L}}|\sigma|\left[\boldsymbol{u}_{\sigma}^{n} \cdot \boldsymbol{n}_{\sigma, K}\right]\left(H^{\prime}\left(\varrho_{K}^{n}\right)\left(\varrho_{\sigma}^{n, \text { up }}-\varrho_{K}^{n}\right)+H\left(\varrho_{K}^{n}\right)\right) \\
& +\sum_{K \in \mathcal{T}} \sum_{\substack{\sigma \in \mathcal{E}(K) \\
\sigma=K \mid L}}|\sigma|\left[\boldsymbol{u}_{\sigma}^{n} \cdot \boldsymbol{n}_{\sigma, K}\right]\left(\varrho_{K}^{n} H^{\prime}\left(\varrho_{K}^{n}\right)-H\left(\varrho_{K}^{n}\right)\right) .
\end{aligned}
$$

Recalling (3.13), we may write the contribution of the face $\sigma=K \mid L$ to the first sum in $I_{8}$; it reads

$$
\begin{aligned}
|\sigma|\left[\boldsymbol{u}_{\sigma}^{n} \cdot \boldsymbol{n}_{\sigma, K}\right]^{+}\left(H\left(\varrho_{K}^{n}\right)-H^{\prime}\left(\varrho_{L}^{n}\right)\left(\varrho_{K}^{n}-\varrho_{L}^{n}\right)\right. & \left.-H\left(\varrho_{L}^{n}\right)\right) \\
& +|\sigma|\left[\boldsymbol{u}_{\sigma}^{n} \cdot \boldsymbol{n}_{\sigma, L}\right]^{+}\left(H\left(\varrho_{L}^{n}\right)-H^{\prime}\left(\varrho_{K}^{n}\right)\left(\varrho_{L}^{n}-\varrho_{K}^{n}\right)-H\left(\varrho_{K}^{n}\right)\right) .
\end{aligned}
$$

Recalling that $r H^{\prime}(r)-H(r)=p(r)$, we get, employing the Taylor formula

$$
I_{3}+I_{8}=\sum_{\sigma=K \mid L \in \mathcal{E}_{\mathrm{int}}}\left|\boldsymbol{u}_{\sigma}^{n} \cdot \boldsymbol{n}_{\sigma, K}\right| H^{\prime \prime}\left(\bar{\varrho}_{\sigma}^{n}\right) \frac{\left(\varrho_{K}^{n}-\varrho_{L}^{n}\right)^{2}}{2}
$$

with some $\bar{\varrho}_{\sigma}^{n} \in\left[\min \left(\varrho_{K}^{n}, \varrho_{L}^{n}\right), \max \left(\varrho_{K}^{n}, \varrho_{L}^{n}\right)\right]$.

Step 4: Conclusion

Collecting the results of Steps 1-3 we arrive at

$$
\begin{aligned}
& \sum_{K \in \mathcal{T}} \frac{1}{2} \frac{|K|}{k}\left(\varrho_{K}^{n}\left|\boldsymbol{u}_{K}^{n}\right|^{2}-\varrho_{K}^{n-1}\left|\boldsymbol{u}_{K}^{n-1}\right|^{2}\right)+\sum_{K \in \mathcal{T}} \frac{|K|}{k}\left(H\left(\varrho_{K}^{n}\right)-H\left(\varrho_{K}^{n-1}\right)\right)+\sum_{K \in \mathcal{T}}\left(\mu \int_{K}\left|\nabla_{x} \boldsymbol{u}^{n}\right|^{2} \mathrm{~d} x\right. \\
&\left.+(\mu+\lambda) \int_{K}\left|\operatorname{div} \boldsymbol{u}^{n}\right|^{2} \mathrm{~d} x\right)+\sum_{K \in \mathcal{T}} \frac{|K|}{k} \varrho_{K}^{n-1} \frac{\left|\boldsymbol{u}_{K}^{n}-\boldsymbol{u}_{K}^{n-1}\right|^{2}}{2}+\sum_{K \in \mathcal{T}} \frac{|K|}{k} H^{\prime \prime}\left(\varrho_{K}^{n-1, n}\right) \frac{\left|\varrho_{K}^{n}-\varrho_{K}^{n-1}\right|^{2}}{2} \\
& \quad+\sum_{\substack{\sigma \in \mathcal{E}_{\text {int }} \\
\sigma=K \mid L}}|\sigma| \varrho_{\sigma}^{n, \text { up }} \frac{\left(\boldsymbol{u}_{K}^{n}-\boldsymbol{u}_{L}^{n}\right)^{2}}{2}\left|\boldsymbol{u}_{\sigma}^{n} \cdot \boldsymbol{n}_{\sigma, K}\right|+\sum_{\substack{\sigma \in \mathcal{E}_{\text {int }} \\
\sigma=K \mid L}}|\sigma| H^{\prime \prime}\left(\varrho_{\sigma}^{n}\right) \frac{\left(\varrho_{K}^{n}-\varrho_{L}^{n}\right)^{2}}{2}\left|\boldsymbol{u}_{\sigma}^{n} \cdot \mathbf{n}_{\sigma, K}\right|=0
\end{aligned}
$$

At this stage, we get the statement of Lemma 4.1 by multiplying (4.8) $)^{n}$ by $k$ and summing from $n=1$ to $n=m$. Lemma 4.1 is proved. 


\subsection{Estimates}

We have the following corollary of Lemma 4.1.

Corollary 4.1. (1) Under assumptions of Lemma [4.1, there exists $c=c\left(M_{0}, E_{0}\right)>0$ (independent of $h$ and $k$ ) such that

$$
\begin{gathered}
|\boldsymbol{u}|_{L^{2}\left(0, T ; V_{h}^{2}\left(\Omega ; \mathbb{R}^{3}\right)\right.} \leq c \\
\|\boldsymbol{u}\|_{L^{2}\left(0, T ; L^{6}\left(\Omega ; \mathbb{R}^{3}\right)\right)} \leq c \\
\left\|\varrho \hat{\boldsymbol{u}}^{2}\right\|_{L^{\infty}\left(0, T ; L^{1}(\Omega)\right)} \leq c .
\end{gathered}
$$

(2) If in addition the pressure satisfies assumption (1.6) then

$$
\|\varrho\|_{L^{\infty}\left(0, T ; L^{\gamma}(\Omega)\right)} \leq c
$$

(3) If the pair $(r, \boldsymbol{U})$ belongs to the class (3.18) there exists $c=c\left(M_{0}, E_{0}, \underline{r}, \bar{r},\|\boldsymbol{U}, \nabla \boldsymbol{U}\|_{L^{\infty}\left(Q_{T} ; \mathbb{R}^{12}\right)}\right)>0$ such that for all $n=1, \ldots, N$,

$$
\mathcal{E}\left(\varrho^{n}, \boldsymbol{u}^{n} \mid r^{n}, \boldsymbol{U}^{n}\right) \leq c,
$$

where the discrete relative energy $\mathcal{E}$ is defined in (3.16).

Proof. Recall that

$$
|\boldsymbol{u}|_{L^{2}\left(0, T ; V_{h}^{2}\left(\Omega ; \mathbb{R}^{3}\right)\right.}^{2}=k \sum_{n=1}^{N} \sum_{K \in \mathcal{T}} \int_{K}\left|\nabla_{x} \boldsymbol{u}^{n}\right|^{2} \mathrm{~d} x ;
$$

the estimate (4.9) follows from (4.1). The estimate (4.10) holds due to imbedding (9.30) in Lemma 9.3 and bound (4.9). The estimate (4.11) is just a short transcription of the bound for the kinetic energy in (4.1).

We prove estimate (4.12). First, we deduce from (1.5) and the definition (2.4) of $H$ that $0 \leq-H(\varrho) \leq$ $c_{1}$ with some $c_{1}>0$, provided $0<\varrho \leq 1$ and $H(\varrho)>0$ if $\varrho>1$. This fact in combination with the bound for $\int_{\Omega} H(\varrho) \mathrm{d} x$ derived in (4.1) yields

$$
\int_{\Omega}|H(\varrho)| \mathrm{d} x \leq c<\infty
$$

Second, relations (1.5 1.7) imply that there are $\bar{\varrho}>1$ and $0<\underline{p}<\bar{p}<\infty$ such that

$$
\left\{\begin{array}{c}
\varrho^{\alpha} p_{0} / 2 \leq \frac{p(\varrho)}{\varrho^{2}} \text { if } 0<\varrho<1 / \bar{\varrho} \\
\underline{p} \leq \frac{p(\varrho)}{\varrho^{2}} \leq \bar{p} \text { if } 1 / \bar{\varrho} \leq \varrho \leq \bar{\varrho}, \\
\varrho^{\gamma-2} p_{\infty} / 2 \leq \frac{p(\varrho)}{\varrho^{2}} \text { if } \varrho>2 \bar{\varrho}
\end{array}\right\} .
$$

Using these bounds and the definition (2.4) of $H$ we verify that

$$
\varrho^{\gamma} \leq c(|H(\varrho)|+\varrho+1)
$$

with a convenient positive constant $c$. Now, bound (4.12) follows readily from the boundedness of $\int_{\Omega} \varrho^{m} \mathrm{~d} x \equiv \sum_{K \in \mathcal{T}}|K| \varrho_{K}^{m}$ and $\int_{\Omega} H\left(\varrho^{m}\right) \mathrm{d} x \equiv \sum_{K \in \mathcal{T}}|K| H\left(\varrho_{K}^{m}\right)$ established in (3.15) and (4.1).

Finally, to get (4.13), we have employed (2.5), (3.16), (3.15), (4.14) to estimate $\int_{\Omega} E\left(\varrho^{n} \mid \hat{r}^{n}\right) \mathrm{d} x$ and (4.11), (9.3), (9.22) to evaluate $\sum_{K \in \mathcal{T}} \int_{K} \varrho_{K}^{n}\left|\boldsymbol{U}_{h, K}^{n}-\boldsymbol{u}_{K}^{n}\right|^{2} \mathrm{~d} x$.

The following estimates are obtained thanks to the numerical diffusion due to the upwinding, as is classical in the framework of hyperbolic conservation laws, see e.g. [9]. 
Lemma 4.2 (Dissipation estimates on the density). Let $\left(\varrho^{0}, \boldsymbol{u}^{0}\right) \in L_{h}^{+}(\Omega) \times \boldsymbol{W}_{h}(\Omega)$. Suppose that $\left(\varrho^{n}\right)_{1 \leq n \leq N} \subset\left[L_{h}^{+}(\Omega)\right]^{N},\left(\boldsymbol{u}^{n}\right)_{1 \leq n \leq N} \subset\left[\boldsymbol{W}_{h}\right]^{N}(\Omega)$ is a solution of problem (3.14). Finally assume that the pressure satisfies hypotheses (1.5) and (1.6). Then we have:

(1) If $\gamma \geq 2$ then there exists $c=c\left(\gamma, \theta_{0}, E_{0}\right)>0$ such that

$$
k \sum_{n=1}^{N} \sum_{\sigma=K \mid L \in \mathcal{E}_{\text {int }}}|\sigma| \frac{\left(\varrho_{K}^{n}-\varrho_{L}^{n}\right)^{2}}{\max \left(\varrho_{K}^{n}, \varrho_{L}^{n}\right)}\left|\boldsymbol{u}_{\sigma}^{n} \cdot \mathbf{n}_{\sigma, K}\right| \leq c .
$$

(2) If $\gamma \in[1,2)$ and the pressure satisfies additionally assumption (1.7) then there exists $c=c\left(M_{0}, E_{0}\right)>$ 0 such that

$$
\begin{aligned}
& k \sum_{n=1}^{N} \sum_{\sigma=K \mid L \in \mathcal{E}_{\text {int }}}|\sigma| \frac{\left(\varrho_{K}^{n}-\varrho_{L}^{n}\right)^{2}}{\left[\max \left(\varrho_{K}^{n}, \varrho_{L}^{n}\right)\right]^{2-\gamma}} 1_{\left\{\varrho_{\sigma}^{n} \geq 1\right\}}\left|\boldsymbol{u}_{\sigma}^{n} \cdot \mathbf{n}_{\sigma, K}\right| \\
& \quad+k \sum_{n=1}^{N} \sum_{\sigma=K \mid L \in \mathcal{E}_{\text {int }}}|\sigma|\left(\varrho_{K}^{n}-\varrho_{L}^{n}\right)^{2} 1_{\left\{\varrho_{\sigma}^{n}<1\right\}}\left|\boldsymbol{u}_{\sigma}^{n} \cdot \mathbf{n}_{\sigma, K}\right| \leq c,
\end{aligned}
$$

where the numbers $\bar{\varrho}_{\sigma}^{n}$ are defined in Lemma 4.1 .

Proof. We start by proving the simpler statement (2). Taking into account the continuity of the pressure, we deduce from assumptions (1.6) and (1.7) that there exist numbers $\bar{p}_{0}>0, \bar{p}_{\infty}>0$ such that

$$
H^{\prime \prime}(s) \geq\left\{\begin{array}{l}
\frac{\bar{p}_{\infty}}{s^{2-\gamma},} \quad \text { if } s \geq 1, \\
\bar{p}_{0} s^{\alpha} \geq \bar{p}_{0}, \quad \text { if } s<1, .
\end{array}\right.
$$

whence, splitting the sum in the definition of the term $\left[D_{\text {space }}^{N, \Delta \varrho}\right]$ (see (4.2di) $)$ into two sums, where $(\sigma, n)$ satisfies $\bar{\varrho}_{\sigma}^{n} \geq 1$ for the first one and $\varrho_{\sigma}^{n}<1$ for the second, we obtain the desired result.

Let us now turn to the proof of statement (1). Multiplying the discrete continuity equation $(3.14 \mathrm{a})_{K}^{n}$ by $\ln \varrho_{K}^{n}$ and summing over $K \in \mathcal{T}$, we get

$$
\sum_{K \in \mathcal{T}}|K| \frac{\varrho_{K}^{n}-\varrho_{K}^{n-1}}{k} \ln \varrho_{K}^{n}+\sum_{K \in \mathcal{T}} \sum_{\sigma \in \mathcal{E}(K), \sigma=K \mid L}\left(\ln \varrho_{K}^{n}\right) \varrho_{\sigma}^{n, \text { up }} \boldsymbol{u}_{\sigma}^{n} \cdot \boldsymbol{n}_{\sigma, K}=0 .
$$

By virtue of the convexity of the function $\varrho \mapsto \varrho \ln \varrho-\varrho$ on the positive real line, and due to the Taylor formula, we have

$$
\varrho_{K}^{n} \ln \varrho_{K}^{n}-\varrho_{K}^{n-1} \ln \varrho_{K}^{n-1}-\left(\varrho_{K}^{n}-\varrho_{K}^{n-1}\right) \leq \ln \varrho_{K}^{n}\left(\varrho_{K}^{n}-\varrho_{K}^{n-1}\right) ;
$$

whence, thanks to the mass conservation (3.15) and the definition of $\varrho_{\sigma}^{\text {up }}$, we arrive at

$$
\begin{aligned}
\sum_{K \in \mathcal{T}}|K| \frac{\varrho_{K}^{n} \ln \varrho_{K}^{n}-\varrho_{K}^{n-1} \ln \varrho_{K}^{n-1}}{k}+\sum_{\substack{\sigma \in \mathcal{E}_{\text {int }} \\
\sigma=K \mid L}}|\sigma| \varrho_{K}^{n}\left[\boldsymbol{u}_{\sigma}^{n} \cdot \boldsymbol{n}_{\sigma, K}\right]^{+}\left(\ln \varrho_{K}^{n}-\ln \varrho_{L}^{n}\right) \\
+\sum_{\substack{\sigma \in \mathcal{E}_{\text {int }} \\
\sigma=K \mid L}}|\sigma| \varrho_{L}^{n}\left[\boldsymbol{u}_{\sigma}^{n} \cdot \boldsymbol{n}_{\sigma, L}\right]^{+}\left(\ln \varrho_{L}^{n}-\ln \varrho_{K}^{n}\right) \leq 0
\end{aligned}
$$

or equivalently

$$
\begin{aligned}
& k \sum_{\substack{\sigma \in \mathcal{E}_{\text {int }} \\
\sigma=K \mid L}}|\sigma|\left[\boldsymbol{u}_{\sigma}^{n} \cdot \boldsymbol{n}_{\sigma, K}\right]^{+}\left(\varrho_{K}^{n}\left(\ln \varrho_{K}^{n}-\ln \varrho_{L}^{n}\right)-\left(\varrho_{K}^{n}-\varrho_{L}^{n}\right)\right)+k \sum_{\substack{\sigma \in \mathcal{E}_{\text {int }} \\
\sigma=K \mid L}}|\sigma|\left[\boldsymbol{u}_{\sigma}^{n} \cdot \boldsymbol{n}_{\sigma, L}\right]^{+}\left(\varrho_{L}^{n}\left(\ln \varrho_{L}^{n}-\ln \varrho_{K}^{n}\right)-\left(\varrho_{L}^{n}-\varrho_{K}^{n}\right)\right) \leq \\
- & \left.\left.\sum_{K \in \mathcal{T}}|K|\left(\varrho_{K}^{n} \ln \varrho_{K}^{n}-\varrho_{K}^{n-1} \ln \varrho_{K}^{n-1}\right)+k \sum_{\substack{\sigma \in \mathcal{E}_{\text {int }} \\
\sigma=K \mid L}}|\sigma|\left(\left[\boldsymbol{u}_{\sigma}^{n} \cdot \boldsymbol{n}_{\sigma, K}\right]^{+}\left(\varrho_{L}^{n}-\varrho_{K}^{n}\right)\right)+\left[\boldsymbol{u}_{\sigma}^{n} \cdot \boldsymbol{n}_{\sigma, L}\right]^{+}\left(\varrho_{K}^{n}-\varrho_{L}^{n}\right)\right)\right) .
\end{aligned}
$$


From [16, Lemma C.5], we know that if $\varphi$ and $\psi$ are functions in $C^{1}((0, \infty) ; \mathbb{R})$ such that $s \psi^{\prime}(s)=\varphi^{\prime}(s)$ for all $s \in(0, \infty)$, then for any $(a, b) \in(0, \infty)^{2}$ there exits $c \in[a, b]$ such that

$$
(\psi(b)-\psi(a)) b-(\varphi(b)-\varphi(a))=\frac{1}{2}(b-a)^{2} \psi^{\prime}(c) .
$$

Applying this result with $\psi(s)=\ln s, \varphi(s)=s$ we obtain that the left hand side of (4.17) is greater or equal to

$$
k \sum_{\substack{\sigma \in \mathcal{E}_{\text {int }} \sigma=K \mid L\\}}|\sigma|\left(\left[\boldsymbol{u}_{\sigma}^{n} \cdot \boldsymbol{n}_{\sigma, K}\right]^{+}+\left[\boldsymbol{u}_{\sigma}^{n} \cdot \boldsymbol{n}_{\sigma, L}\right]^{+}\right) \frac{\left(\varrho_{K}^{n}-\varrho_{L}^{n}\right)^{2}}{\max \left(\varrho_{K}^{n}, \varrho_{L}^{n}\right)} .
$$

On the other hand, the first term at the right hand side is bounded from above by $\left\|\varrho^{n}\right\|_{L^{\gamma}(\Omega)}^{\gamma}$. Finally the second term at the right hand side is equal to

$$
-k \sum_{K \in \mathcal{T}} \int_{K} \varrho_{K}^{n} \operatorname{div} \boldsymbol{u}^{n} \leq k \sum_{K \in \mathcal{T}}\left\|\varrho_{K}\right\|_{L^{2}(K)}\left\|\operatorname{div} \mathbf{u}^{n}\right\|_{L^{2}(K)}
$$

whence bounded from above by $k\left\|\boldsymbol{u}^{n}\right\|_{V_{h}^{2}\left(\Omega ; \mathbb{R}^{3}\right)}\left\|\varrho^{n}\right\|_{L^{2}(\Omega)}$, where we have used the Hölder inequality and the definition of the $V_{h}^{2}(\Omega)$-norm. The statement (1) of Lemma 4.2 now follows from the estimates of Corollary 4.1 .

\section{5 - Exact relative energy inequality for the discrete problem}

The goal of this section is to prove the discrete version of the relative energy inequality.

Theorem 5.1. Suppose that $\Omega \subset \mathbb{R}^{3}$ is a polyhedral domain and $\mathcal{T}$ its regular triangulation introduced in Section 3.1. Let p satisfy hypotheses (1.5) and the viscosity coefficient $\mu, \lambda$ obey (1.4). Let $\left(\varrho^{0}, \boldsymbol{u}^{0}\right) \in$ $L_{h}^{+}(\Omega) \times \boldsymbol{W}_{h}(\Omega)$ and suppose that $\left(\varrho^{n}\right)_{1 \leq n \leq N} \in\left[L_{h}^{+}(\Omega)\right]^{N},\left(\boldsymbol{u}^{n}\right)_{1 \leq n \leq N} \in\left[\boldsymbol{W}_{h}(\Omega)\right]^{N}$ is a solution of the discrete problem (3.14). Then there holds for all $m=1, \ldots, N$,

$$
\begin{aligned}
\sum_{K \in \mathcal{T}} \frac{1}{2} \mid & K\left|\left(\varrho_{K}^{m}\left|\boldsymbol{u}_{K}^{m}-\boldsymbol{U}_{h, K}^{m}\right|^{2}-\varrho_{K}^{0}\left|\boldsymbol{u}_{K}^{0}-\boldsymbol{U}_{h, K}^{0}\right|^{2}\right)+\sum_{K \in \mathcal{T}}\right| K \mid\left(E\left(\varrho_{K}^{m} \mid r_{K}^{m}\right)-E\left(\varrho_{K}^{0} \mid r_{K}^{0}\right)\right) \\
& +k \sum_{n=1}^{m} \sum_{K \in \mathcal{T}}\left(\mu \int_{K}\left|\nabla_{x}\left(\boldsymbol{u}^{n}-\boldsymbol{U}_{h}^{n}\right)\right|^{2} \mathrm{~d} x+(\mu+\lambda) \int_{K}\left|\operatorname{div}\left(\boldsymbol{u}^{n}-\boldsymbol{U}_{h}^{n}\right)\right|^{2} \mathrm{~d} x\right) \\
\leq & k \sum_{n=1}^{m} \sum_{K \in \mathcal{T}}\left(\mu \int_{K} \nabla_{x} \boldsymbol{U}_{h}^{n}: \nabla_{x}\left(\boldsymbol{U}_{h}^{n}-\boldsymbol{u}^{n}\right) \mathrm{d} x+(\mu+\lambda) \int_{K} \operatorname{div} \boldsymbol{U}_{h}^{n} \operatorname{div}\left(\boldsymbol{U}_{h}^{n}-\boldsymbol{u}^{n}\right) \mathrm{d} x\right) \\
& +k \sum_{n=1}^{m} \sum_{K \in \mathcal{T}}|K| \varrho_{K}^{n-1} \frac{\boldsymbol{U}_{h, K}^{n}-\boldsymbol{U}_{h, K}^{n-1}}{k} \cdot\left(\frac{\boldsymbol{U}_{h, K}^{n-1}+\boldsymbol{U}_{h, K}^{n}}{2}-\boldsymbol{u}_{K}^{n-1}\right) \\
& -k \sum_{n=1}^{m} \sum_{K \in \mathcal{T}} \sum_{\sigma \in \mathcal{E}(K)}|\sigma| \varrho_{\sigma}^{n, \mathrm{up}}\left(\frac{\boldsymbol{U}_{h, K}^{n}+\boldsymbol{U}_{h, L}^{n}}{2}-\hat{\boldsymbol{u}}_{\sigma}^{n, \mathrm{up}}\right) \cdot \boldsymbol{U}_{h, K}^{n}\left[\boldsymbol{u}_{\sigma}^{n} \cdot \boldsymbol{n}_{\sigma, K}\right] \\
& -k \sum_{n=1}^{m} \sum_{K \in \mathcal{T}} \sum_{\sigma \in \mathcal{E}(K)}|\sigma| p\left(\varrho_{K}^{n}\right)\left[\boldsymbol{U}_{h, \sigma}^{n} \cdot \boldsymbol{n}_{\sigma, K}\right] \\
& +k \sum_{\sigma=K \mid L}^{m} \sum_{K \in \mathcal{T}} \frac{|K|}{k}\left(r_{K}^{n}-\varrho_{K}^{n}\right)\left(H^{\prime}\left(r_{K}^{n}\right)-H^{\prime}\left(r_{K}^{n-1}\right)\right) \\
& +k \sum_{n=1}^{m} \sum_{K \in \mathcal{T}} \sum_{\sigma \in \mathcal{E}(K)}|\sigma| \varrho_{\sigma=K \mid L}^{n, \mathrm{up}} H^{\prime}\left(r_{K}^{n-1}\right)\left[\boldsymbol{u}_{\sigma}^{n} \cdot \boldsymbol{n}_{\sigma, K}\right]
\end{aligned}
$$


for any $0<r \in C^{1}([0, T] \times \bar{\Omega}), \boldsymbol{U} \in C^{1}([0, T] \times \bar{\Omega}),\left.\boldsymbol{U}\right|_{\partial \Omega}=0$, where we have used notation (3.17) for $r^{n}, \mathbf{U}^{n}$ and (3.73.8) for $\mathbf{U}_{h}^{n}, \mathbf{U}_{h, K}^{n}, r_{K}^{n}, \mathbf{u}_{\sigma}^{n}$.

We notice, comparing the terms in the "discrete" formula (5.1) with the terms in the "continuous" formula (2.7), that Theorem 5.1 represents a discrete counterpart of the "continuous" relative energy inequality (2.7). The rest of this section is devoted to its proof. To this end, we shall follow the proof of the "continuous" relative energy inequality (see [12] and [15]) and adapt it to the discrete case.

Proof. First, noting that the numerical diffusion represented by terms (4.2a 4.2d $)$ in the energy identity (4.1) is positive, we infer

$$
I_{1}+I_{2}+I_{3} \leq 0
$$

with

$$
\begin{aligned}
& I_{1}:=\sum_{K \in \mathcal{T}} \frac{1}{2} \frac{|K|}{k}\left(\varrho_{K}^{n}\left|\boldsymbol{u}_{K}^{n}\right|^{2}-\varrho_{K}^{n-1}\left|\boldsymbol{u}_{K}^{n-1}\right|^{2}\right), \quad I_{2}:=\sum_{K \in \mathcal{T}} \frac{|K|}{k}\left(H\left(\varrho_{K}^{n}\right)-H\left(\varrho_{K}^{n-1}\right)\right), \\
& I_{3}:=\sum_{K \in \mathcal{T}}\left(\mu \int_{K}\left|\nabla_{x} \boldsymbol{u}^{n}\right|^{2} \mathrm{~d} x+(\mu+\lambda) \int_{K}\left|\operatorname{div} \boldsymbol{u}^{n}\right|^{2} \mathrm{~d} x\right) .
\end{aligned}
$$

Next, we multiply the discrete continuity equation $\left({ }_{3.14 a}\right)_{K}^{n}$ by $\frac{1}{2}\left|\mathbf{U}_{h, K}^{n}\right|^{2}$ and sum over $K \in \mathcal{T}$ to obtain

$$
I_{4}:=\sum_{K \in \mathcal{T}} \frac{1}{2} \frac{|K|}{k}\left(\varrho_{K}^{n}-\varrho_{K}^{n-1}\right)\left|\boldsymbol{U}_{h, K}^{n}\right|^{2}=-\sum_{\substack { K \in \mathcal{T} \\
\begin{subarray}{c}{\sigma \in \mathcal{E}(K) \\
\sigma=K \mid L{ K \in \mathcal { T } \\
\begin{subarray} { c } { \sigma \in \mathcal { E } ( K ) \\
\sigma = K | L } }\end{subarray}} \frac{1}{2}|\sigma| \varrho_{\sigma}^{n, \mathrm{up}}\left[\boldsymbol{u}_{\sigma}^{n} \cdot \boldsymbol{n}_{\sigma, K}\right]\left|\boldsymbol{U}_{h, K}^{n}\right|^{2}:=J_{1}
$$

In the next step, taking $-\boldsymbol{U}^{n}$ as test function in the discrete momentum equation (3.14b); we get

$$
I_{5}=-\sum_{K \in \mathcal{T}} \frac{|K|}{k}\left(\varrho_{K}^{n} \boldsymbol{u}_{K}^{n}-\varrho_{K}^{n-1} \boldsymbol{u}_{K}^{n-1}\right) \cdot \boldsymbol{U}_{h, K}^{n}=J_{2}+J_{3}+J_{4}
$$

with

$$
\begin{aligned}
& J_{2}=\sum_{K \in \mathcal{T}} \sum_{\substack{\sigma \in \mathcal{E}(K) \\
\sigma=K \mid L}}|\sigma| \varrho_{\sigma}^{n, \text { up }} \hat{\boldsymbol{u}}_{\sigma}^{n, \text { up }} \cdot \boldsymbol{U}_{h, K}^{n}\left[\boldsymbol{u}_{\sigma}^{n} \cdot \mathbf{n}_{\sigma, K}\right], \\
& J_{3}=\mu \sum_{K \in \mathcal{T}} \int_{K} \nabla \boldsymbol{u}^{n}: \nabla \boldsymbol{U}_{h}^{n} \mathrm{~d} x+(\mu+\lambda) \sum_{K \in \mathcal{T}} \int_{K} \operatorname{div} \boldsymbol{u}^{n} \operatorname{div} \boldsymbol{U}_{h}^{n} \mathrm{~d} x
\end{aligned}
$$

and

$$
J_{4}=-\sum_{K \in \mathcal{T}} \sum_{\substack{\sigma \in \mathcal{E}(K) \\ \sigma=K \mid L}}|\sigma| p\left(\varrho_{K}^{n}\right)\left[\boldsymbol{U}_{\sigma}^{n} \cdot \mathbf{n}_{\sigma, K}\right]
$$

We then multiply the continuity equation $(3.14 \mathrm{a})_{K}^{n}$ by $H^{\prime}\left(r_{K}^{n-1}\right)$ and sum over all $K \in \mathcal{T}$ and obtain

$$
-\sum_{K \in \mathcal{T}} \frac{|K|}{k}\left(\varrho_{K}^{n}-\varrho_{K}^{n-1}\right) H^{\prime}\left(r_{K}^{n-1}\right)=\sum_{K \in \mathcal{T}} \sum_{\substack{\sigma \in \mathcal{E}(K) \\ \sigma=K \mid L}}|\sigma| \varrho_{\sigma}^{n, \text { up }}\left[\boldsymbol{u}_{\sigma}^{n} \cdot \boldsymbol{n}_{\sigma, K}\right] H^{\prime}\left(r_{K}^{n-1}\right) .
$$

Observing that $\varrho_{K}^{n} H^{\prime}\left(r_{K}^{n}\right)-\varrho_{K}^{n-1} H^{\prime}\left(r_{K}^{n-1}\right)=\varrho_{K}^{n}\left(H^{\prime}\left(r_{K}^{n}\right)-H^{\prime}\left(r_{K}^{n-1}\right)\right)+\left(\varrho_{K}^{n}-\varrho_{K}^{n-1}\right) H^{\prime}\left(r_{K}^{n-1}\right)$, we rewrite the last identity in the form

$$
\begin{aligned}
& I_{6}:=-\sum_{K \in \mathcal{T}} \frac{|K|}{k}\left(\varrho_{K}^{n} H^{\prime}\left(r_{K}^{n}\right)-\varrho_{K}^{n-1} H^{\prime}\left(r_{K}^{n-1}\right)\right)=J_{5}+J_{6} \\
& \text { with } J_{5}=-\sum_{K \in \mathcal{T}} \frac{|K|}{k} \varrho_{K}^{n}\left(H^{\prime}\left(r_{K}^{n}\right)-H^{\prime}\left(r_{K}^{n-1}\right)\right) \text { and } J_{6}=\sum_{K \in \mathcal{T}} \sum_{\substack{\sigma \in \mathcal{E}(K) \\
\sigma=K \mid L}}|\sigma| \varrho_{\sigma}^{n, \text { up }}\left[\boldsymbol{u}_{\sigma}^{n} \cdot \boldsymbol{n}_{\sigma, K}\right] H^{\prime}\left(r_{K}^{n-1}\right) \text {. }
\end{aligned}
$$


Finally, thanks to the convexity of the function $H$, we have

$$
\begin{aligned}
I_{7} & :=\sum_{K \in \mathcal{T}} \frac{|K|}{k}\left[\left(r_{K}^{n} H^{\prime}\left(r_{K}^{n}\right)-H\left(r_{K}^{n}\right)\right)-\left(r_{K}^{n-1} H^{\prime}\left(r_{K}^{n-1}\right)-H\left(r_{K}^{n-1}\right)\right)\right] \\
& =\sum_{K \in \mathcal{T}} \frac{|K|}{k} r_{K}^{n}\left(H^{\prime}\left(r_{K}^{n}\right)-H^{\prime}\left(r_{K}^{n-1}\right)\right)-\sum_{K \in \mathcal{T}} \frac{|K|}{k}\left(H\left(r_{K}^{n}\right)-\left(r_{K}^{n}-r_{K}^{n-1}\right) H^{\prime}\left(r_{K}^{n-1}\right)-H\left(r_{K}^{n-1}\right)\right. \\
& \leq \sum_{K \in \mathcal{T}} \frac{|K|}{k} r_{K}^{n}\left(H^{\prime}\left(r_{K}^{n}\right)-H^{\prime}\left(r_{K}^{n-1}\right)\right):=J_{7},
\end{aligned}
$$

Now, we gather the expressions (5.2)-(5.5); this is performed in several steps.

Step 1: Term $I_{1}+I_{4}+I_{5}$. We observe that

$$
\left\{\begin{array}{l}
\frac{\left|\boldsymbol{U}_{h, K}^{n}\right|^{2}}{2}\left(\varrho_{K}^{n}-\varrho_{K}^{n-1}\right)=\frac{\varrho_{K}^{n}\left|\boldsymbol{U}_{h, K}^{n}\right|^{2}-\varrho_{K}^{n-1}\left|\boldsymbol{U}_{h, K}^{n-1}\right|^{2}}{2}+\varrho_{K}^{n-1} \frac{\boldsymbol{U}_{h, K}^{n-1}+\boldsymbol{U}_{h, K}^{n}}{2} \cdot\left(\boldsymbol{U}_{h, K}^{n-1}-\boldsymbol{U}_{h, K}^{n}\right), \\
-\left(\varrho_{K}^{n} \boldsymbol{u}_{K}^{n}-\varrho_{K}^{n-1} \boldsymbol{u}_{K}^{n-1}\right) \cdot \boldsymbol{U}_{h, K}^{n}=-\left(\varrho_{K}^{n} \boldsymbol{u}_{K}^{n} \cdot \boldsymbol{U}_{h, K}^{n}-\varrho_{K}^{n-1} \boldsymbol{u}_{K}^{n-1} \cdot \boldsymbol{U}_{h, K}^{n-1}\right)-\varrho_{K}^{n-1} \boldsymbol{u}_{K}^{n-1} \cdot\left(\boldsymbol{U}_{h, K}^{n-1}-\boldsymbol{U}_{h, K}^{n}\right) .
\end{array}\right.
$$

Consequently,

$$
\begin{aligned}
I_{1}+I_{4}+I_{5}=\sum_{K \in \mathcal{T}} \frac{1}{2} \frac{|K|}{k}\left(\varrho_{K}^{n}\left|\boldsymbol{u}_{K}^{n}-\boldsymbol{U}_{h, K}^{n}\right|^{2}-\varrho_{K}^{n-1}\left|\boldsymbol{u}_{K}^{n-1}-\boldsymbol{U}_{h, K}^{n-1}\right|^{2}\right) & \\
& -\sum_{K \in \mathcal{T}}|K| \varrho_{K}^{n-1} \frac{\boldsymbol{U}_{h, K}^{n}-\boldsymbol{U}_{h, K}^{n-1}}{k} \cdot\left(\frac{\boldsymbol{U}_{h, K}^{n-1}+\boldsymbol{U}_{h, K}^{n}}{2}-\boldsymbol{u}_{K}^{n-1}\right)
\end{aligned}
$$

Step 2: Term $J_{1}+J_{2}$. The contribution of the face $\sigma=K \mid L$ to $J_{1}$ reads

$$
-|\sigma| \varrho_{K}^{n} \frac{\boldsymbol{U}_{h, K}^{n}+\boldsymbol{U}_{h, L}^{n}}{2} \cdot\left(\boldsymbol{U}_{h, K}^{n}-\boldsymbol{U}_{h, L}^{n}\right)\left[\boldsymbol{u}_{\sigma}^{n} \cdot \boldsymbol{n}_{\sigma, K}\right]^{+}-|\sigma| \varrho_{L}^{n} \frac{\boldsymbol{U}_{h, K}^{n}+\boldsymbol{U}_{h, L}^{n}}{2} \cdot\left(\boldsymbol{U}_{h, L}^{n}-\boldsymbol{U}_{h, K}^{n}\right)\left[\boldsymbol{u}_{\sigma}^{n} \cdot \boldsymbol{n}_{\sigma, L}\right]^{+} .
$$

Similarly, the contribution of the face $\sigma=K \mid L$ to $J_{2}$ is

$$
|\sigma| \varrho_{K}^{n} \boldsymbol{u}_{K}^{n} \cdot\left(\boldsymbol{U}_{h, K}^{n}-\boldsymbol{U}_{h, L}^{n}\right)\left[\boldsymbol{u}_{\sigma}^{n} \cdot \boldsymbol{n}_{\sigma, K}\right]^{+}+|\sigma| \varrho_{L}^{n} \boldsymbol{u}_{L}^{n} \cdot\left(\boldsymbol{U}_{h, L}^{n}-\boldsymbol{U}_{h, K}^{n}\right)\left[\boldsymbol{u}_{\sigma}^{n} \cdot \boldsymbol{n}_{\sigma, L}\right]^{+} .
$$

Consequently,

$$
J_{1}+J_{2}=-\sum_{K \in \mathcal{T}} \sum_{\sigma=K \mid L \in \mathcal{E}_{K}}|\sigma| \varrho_{\sigma}^{n, \mathrm{up}}\left(\frac{\boldsymbol{U}_{h, K}^{n}+\boldsymbol{U}_{h, L}^{n}}{2}-\hat{\boldsymbol{u}}_{\sigma}^{n, \mathrm{up}}\right) \cdot \boldsymbol{U}_{h, K}^{n}\left[\boldsymbol{u}_{\sigma}^{n} \cdot \boldsymbol{n}_{\sigma, K}\right] .
$$

Step 3: Term $I_{3}-J_{3}$. This term can be written in the form

$$
\begin{aligned}
I_{3}-J_{3}= & \sum_{K \in \mathcal{T}}\left(\mu \int_{K}\left|\nabla_{x}\left(\boldsymbol{u}^{n}-\boldsymbol{U}_{h}^{n}\right)\right|^{2} \mathrm{~d} x+(\mu+\lambda) \int_{K}\left|\operatorname{div}\left(\boldsymbol{u}^{n}-\boldsymbol{U}_{h}^{n}\right)\right|^{2} \mathrm{~d} x\right) \\
& -\sum_{K \in \mathcal{T}} \mu \int_{K}\left(\nabla \boldsymbol{U}_{h}^{n}: \nabla\left(\boldsymbol{U}_{h}^{n}-\boldsymbol{u}^{n}\right)+(\mu+\lambda) \int_{K} \operatorname{div} \boldsymbol{U}_{h}^{n} \operatorname{div}\left(\boldsymbol{U}_{h}^{n}-\boldsymbol{u}^{n}\right)\right) .
\end{aligned}
$$

Step 4: Term $I_{2}+I_{6}+I_{7}$. By virtue of (5.2), (5.4 5.5), we easily find that

$$
I_{2}+I_{6}+I_{7}=\sum_{K \in \mathcal{T}} \frac{|K|}{k}\left(E\left(\varrho_{K}^{n} \mid r_{K}^{n}\right)-E\left(\varrho_{K}^{n-1} \mid r_{K}^{n-1}\right)\right)
$$

where the function $E$ is defined in (2.5). 
Step 5: Term $J_{5}+J_{6}+J_{7}$. Coming back to (5.4 5.5), we deduce that

$$
J_{5}+J_{6}+J_{7}=\sum_{K \in \mathcal{T}} \frac{|K|}{k}\left(r_{K}^{n}-\varrho_{K}^{n}\right)\left(H^{\prime}\left(r_{K}^{n}\right)-H^{\prime}\left(r_{K}^{n-1}\right)\right)+\sum_{K \in \mathcal{T}} \sum_{\substack{\sigma \in \mathcal{E}(K) \\ \sigma=K \mid L}}|\sigma| \varrho_{\sigma}^{n, \mathrm{up}}\left[\boldsymbol{u}_{\sigma}^{n} \cdot \boldsymbol{n}_{\sigma, K}\right] H^{\prime}\left(r_{K}^{n-1}\right)
$$

Step 6: Conclusion

According to (5.2)-(5.5), we have

$$
\sum_{i=1}^{7} I_{i} \leq \sum_{i=1}^{7} J_{i}
$$

whence, writing this inequality by using expressions (5.6)-(5.10) calculated in steps 1-5, we get

$$
\begin{aligned}
\sum_{K \in \mathcal{T}} \frac{1}{2} \frac{|K|}{k} & \left(\varrho_{K}^{n}\left|\boldsymbol{u}_{K}^{n}-\boldsymbol{U}_{h, K}^{n}\right|^{2}-\varrho_{K}^{n-1}\left|\boldsymbol{u}_{K}^{n-1}-\boldsymbol{U}_{h, K}^{n-1}\right|^{2}\right)+\sum_{K \in \mathcal{T}} \frac{|K|}{k}\left(E\left(\varrho_{K}^{n} \mid r_{K}^{n}\right)-E\left(\varrho_{K}^{n-1} \mid r_{K}^{n-1}\right)\right) \\
& +\sum_{K \in \mathcal{T}}\left(\mu \int_{K}\left|\nabla_{x}\left(\boldsymbol{u}^{n}-\boldsymbol{U}_{h}^{n}\right)\right|^{2} \mathrm{~d} x+(\mu+\lambda) \int_{K}\left|\operatorname{div}\left(\boldsymbol{u}^{n}-\boldsymbol{U}_{h}^{n}\right)\right|^{2} \mathrm{~d} x\right) \\
\leq & \sum_{K \in \mathcal{T}}\left(\mu \int_{K} \nabla_{x} \boldsymbol{U}_{h}^{n}: \nabla_{x}\left(\boldsymbol{U}_{h}^{n}-\boldsymbol{u}^{n}\right) \mathrm{d} x+(\mu+\lambda) \int_{K} \operatorname{div} \boldsymbol{U}_{h}^{n} \operatorname{div}\left(\boldsymbol{U}_{h}^{n}-\boldsymbol{u}^{n}\right) \mathrm{d} x\right) \\
& +\sum_{K \in \mathcal{T}}|K| \varrho_{K}^{n-1} \frac{\boldsymbol{U}_{h, K}^{n}-\boldsymbol{U}_{h, K}^{n-1}}{k} \cdot\left(\frac{\boldsymbol{U}_{h, K}^{n-1}+\boldsymbol{U}_{h, K}^{n}}{2}-\boldsymbol{u}_{K}^{n-1}\right) \\
& -\sum_{K \in \mathcal{T}} \sum_{\sigma=K \mid L \in \mathcal{E}_{K}}|\sigma| \varrho_{\sigma}^{n, \mathrm{up}}\left(\frac{\boldsymbol{U}_{h, K}^{n}+\boldsymbol{U}_{h, L}^{n}}{2}-\hat{\boldsymbol{u}}_{\sigma}^{n, \mathrm{up}}\right) \cdot \boldsymbol{U}_{h, K}^{n}\left[\boldsymbol{u}_{\sigma}^{n} \cdot \boldsymbol{n}_{\sigma, K}\right] \\
& -\sum_{K \in \mathcal{T}} \sum_{\sigma=K \mid L \in \mathcal{E}_{K}}|\sigma| p\left(\varrho_{K}^{n}\right)\left[\boldsymbol{U}_{h, \sigma}^{n} \cdot \boldsymbol{n}_{\sigma, K}\right]+\sum_{K \in \mathcal{T}} \frac{|K|}{k}\left(r_{K}^{n}-\varrho_{K}^{n}\right)\left(H^{\prime}\left(r_{K}^{n}\right)-H^{\prime}\left(r_{K}^{n-1}\right)\right) \\
& +\sum_{K \in \mathcal{T}} \sum_{\sigma=K \mid L \in \mathcal{E}_{K}}|\sigma| \varrho_{\sigma}^{n, \mathrm{up}} H^{\prime}\left(r_{K}^{n-1}\right)\left[\boldsymbol{u}_{\sigma}^{n} \cdot \boldsymbol{n}_{\sigma, K}\right] .
\end{aligned}
$$

We obtain formula (5.1) by summing $(5.11)^{n}$ from $n=1$ to $n=m$ and multiplying the resulting inequality by $k$.

\section{6 - Approximate relative energy inequality for the discrete problem}

The exact relative energy inequality as stated in Section 5 is a general inequality for the given numerical scheme, however it does not immediately provide a comparison of the approximate solution with the strong solution of the compressible Navier-Stokes equations. Its right hand side has to be conveniently transformed (modulo the possible appearance of residual terms vanishing as the space and time steps tend to 0) to provide such comparison tool via a Gronwall type argument.

The goal of this section is to derive a version of the discrete relative energy inequality, still with arbitrary (sufficiently regular) test functions $(r, \boldsymbol{U})$, that will be convenient for the comparison of the discrete solution with the strong solution.

Lemma 6.1 (Approximate relative energy inequality). Suppose that $\Omega \subset \mathbb{R}^{3}$ is a bounded polyhedral domain and $\mathcal{T}$ its regular triangulation introduced in Section [3.1. Let the pressure $p$ be a $C^{2}(0, \infty)$ function satisfying hypotheses (1.5), (1.6) with $\gamma \geq 3 / 2$ and satisfying the additional condition (1.7) if $\gamma<2$.

Let $\left(\varrho^{0}, \boldsymbol{u}^{0}\right) \in L_{h}^{+}(\Omega) \times \boldsymbol{W}_{h}(\Omega)$ and suppose that $\left(\varrho^{n}\right)_{1 \leq n \leq N} \in\left[L_{h}^{+}(\Omega)\right]^{N},\left(\boldsymbol{u}^{n}\right)_{1 \leq n \leq N} \in\left[\boldsymbol{W}_{h}(\Omega)\right]^{N}$ is a solution of the discrete problem (3.14) with the viscosity coefficients $\mu, \lambda$ obeying (1.4). 
Then there exists

$$
c=c\left(M_{0}, E_{0}, \underline{r}, \bar{r},\left|p^{\prime}\right|_{C^{1}[\underline{r}, \bar{r}]},\left\|\left(\partial_{t} r, \partial_{t}^{2} r, \nabla r, \partial_{t} \nabla r, \boldsymbol{U}, \partial_{t} \boldsymbol{U}, \nabla \boldsymbol{U}, \partial_{t} \nabla \boldsymbol{U}\right)\right\|_{L^{\infty}\left(Q_{T} ; \mathbb{R}^{31}\right)}\right)>0
$$

(where $\left.\bar{r}=\max _{(t, x) \in \overline{Q_{T}}} r(t, x), \underline{r}=\min _{(t, x) \in \overline{Q_{T}}} r(t, x)\right)$, such that for all $m=1, \ldots, N$, we have:

$$
\begin{aligned}
\mathcal{E}\left(\varrho^{m}, \boldsymbol{u}^{m} \mid r^{m}, \boldsymbol{U}^{m}\right)-\mathcal{E}\left(\varrho^{0}, \boldsymbol{u}^{0} \mid r(0), \boldsymbol{U}(0)\right) & \\
& \quad+k \sum_{n=1}^{m} \sum_{K \in \mathcal{T}}\left(\mu \int_{K}\left|\nabla_{x}\left(\boldsymbol{u}^{n}-\boldsymbol{U}_{h}^{n}\right)\right|^{2} \mathrm{~d} x+(\mu+\lambda) \int_{K}\left|\operatorname{div}\left(\boldsymbol{u}^{n}-\boldsymbol{U}_{h}^{n}\right)\right|^{2} \mathrm{~d} x\right) \\
\leq & k \sum_{n=1}^{m} \sum_{K \in \mathcal{T}}\left(\mu \int_{K} \nabla_{x} \boldsymbol{U}_{h}^{n}: \nabla_{x}\left(\boldsymbol{U}_{h}^{n}-\boldsymbol{u}^{n}\right) \mathrm{d} x+(\mu+\lambda) \int_{K} \operatorname{div} \boldsymbol{U}_{h}^{n} \operatorname{div}\left(\boldsymbol{U}_{h}^{n}-\boldsymbol{u}^{n}\right) \mathrm{d} x\right) \\
& +k \sum_{n=1}^{m} \sum_{K \in \mathcal{T}}|K| \varrho_{K}^{n-1} \frac{\boldsymbol{U}_{h, K}^{n}-\boldsymbol{U}_{h, K}^{n-1}}{k} \cdot\left(\boldsymbol{U}_{h, K}^{n}-\boldsymbol{u}_{K}^{n}\right) \\
+ & k \sum_{n=1}^{m} \sum_{K \in \mathcal{T}} \sum_{\sigma \in \mathcal{E}(K)}|\sigma| \varrho_{\sigma}^{n, \text { up }}\left(\hat{\boldsymbol{U}}_{h, \sigma}^{n, \text { up }}-\hat{\boldsymbol{u}}_{\sigma}^{n, \mathrm{up}}\right) \cdot\left(\boldsymbol{U}_{\sigma}^{n}-\boldsymbol{U}_{h, K}^{n}\right) \hat{\boldsymbol{U}}_{h, \sigma}^{n, \mathrm{up}} \cdot \boldsymbol{n}_{\sigma, K} \\
& -k \sum_{n=1}^{m} \sum_{K \in \mathcal{T}} \int_{K} p\left(\varrho_{K}^{n}\right) \operatorname{div} \boldsymbol{U}^{n} \mathrm{~d} x+k \sum_{n=1}^{m} \sum_{K \in \mathcal{T}} \int_{K}\left(r_{K}^{n}-\varrho_{K}^{n}\right) \frac{p^{\prime}\left(r_{K}^{n}\right)}{r_{K}^{n}}\left[\partial_{t} r\right]^{n} \mathrm{~d} x \\
& -k \sum_{n=1}^{m} \sum_{K \in \mathcal{T}} \int_{K} \frac{\varrho_{K}^{n}}{r_{K}^{n}} p^{\prime}\left(r_{K}^{n}\right) \boldsymbol{u}^{n} \cdot \nabla r^{n} \mathrm{~d} x+R_{h, k}^{m}+G^{m}
\end{aligned}
$$

for any pair $(r, \boldsymbol{U})$ belonging to the class (3.18), where

$$
\left|G^{m}\right| \leq c k \sum_{n=1}^{m} \mathcal{E}\left(\varrho^{n}, \boldsymbol{u}^{n} \mid r^{n}, U^{n}\right),\left|R_{h, k}^{m}\right| \leq c\left(\sqrt{k}+h^{A}\right), \quad \text { and } A=\left\{\begin{array}{c}
\frac{2 \gamma-3}{\gamma} \text { if } \gamma \in[3 / 2,2) \\
1 / 2 \text { if } \gamma \geq 2,
\end{array}\right.
$$

and where we have used notation (3.17) for $r^{n}, \mathbf{U}^{n}$ and (3.7 3.8) for $\mathbf{U}_{h}^{n}, \mathbf{U}_{h, K}^{n}, r_{K}^{n}, \mathbf{u}_{\sigma}^{n}$.

Proof. The right hand side of the relative energy inequality (5.1) is a sum $\sum_{i=1}^{6} T_{i}$, where

$$
\begin{aligned}
T_{1} & =k \sum_{n=1}^{m} \sum_{K \in \mathcal{T}}\left(\mu \int_{K} \nabla_{x} \boldsymbol{U}_{h}^{n}: \nabla_{x}\left(\boldsymbol{U}_{h}^{n}-\boldsymbol{u}^{n}\right) \mathrm{d} x+(\mu+\lambda) \int_{K} \operatorname{div} \boldsymbol{U}_{h}^{n} \operatorname{div}\left(\boldsymbol{U}_{h}^{n}-\boldsymbol{u}^{n}\right) \mathrm{d} x\right), \\
T_{2} & =k \sum_{n=1}^{m} \sum_{K \in \mathcal{T}}|K| \varrho_{K}^{n-1} \frac{\boldsymbol{U}_{h, K}^{n}-\boldsymbol{U}_{h, K}^{n-1}}{k} \cdot\left(\frac{\boldsymbol{U}_{h, K}^{n-1}+\boldsymbol{U}_{h, K}^{n}}{2}-\boldsymbol{u}_{K}^{n-1}\right), \\
T_{3} & =-k \sum_{n=1}^{m} \sum_{K \in \mathcal{T}} \sum_{\sigma=K \mid L \in \mathcal{E}(K)}|\sigma| \varrho_{\sigma}^{n, \mathrm{up}}\left(\frac{\boldsymbol{U}_{h, K}^{n}+\boldsymbol{U}_{h, L}^{n}}{2}-\hat{\boldsymbol{u}}_{\sigma}^{n, \mathrm{up}}\right) \cdot \boldsymbol{U}_{h, K}^{n}\left[\boldsymbol{u}_{\sigma}^{n} \cdot \boldsymbol{n}_{\sigma, K}\right], \\
T_{4}= & -k \sum_{n=1}^{m} \sum_{K \in \mathcal{T}} \sum_{\sigma=K \mid L \in \mathcal{E}(K)}|\sigma| p\left(\varrho_{K}\right)\left[\boldsymbol{U}_{h, \sigma}^{n} \cdot \boldsymbol{n}_{\sigma, K}\right], \\
T_{5}= & k \sum_{n=1}^{m} \sum_{K \in \mathcal{T}}|K|\left(r_{K}^{n}-\varrho_{K}^{n}\right) \frac{H^{\prime}\left(r_{K}^{n}\right)-H^{\prime}\left(r_{K}^{n-1}\right)}{k}, \\
T_{6}= & k \sum_{n=1}^{m} \sum_{K \in \mathcal{T}} \sum_{\sigma=K \mid L \in \mathcal{E}(K)}|\sigma| \varrho_{\sigma}^{n, \mathrm{up}} H^{\prime}\left(r_{K}^{n-1}\right)\left[\boldsymbol{u}_{\sigma}^{n} \cdot \boldsymbol{n}_{\sigma, K}\right] .
\end{aligned}
$$

The term $T_{1}$ will be kept as it is; all the other terms $T_{i}$ will be transformed to a more convenient form, as described in the following steps. 
Step 1: Term $T_{2}$. We have

$T_{2}=T_{2,1}+R_{2,1}$, with $T_{2,1}=k \sum_{n=1}^{m} \sum_{K \in \mathcal{T}}|K| \varrho_{K}^{n-1} \frac{\boldsymbol{U}_{h, K}^{n}-\boldsymbol{U}_{h, K}^{n-1}}{k} \cdot\left(\boldsymbol{U}_{h, K}^{n-1}-\boldsymbol{u}_{K}^{n-1}\right)$, and $R_{2,1}=k \sum_{n=1}^{m} \sum_{K \in \mathcal{T}} R_{2,1}^{n, K}$,

where

$$
R_{2,1}^{n, K}=\frac{|K|}{2} \varrho_{K}^{n-1} \frac{\left(\boldsymbol{U}_{h, K}^{n}-\boldsymbol{U}_{h, K}^{n-1}\right)^{2}}{k}=\frac{|K|}{2} \varrho_{K}^{n-1} \frac{\left(\left[\boldsymbol{U}^{n}-\boldsymbol{U}^{n-1}\right]_{h, K}\right)^{2}}{k} .
$$

We may write by virtue of the first order Taylor formula applied to function $t \mapsto \mathbf{U}(t, x)$,

$$
\begin{gathered}
\left|\frac{\left[\boldsymbol{U}^{n}-\boldsymbol{U}^{n-1}\right]_{h, K}}{k}\right|=\left|\frac{1}{|K|} \int_{K}\left[\frac{1}{k}\left[\int_{t_{n-1}}^{t_{n}} \partial_{t} \mathbf{U}(z, x) \mathrm{d} z\right]_{h}\right] \mathrm{d} x\right| \\
=\left|\frac{1}{|K|} \int_{K}\left[\frac{1}{k} \int_{t_{n-1}}^{t_{n}}\left[\partial_{t} \mathbf{U}(z)\right]_{h}(x) \mathrm{d} z\right] \mathrm{d} x\right| \leq\left\|\left[\partial_{t} \mathbf{U}\right]_{h}\right\|_{L^{\infty}\left(0, T ; L^{\infty}\left(\Omega ; \mathbb{R}^{3}\right)\right)} \leq\left\|\partial_{t} \mathbf{U}\right\|_{L^{\infty}\left(0, T ; L^{\infty}\left(\Omega ; \mathbb{R}^{3}\right)\right)},
\end{gathered}
$$

where we have used the property (9.20) of the projection onto the space $V_{h}(\Omega)$. Therefore, thanks to the mass conservation (3.15), we finally get

$$
\left|R_{2,1}^{n, K}\right| \leq \frac{M_{0}}{2}|K| k \| \partial_{t} \boldsymbol{U}_{L^{\infty}\left(0, T ; L^{\infty}\left(\Omega ; \mathbb{R}^{3}\right)\right)}^{2} .
$$

Let us now decompose the term $T_{2,1}$ as

$$
T_{2,1}=T_{2,2}+R_{2,2}, \text { with } T_{2,2}=k \sum_{n=1}^{m} \sum_{K \in \mathcal{T}}|K| \varrho_{K}^{n-1} \frac{\boldsymbol{U}_{h, K}^{n}-\boldsymbol{U}_{h, K}^{n-1}}{k} \cdot\left(\boldsymbol{U}_{h, K}^{n}-\boldsymbol{u}_{K}^{n}\right), \text { and } R_{2,2}=k \sum_{n=1}^{m} R_{2,2}^{n},
$$

where $R_{2,2}^{n}=\sum_{K \in \mathcal{T}}|K| \varrho_{K}^{n-1} \frac{\boldsymbol{U}_{h, K}^{n}-\boldsymbol{U}_{h, K}^{n-1}}{k} \cdot\left(\boldsymbol{U}_{h, K}^{n-1}-\boldsymbol{U}_{h, K}^{n}\right)-\sum_{K \in \mathcal{T}}|K| \varrho_{K}^{n-1} \frac{\boldsymbol{U}_{h, K}^{n}-\boldsymbol{U}_{h, K}^{n-1}}{k} \cdot\left(\boldsymbol{u}_{K}^{n-1}-\boldsymbol{u}_{K}^{n}\right)$.

By the same token as above, we may estimate the residual term as follows

$$
\left|R_{2,2}^{n}\right| \leq k c M_{0}\left\|\partial_{t} \boldsymbol{U}\right\|_{L^{\infty}\left(0, T ; W^{1, \infty}\left(\Omega ; \mathbb{R}^{3}\right)\right.}^{2}+c M_{0}^{1 / 2}\left(\sum_{K \in \mathcal{T}}|K| \varrho_{K}^{n-1}\left|\boldsymbol{u}_{K}^{n-1}-\boldsymbol{u}_{K}^{n}\right|^{2}\right)^{1 / 2} \| \partial_{t} \boldsymbol{U}_{L^{\infty}\left(0, T ; L^{\infty}\left(\Omega ; \mathbb{R}^{3}\right)\right)},
$$

where we have used the Hölder inequality to treat the second term; whence, by virtue of estimate (4.2a),

$$
\left|R_{2,2}\right| \leq \sqrt{k} c\left(M_{0}, E_{0},\left\|\left(\partial_{t} \boldsymbol{U}, \partial_{t} \nabla \boldsymbol{U}\right)\right\|_{L^{\infty}\left(Q_{T} ; \mathbb{R}^{12}\right)}\right)
$$

Step 2: Term $T_{3}$. Employing the definition (3.13) of upwind quantities, we easily establish that

$$
\begin{aligned}
& T_{3}=T_{3,1}+R_{3,1}, \\
& \text { with } T_{3,1}=k \sum_{n=1}^{m} \sum_{K \in \mathcal{T}} \sum_{\sigma \in \mathcal{E}(\mathcal{K})}|\sigma| \varrho_{\sigma}^{n, \text { up }}\left(\hat{\boldsymbol{u}}_{\sigma}^{n, \text { up }}-\hat{\boldsymbol{U}}_{h, \sigma}^{n, \mathrm{up}}\right) \cdot \boldsymbol{U}_{h, K}^{n} \boldsymbol{u}_{\sigma}^{n} \cdot \boldsymbol{n}_{\sigma, K}, \quad R_{3,1}=k \sum_{n=1}^{m} \sum_{\sigma \in \mathcal{E}_{\text {int }}} R_{3,1}^{n, \sigma}, \\
& \text { and } R_{3,1}^{n, \sigma}=|\sigma| \varrho_{K}^{n} \frac{\left|\boldsymbol{U}_{h, K}^{n}-\boldsymbol{U}_{h, L}^{n}\right|^{2}}{2}\left[\boldsymbol{u}_{\sigma}^{n} \cdot \boldsymbol{n}_{\sigma, K}\right]^{+}+|\sigma| \varrho_{L}^{n} \frac{\left|\boldsymbol{U}_{h, L}^{n}-\boldsymbol{U}_{h, K}^{n}\right|^{2}}{2}\left[\boldsymbol{u}_{\sigma}^{n} \cdot \boldsymbol{n}_{\sigma, L}\right]^{+}, \forall \sigma=K \mid L \in \mathcal{E}_{\text {int }} .
\end{aligned}
$$

Writing

$$
\boldsymbol{U}_{h, K}^{n}-\boldsymbol{U}_{h, L}^{n}=\boldsymbol{U}_{h, K}^{n}-\boldsymbol{U}_{h, \sigma}^{n}+\boldsymbol{U}_{h, \sigma}^{n}-\boldsymbol{U}_{h, L}^{n}, \sigma=K \mid L \in \mathcal{E}_{\text {int }},
$$

employing estimates (9.1) and (9.22) $s_{s=1}$ and the continuity of the mean value $\boldsymbol{U}_{\sigma}^{n}=\boldsymbol{U}_{h, \sigma}^{n}$ of $\boldsymbol{U}_{h}^{n}$ over faces $\sigma$, we infer by using the Taylor formula applied to function $x \mapsto U^{n}(x)$,

$$
\left|R_{3,1}^{n, \sigma}\right| \leq h^{2} c\|\nabla \boldsymbol{U}\|_{L^{\infty}\left(Q_{T} ; \mathbb{R}^{9}\right)}^{2}|\sigma|\left(\varrho_{K}^{n}+\varrho_{L}^{n}\right)\left|\boldsymbol{u}_{\sigma}^{n}\right|, \forall \sigma=K \mid L \in \mathcal{E}_{\text {int }}
$$


whence

$$
\begin{aligned}
\left|R_{3,1}\right| & \leq h c\|\nabla \boldsymbol{U}\|_{L^{\infty}\left(Q_{T} ; \mathbb{R}^{9}\right)}^{2}\left(\sum_{K \in \mathcal{T}} \sum_{\sigma=K \mid L \in \mathcal{E}(K)} h|\sigma|\left(\varrho_{K}^{n}+\varrho_{L}^{n}\right)^{6 / 5}\right)^{5 / 6}\left[k \sum_{n=1}^{m}\left(\sum_{K \in \mathcal{T}} \sum_{\sigma \in \mathcal{E}(K)} h\left|\sigma \| \boldsymbol{u}_{\sigma}^{n}\right|^{6}\right)^{1 / 3}\right]^{1 / 2} \\
& \leq h c\left(M_{0}, E_{0},\|\nabla \boldsymbol{U}\|_{L^{\infty}\left(Q_{T} ; \mathbb{R}^{9}\right)}\right)
\end{aligned}
$$

provided $\gamma \geq 6 / 5$, thanks to the discrete Hölder inequality, the equivalence relation (3.21), the equivalence of norms (9.35) and energy bounds listed in Corollary 4.1.

Evidently, for each face $\sigma=K \mid L \in \mathcal{E}_{\text {int }}, \boldsymbol{u}_{\sigma}^{n} \cdot \boldsymbol{n}_{\sigma, K}+\boldsymbol{u}_{\sigma}^{n} \cdot \mathbf{n}_{\sigma, L}=0$; whence, finally

$$
T_{3,1}=k \sum_{n=1}^{m} \sum_{K \in \mathcal{T}} \sum_{\sigma \in \mathcal{E}(K)}|\sigma| \varrho_{\sigma}^{n, \text { up }}\left(\hat{\boldsymbol{u}}_{\sigma}^{n, \text { up }}-\hat{\boldsymbol{U}}_{h, \sigma}^{n, \mathrm{up}}\right) \cdot\left(\boldsymbol{U}_{h, K}^{n}-\boldsymbol{U}_{\sigma}^{n}\right) \boldsymbol{u}_{\sigma}^{n} \cdot \boldsymbol{n}_{\sigma, K}
$$

Let us now decompose the term $T_{3,1}$ as

$$
\begin{aligned}
& T_{3,1}=T_{3,2}+R_{3,2}, \text { with } R_{3,2}=k \sum_{n=1}^{m} R_{3,2}^{n}, \\
& T_{3,2}=k \sum_{n=1}^{m} \sum_{K \in \mathcal{T}} \sum_{\sigma \in \mathcal{E}(K)}|\sigma| \varrho_{\sigma}^{n, \text { up }}\left(\hat{\boldsymbol{U}}_{h, \sigma}^{n, \text { up }}-\hat{\boldsymbol{u}}_{\sigma}^{n, \text { up }}\right) \cdot\left(\boldsymbol{U}_{\sigma}^{n}-\boldsymbol{U}_{h, K}^{n}\right) \hat{\boldsymbol{u}}_{\sigma}^{n, \text { up }} \cdot \boldsymbol{n}_{\sigma, K}, \text { and } \\
& R_{3,2}^{n}=\sum_{K \in \mathcal{T}} \sum_{\sigma \in \mathcal{E}(K)}|\sigma| \varrho_{\sigma}^{n, \text { up }}\left(\hat{\boldsymbol{U}}_{h, \sigma}^{n, \text { up }}-\hat{\boldsymbol{u}}_{\sigma}^{n, \text { up }}\right) \cdot\left(\boldsymbol{U}_{\sigma}^{n}-\boldsymbol{U}_{h, K}^{n}\right)\left(\boldsymbol{u}_{\sigma}^{n}-\hat{\boldsymbol{u}}_{\sigma}^{n, \text { up }}\right) \cdot \boldsymbol{n}_{\sigma, K} .
\end{aligned}
$$

By virtue of discrete Hölder's inequality and the first order Taylor formula applied to function $x \mapsto$ $\mathbf{U}^{n}(x)$ in order to evaluate the difference $\boldsymbol{U}_{\sigma}^{n}-\boldsymbol{U}_{h, K}^{n}$, we get

$$
\begin{aligned}
\left|R_{3,2}^{n}\right| & \leq c\|\nabla \mathbf{U}\|_{L^{\infty}\left(Q_{T} ; \mathbb{R}^{9}\right)}\left(\sum_{K \in \mathcal{T}} \sum_{\sigma \in \mathcal{E}(K)} h|\sigma| \varrho_{\sigma}^{n, \text { up }}\left|\hat{\boldsymbol{u}}_{\sigma}^{n, \text { up }}-\hat{\boldsymbol{U}}_{h, \sigma}^{n, \mathrm{up}}\right|^{2}\right)^{1 / 2} \\
& \times\left(\sum_{K \in \mathcal{T}} \sum_{\sigma \in \mathcal{E}(K)} h|\sigma|\left|\varrho_{\sigma}^{n, \mathrm{up}}\right|^{\gamma_{0}}\right)^{1 /\left(2 \gamma_{0}\right)}\left(\sum_{K \in \mathcal{T}} \sum_{\sigma \in \mathcal{E}(K)} h|\sigma|\left|\boldsymbol{u}_{\sigma}^{n}-\hat{\boldsymbol{u}}_{\sigma}^{n, \mathrm{up}}\right|^{q}\right)^{1 / q}
\end{aligned}
$$

where $\frac{1}{2}+\frac{1}{2 \gamma_{0}}+\frac{1}{q}=1, \gamma_{0}=\min \{\gamma, 2\}$ and $\gamma \geq 3 / 2$. For the sum in the last term of the above product, we have

$$
\begin{gathered}
\sum_{K \in \mathcal{T}} \sum_{\sigma \in \mathcal{E}(K)} h|\sigma|\left|\boldsymbol{u}_{\sigma}^{n}-\hat{\boldsymbol{u}}_{\sigma}^{n, \mathrm{up}}\right|^{q} \leq c \sum_{K \in \mathcal{T}} \sum_{\sigma \in \mathcal{E}(K)} h|\sigma|\left|\boldsymbol{u}_{\sigma}^{n}-\boldsymbol{u}_{K}^{n}\right|^{q} \\
\leq c\left(\sum_{K \in \mathcal{T}} \sum_{\sigma \in \mathcal{E}(K)}\left(\left\|\boldsymbol{u}_{\sigma}^{n}-\boldsymbol{u}^{n}\right\|_{L^{q}\left(K ; \mathbb{R}^{3}\right)}^{q}+\sum_{K \in \mathcal{T}}\left\|\boldsymbol{u}^{n}-\boldsymbol{u}_{K}^{n}\right\|_{L^{q}\left(K ; \mathbb{R}^{3}\right)}^{q}\right) \leq c h^{\frac{2 \gamma_{0}-3}{2 \gamma_{0}} q}\left|\boldsymbol{u}^{n}\right|_{V_{h}^{2}\left(\Omega ; \mathbb{R}^{3}\right)}^{q},\right.
\end{gathered}
$$

where we have used the definition (3.13), the Minkowski inequality and the interpolation inequalities (9.18 9.19). Now we can go back to the estimate of $R_{3,2}^{n}$ taking into account the upper bounds (4.9), (4.12 4.13), in order to get

$$
\left|R_{3,2}\right| \leq h^{A} c\left(M_{0}, E_{0},\|\nabla \mathbf{U}\|_{L^{\infty}\left(Q_{T} ; \mathbb{R}^{9}\right)}\right)
$$

provided $\gamma \geq 3 / 2$, where $A$ is given in (6.2).

Finally, we rewrite term $T_{3,2}$ as

$$
\begin{aligned}
& T_{3,2}=T_{3,3}+R_{3,3}, \text { with } R_{3,3}=k \sum_{n=1}^{m} R_{3,3}^{n}, \\
& T_{3,3}=k \sum_{n=1}^{m} \sum_{K \in \mathcal{T}} \sum_{\sigma \in \mathcal{E}(K)}|\sigma| \varrho_{\sigma}^{n, \text { up }}\left(\hat{\boldsymbol{U}}_{h, \sigma}^{n, \text { up }}-\hat{\boldsymbol{u}}_{\sigma}^{n, \text { up }}\right) \cdot\left(\boldsymbol{U}_{\sigma}^{n}-\boldsymbol{U}_{h, K}^{n}\right) \hat{\boldsymbol{U}}_{h, \sigma}^{n, \text { up }} \cdot \boldsymbol{n}_{\sigma, K}, \text { and } \\
& R_{3,3}^{n}=\sum_{K \in \mathcal{T}} \sum_{\sigma \in \mathcal{E}(K)}|\sigma| \varrho_{\sigma}^{n, \text { up }}\left(\hat{\boldsymbol{U}}_{h, \sigma}^{n, \text { up }}-\hat{\boldsymbol{u}}_{\sigma}^{n, \text { up }}\right) \cdot\left(\boldsymbol{U}_{\sigma}^{n}-\boldsymbol{U}_{h, K}^{n}\right)\left(\hat{\boldsymbol{u}}_{\sigma}^{n, \text { up }}-\hat{\boldsymbol{U}}_{h, \sigma}^{n, \text { up }}\right) \cdot \boldsymbol{n}_{\sigma, K} ;
\end{aligned}
$$


whence

$$
\left|R_{3,3}\right| \leq c\left(\|\nabla \boldsymbol{U}\|_{L^{\infty}\left(Q_{T}, \mathbb{R}^{9}\right)}\right) k \sum_{n=1}^{m} \mathcal{E}\left(\varrho^{n}, \mathbf{u}^{n} \mid r^{n}, \boldsymbol{U}^{n}\right)
$$

Step 3: Term $T_{4}$. Using the Stokes formula and the property (9.23) in Lemma 9.2, we easily see that

$$
T_{4}=-k \sum_{n=1}^{m} \sum_{K \in \mathcal{T}} \int_{\mathrm{K}} p\left(\varrho_{K}^{n}\right) \operatorname{div} \boldsymbol{U}^{n} \mathrm{~d} x
$$

Step 4: Term $T_{5}$. Using the Taylor formula, we get

$$
H^{\prime}\left(r_{K}^{n}\right)-H^{\prime}\left(r_{K}^{n-1}\right)=H^{\prime \prime}\left(r_{K}^{n}\right)\left(r_{K}^{n}-r_{K}^{n-1}\right)-\frac{1}{2} H^{\prime \prime \prime}\left(\bar{r}_{K}^{n}\right)\left(r_{K}^{n}-r_{K}^{n-1}\right)^{2},
$$

where $\bar{r}_{K}^{n} \in\left[\min \left(r_{K}^{n-1}, r_{K}^{n}\right), \max \left(r_{K}^{n-1}, r_{K}^{n}\right)\right] ;$ we infer

$$
\begin{aligned}
& T_{5}=T_{5,1}+R_{5,1}, \text { with } T_{5,1}=k \sum_{n=1}^{m} \sum_{K \in \mathcal{T}}|K|\left(r_{K}^{n}-\varrho_{K}^{n}\right) \frac{p^{\prime}\left(r_{K}^{n}\right)}{r_{K}^{n}} \frac{r_{K}^{n}-r_{K}^{n-1}}{k}, R_{5,1}=k \sum_{n=1}^{m} \sum_{K \in \mathcal{T}} R_{5,1}^{n, K}, \text { and } \\
& R_{5,1}^{n, K}=\frac{1}{2}|K| H^{\prime \prime \prime}\left(\bar{r}_{K}^{n}\right) \frac{\left(r_{K}^{n}-r_{K}^{n-1}\right)^{2}}{k}\left(\varrho_{K}^{n}-r_{K}^{n}\right) .
\end{aligned}
$$

Consequently, by the first order Taylor formula applied to function $t \mapsto r(t, x)$ on the interval $\left(t_{n-1}, t_{n}\right)$ and thanks to the mass conservation (3.15)

$$
\left|R_{5,1}\right| \leq k c\left(M_{0}, \underline{r}, \bar{r},\left|p^{\prime}\right|_{C^{1}([\underline{r}, \bar{r}]},\left\|\partial_{t} r\right\|_{L^{\infty}\left(Q_{T}\right)}\right),
$$

where $\underline{r}, \bar{r}$ are defined in (3.18).

Let us now decompose $T_{5,1}$ as follows:

$$
\begin{aligned}
& T_{5,1}=T_{5,2}+R_{5,2}, \text { with } T_{5,2}=k \sum_{n=1}^{m} \sum_{K \in \mathcal{T}} \int_{K}\left(r_{K}^{n}-\varrho_{K}^{n}\right) \frac{p^{\prime}\left(r_{K}^{n}\right)}{r_{K}^{n}}\left[\partial_{t} r\right]^{n} \mathrm{~d} x, R_{5,2}=k \sum_{n=1}^{m} \sum_{K \in \mathcal{T}} R_{5,2}^{n, K}, \text { and } \\
& R_{5,2}^{n, K}=\int_{K}\left(r_{K}^{n}-\varrho_{K}^{n}\right) \frac{p^{\prime}\left(r_{K}^{n}\right)}{r_{K}^{n}}\left(\frac{r_{K}^{n}-r_{K}^{n-1}}{k}-\left[\partial_{t} r\right]^{n}\right) \mathrm{d} x .
\end{aligned}
$$

In accordance with (3.17), here and in the sequel, $\left[\partial_{t} r\right]^{n}(x)=\partial_{t} r\left(t_{n}, x\right)$. We write using twice the Taylor formula in the integral form and the Fubini theorem,

$$
\begin{aligned}
\left|R_{5,2}^{n, K}\right| & =\frac{1}{k}\left|p^{\prime}\left(r_{K}^{n}\right) r_{K}^{n}\left(\varrho_{K}^{n}-r_{K}^{n}\right) \int_{K} \int_{t_{n-1}}^{t_{n}} \int_{s}^{t_{n}} \partial_{t}^{2} r(z) \mathrm{d} z \mathrm{~d} s \mathrm{~d} x\right| \\
& \leq \frac{p^{\prime}\left(r_{K}^{n}\right)}{r_{K}^{n}} \int_{t_{n-1}}^{t_{n}} \int_{K}\left|\varrho_{K}^{n}-r_{K}^{n}\right|\left|\partial_{t}^{2} r(z)\right| \mathrm{d} x \mathrm{~d} z \mathrm{~d} s \\
\leq & \frac{p^{\prime}\left(r_{K}^{n}\right)}{r_{K}^{n}}\left\|\varrho^{n}-\hat{r}^{n}\right\|_{L^{\gamma}(K)} \int_{t_{n-1}}^{t_{n}}\left\|\partial_{t}^{2} r(z)\right\|_{L^{\gamma^{\prime}(K)}} \mathrm{dzds} .
\end{aligned}
$$

Therefore, by virtue of Corollary 4.1, we have estimate

$$
\left|R_{5,2}\right| \leq k c\left(M_{0}, E_{0}, \underline{r}, \bar{r},\left|p^{\prime}\right|_{C^{1}([\underline{r}, \bar{r}]},\left\|\partial_{t}^{2} r\right\|_{L^{1}\left(0, T ; L \gamma^{\prime}(\Omega)\right.}\right) .
$$


Step 5: Term $T_{6}$. Using the same argumentation as in formula (6.7), we may write

$$
\begin{aligned}
& T_{6}=T_{6,1}+R_{6,1}, \quad R_{6,1}=k \sum_{n=1}^{m} \sum_{K \in \mathcal{T}} \sum_{\sigma \in \mathcal{E}(K)} R_{6,1}^{n, \sigma, K}, \text { with } \\
& T_{6,1}=k \sum_{n=1}^{m} \sum_{K \in \mathcal{T}} \sum_{\sigma=K \mid L \in \mathcal{E}(K)}|\sigma| \varrho_{K}^{n}\left(H^{\prime}\left(r_{K}^{n-1}\right)-H^{\prime}\left(r_{\sigma}^{n-1}\right)\right) \boldsymbol{u}_{\sigma}^{n} \cdot \boldsymbol{n}_{\sigma, K} \text {, and } \\
& R_{6,1}^{n, \sigma, K}=|\sigma|\left(\varrho_{\sigma}^{n, \text { up }}-\varrho_{K}^{n}\right)\left(H^{\prime}\left(r_{K}^{n-1}\right)-H^{\prime}\left(r_{\sigma}^{n-1}\right)\right) \boldsymbol{u}_{\sigma}^{n} \cdot \boldsymbol{n}_{\sigma, K}, \text { for } \sigma=K \mid L .
\end{aligned}
$$

We estimate this term separately for $\gamma \leq 2$ and $\gamma>2$. If $\gamma \leq 2$, motivated by Lemma 4.2, we may write

$$
\begin{aligned}
\left|R_{6,1}^{n, \sigma, K}\right| \leq \sqrt{h} & \left\|\nabla H^{\prime}(r)\right\|_{L^{\infty}\left(Q_{T} ; \mathbb{R}^{3}\right)}|\sigma| \\
& \times\left(\frac{\left|\varrho_{\sigma}^{n, \text { up }}-\varrho_{K}^{n}\right|}{\max \left(\varrho_{K}, \varrho_{L}\right)^{(2-\gamma) / 2} \sqrt{\mid \boldsymbol{u}_{\sigma}^{n} \cdot \boldsymbol{n}_{\sigma, K}} \mid} 1_{\varrho_{\sigma}^{n} \geq 1} \sqrt{h}\left(\varrho_{K}^{n}+\varrho_{L}^{n}\right)^{(2-\gamma) / 2} \sqrt{\left|\boldsymbol{u}_{\sigma}^{n} \cdot \boldsymbol{n}_{\sigma, K}\right|}\right. \\
& \left.+\left|\varrho_{\sigma}^{n, \text { up }}-\varrho_{K}^{n}\right| \sqrt{\left|\boldsymbol{u}_{\sigma}^{n} \cdot \boldsymbol{n}_{\sigma, K}\right|} 1_{\varrho_{\sigma}^{n}<1} \sqrt{h} \sqrt{\left|\boldsymbol{u}_{\sigma}^{n} \cdot \boldsymbol{n}_{\sigma, K}\right|}\right),
\end{aligned}
$$

where we again use the first order Taylor formula applied to function $H^{\prime}$ between endpoints $r_{K}^{n-1}, r_{\sigma}^{n-1}$, and where the numbers $\varrho_{\sigma}^{n}$ are defined in Lemma 4.1. Consequently, an application of the Hölder and Young inequalities yields

$$
\begin{aligned}
& \left|R_{6,1}\right| \leq \sqrt{h} c\left\|\nabla H^{\prime}(r)\right\|_{L^{\infty}\left(Q_{T} ; \mathbb{R}^{3}\right)} k \sum_{n=1}^{m}\left[\left(\sum_{K \in \mathcal{T}} \sum_{\sigma=K \mid L \in \mathcal{E}(K)}|\sigma| \frac{\left(\varrho_{\sigma}^{n, \text { up }}-\varrho_{K}^{n}\right)^{2}}{\max \left(\varrho_{K}, \varrho_{L}\right)^{(2-\gamma)}}\left|\boldsymbol{u}_{\sigma}^{n} \cdot \boldsymbol{n}_{\sigma, K}\right| 1_{\varrho_{\sigma}^{n} \geq 1}\right)^{1 / 2}\right. \\
& \times\left(\sum_{K \in \mathcal{T}} \sum_{\sigma \in \mathcal{E}(K)}|\sigma| h \varrho_{K}^{2-\gamma}\left|\boldsymbol{u}_{\sigma}^{n} \cdot \boldsymbol{n}_{\sigma, K}\right|\right)^{1 / 2} \\
& \left.+\left(\sum_{K \in \mathcal{T}} \sum_{\sigma=K \mid L \in \mathcal{E}(K)}|\sigma| h\left(\varrho_{\sigma}^{n \text {,up }}-\varrho_{K}^{n}\right)^{2}\left|\boldsymbol{u}_{\sigma}^{n} \cdot \boldsymbol{n}_{\sigma, K}\right| 1_{\varrho_{\sigma}^{n}<1}\right)^{1 / 2}\left(\sum_{K \in \mathcal{T}} \sum_{\sigma \in \mathcal{E}(K)}|\sigma| h\left|\boldsymbol{u}_{\sigma}^{n} \cdot \boldsymbol{n}_{\sigma, K}\right|\right)^{1 / 2}\right] \\
& \leq \sqrt{h} c\left\|\nabla H^{\prime}(r)\right\|_{L^{\infty}\left(Q_{T} ; \mathbb{R}^{3}\right)} k \sum_{n=1}^{m}\left[\left(\sum_{K \in \mathcal{T}} \sum_{\sigma=K \mid L \in \mathcal{E}(K)}|\sigma| \frac{\left(\varrho_{\sigma}^{n, \text { up }}-\varrho_{K}^{n}\right)^{2}}{\max \left(\varrho_{K}, \varrho_{L}\right)^{(2-\gamma)}}\left|\boldsymbol{u}_{\sigma}^{n} \cdot \boldsymbol{n}_{\sigma, K}\right| 1_{\varrho_{\sigma}^{n} \geq 1}\right.\right. \\
& +\left(\sum_{K \in \mathcal{T}}|K| \varrho_{K}^{6(2-\gamma) / 5}\right)^{5 / 6}\left(\sum_{\sigma \in \mathcal{E}}|\sigma| h\left|\boldsymbol{u}_{\sigma}^{n}\right|^{6}\right)^{1 / 6} \\
& \left.+\sum_{K \in \mathcal{T}} \sum_{\sigma=K \mid L \in \mathcal{E}(K)}|\sigma| h\left(\varrho_{\sigma}^{n, \text { up }}-\varrho_{K}^{n}\right)^{2}\left|\boldsymbol{u}_{\sigma}^{n} \cdot \boldsymbol{n}_{\sigma, K}\right| 1_{\bar{\varrho}_{K}^{n}<1}+|\Omega|^{5 / 6}\left(\sum_{\sigma \in \mathcal{E}}|\sigma| h\left|\boldsymbol{u}_{\sigma}^{n}\right|^{6}\right)^{1 / 6}\right]
\end{aligned}
$$

We deduce employing the discrete Hölder inequality

$$
\begin{gathered}
k \sum_{n=1}^{m}\left(\sum_{K \in \mathcal{T}}|K| \varrho_{K}^{6(2-\gamma) / 5}\right)^{5 / 6}\left(\sum_{\sigma \in \mathcal{E}}|\sigma| h\left|\boldsymbol{u}_{\sigma}^{n}\right|^{6}\right)^{1 / 6} \\
\leq\left[k \sum_{n=1}^{m}\left(\sum_{K \in \mathcal{T}}|K| \varrho_{K}^{6(2-\gamma) / 5}\right)^{5 / 3}\right]^{1 / 2}\left[k \sum_{n=1}^{m}\left(\sum_{\sigma \in \mathcal{E}}|\sigma| h\left|\boldsymbol{u}_{\sigma}^{n}\right|^{6}\right)^{1 / 3}\right]^{1 / 2}
\end{gathered}
$$

and

$$
k \sum_{n=1}^{m}\left(\sum_{\sigma \in \mathcal{E}}|\sigma| h\left|\boldsymbol{u}_{\sigma}^{n}\right|^{6}\right)^{1 / 6} \leq \sqrt{T}\left[k \sum_{n=1}^{m}\left(\sum_{\sigma \in \mathcal{E}}|\sigma| h\left|\boldsymbol{u}_{\sigma}^{n}\right|^{6}\right)^{1 / 3}\right]^{1 / 2}
$$

Coming back to (6.17) we deduce that

$$
\left|R_{6,1}\right| \leq \sqrt{h} c\left(M_{0}, E_{0}, \underline{r}, \bar{r},\left|p^{\prime}\right|_{C([\underline{r}, \bar{r}])},\|\nabla r\|_{L^{\infty}\left(Q_{T} ; \mathbb{R}^{3}\right)}\right)
$$


provided $\gamma \geq 12 / 11$, where we use estimate (4.16), estimates (4.10), (4.12) of Corollary 4.1 and equivalence relation (9.35). In the case $\gamma>2$, the same final bound may be obtained by a similar argument, replacing the estimate (4.16) by (4.15).

Let us now decompose the term $T_{6,1}$ as

$$
\begin{aligned}
& T_{6,1}=T_{6,2}+R_{6,2}, \text { with } T_{6,2}=k \sum_{n=1}^{m} \sum_{K \in \mathcal{T}} \sum_{\sigma=K \mid L \in \mathcal{E}(K)}|\sigma| \varrho_{K}^{n} H^{\prime \prime}\left(r_{K}^{n-1}\right)\left(r_{K}^{n-1}-r_{\sigma}^{n-1}\right)\left[\boldsymbol{u}_{\sigma}^{n} \cdot \boldsymbol{n}_{\sigma, K}\right], \\
& R_{6,2}=k \sum_{n=1}^{m} \sum_{K \in \mathcal{K}} \sum_{\sigma \in \mathcal{E}(K)} R_{6,2}^{n, \sigma, K}, \text { and } \\
& R_{6,2}^{n, \sigma, K}=|\sigma| \varrho_{K}^{n}\left(H^{\prime}\left(r_{K}^{n-1}\right)-H^{\prime}\left(r_{\sigma}^{n-1}\right)-H^{\prime \prime}\left(r_{K}^{n-1}\right)\left(r_{K}^{n-1}-r_{\sigma}^{n-1}\right)\right)\left[\boldsymbol{u}_{\sigma}^{n} \cdot \boldsymbol{n}_{\sigma, K}\right]
\end{aligned}
$$

Therefore, by virtue of the second order Taylor formula applied to function H', Hölder's inequality, (9.30), (9.35), and (4.9), (4.12) in Corollary 4.1, we have, provided $\gamma \geq 6 / 5$,

$$
\begin{aligned}
\left|R_{6,2}\right| & \leq h c\left(\left|H^{\prime \prime}\right|_{C([\underline{r}, \bar{r}])}+\left|H^{\prime \prime \prime}\right|_{C([\underline{r}, \bar{r}])}\right)\|\nabla r\|_{L^{\infty}\left(Q_{T} ; \mathbb{R}^{3}\right)}\|\varrho\|_{L^{\infty}\left(0, T ; L^{\gamma}(\Omega)\right)}\|\boldsymbol{u}\|_{L^{2}\left(0, T ; V_{h}^{2}\left(\Omega ; \mathbb{R}^{3}\right)\right)} \\
& \leq h c\left(M_{0}, E_{0}, \underline{r}, \bar{r},\left|p^{\prime}\right|_{C^{1}([\underline{r}, \bar{r}])},\|\nabla r\|_{L^{\infty}\left(Q_{T} ; \mathbb{R}^{3}\right)}\right),
\end{aligned}
$$

where in the first line we have used notation (3.11).

Let us now deal with the term $T_{6,2}$. Noting that $\int_{K} \nabla r^{n-1} \mathrm{~d} x=\sum_{\sigma \in \mathcal{E}(K)}|\sigma|\left(r_{\sigma}^{n-1}-r_{K}^{n-1}\right) \boldsymbol{n}_{\sigma, K}$, we may write

$$
\begin{aligned}
\sum_{\sigma \in \mathcal{E}(K)} \mid & \sigma \mid \varrho_{K}^{n} H^{\prime \prime}\left(r_{K}^{n-1}\right)\left(r_{K}^{n-1}-r_{\sigma}^{n-1}\right)\left[\boldsymbol{u}_{\sigma}^{n} \cdot \boldsymbol{n}_{\sigma, K}\right] \\
& =-\int_{K} \varrho_{K}^{n} H^{\prime \prime}\left(r_{K}^{n-1}\right) \boldsymbol{u}_{K}^{n} \cdot \nabla r^{n-1} \mathrm{~d} x+\sum_{\sigma \in \mathcal{E}(K)}|\sigma| \varrho_{K}^{n} H^{\prime \prime}\left(r_{K}^{n-1}\right)\left(r_{K}^{n-1}-r_{\sigma}^{n-1}\right)\left(\boldsymbol{u}_{\sigma}^{n}-\boldsymbol{u}_{K}^{n}\right) \cdot \boldsymbol{n}_{\sigma, K} .
\end{aligned}
$$

Consequently, $T_{6,2}=T_{6,3}+R_{6,3}$, with

$$
\begin{aligned}
& T_{6,3}=-k \sum_{n=1}^{m} \sum_{K \in \mathcal{T}} \int_{K} \varrho_{K}^{n} H^{\prime \prime}\left(r_{K}^{n-1}\right) \boldsymbol{u}^{n} \cdot \nabla r^{n-1} \mathrm{~d} x, \\
& R_{6,3}=k \sum_{n=1}^{m} \sum_{K \in \mathcal{T}} \int_{K} \varrho_{K}^{n} H^{\prime \prime}\left(r_{K}^{n-1}\right)\left(\boldsymbol{u}^{n}-\boldsymbol{u}_{K}^{n}\right) \cdot \nabla r^{n-1} \mathrm{~d} x \\
& \quad+k \sum_{n=1}^{m} \sum_{K \in \mathcal{T}} \sum_{\sigma \in \mathcal{E}(K)}|\sigma| \varrho_{K}^{n} H^{\prime \prime}\left(r_{K}^{n-1}\right)\left(r_{K}^{n-1}-r_{\sigma}^{n-1}\right)\left(\boldsymbol{u}_{\sigma}^{n}-\boldsymbol{u}_{K}^{n}\right) \cdot \boldsymbol{n}_{\sigma, K}, \\
& \left|R_{6,3}\right| \leq c\left\|H^{\prime \prime}(r) \nabla r\right\|_{L^{\infty}\left(Q_{T} ; \mathbb{R}^{3}\right)}\left[k \sum_{n=1}^{m} \sum_{K \in \mathcal{T}}\left\|\varrho_{K}^{n}\right\|_{L^{\gamma_{0}(K)}}\left\|\boldsymbol{u}^{n}-\boldsymbol{u}_{K}^{n}\right\|_{L^{\gamma_{0}^{\prime}}\left(K ; \mathbb{R}^{3}\right)}+\right. \\
& \left.\quad k \sum_{n=1}^{m} \sum_{K \in \mathcal{T}} \sum_{\sigma \in \mathcal{E}(K)}\left\|\varrho_{K}^{n}\right\|_{L^{\gamma_{0}(K)}}\left\|\boldsymbol{u}_{\sigma}^{n}-\boldsymbol{u}_{K}^{n}\right\|_{L^{\gamma_{0}^{\prime}\left(K ; \mathbb{R}^{3}\right)}}\right], \quad \gamma_{0}=\min \{\gamma, 2\},
\end{aligned}
$$

where we have used the Hölder inequality, and also the Taylor formula applied to function $x \mapsto r\left(t_{n-1}, x\right)$ together with equivalence relation (3.21) yielding $|\sigma| h \leq|K|$, to treat the second term. Consequently, by virtue of Hölder's inequality, interpolation inequality (9.5) (to estimate $\left\|\mathbf{u}^{n}-\mathbf{u}_{K}^{n}\right\|_{L^{\gamma_{0}^{\prime}\left(K ; \mathbb{R}^{3}\right)}}$ by 
$\left.h^{\left(5 \gamma_{0}-6\right) /\left(2 \gamma_{0}\right)}\left\|\nabla_{x} \mathbf{u}^{n}\right\|_{L^{2}\left(K ; \mathbb{R}^{9}\right)}, \gamma_{0}=\min \{\gamma, 2\}\right)$ in the first term, and by the the Hölder inequality and (9.5 9.6) (to estimate $\left\|\mathbf{u}_{\sigma}^{n}-\mathbf{u}_{K}^{n}\right\|_{L^{\gamma^{\prime}\left(K ; \mathbb{R}^{3}\right)}}$ by $h^{\left(5 \gamma_{0}-6\right) /\left(2 \gamma_{0}\right)}\left\|\nabla_{x} \mathbf{u}^{n}\right\|_{L^{2}\left(K ; \mathbb{R}^{9}\right)}$ ) in the second term, we get

$$
\begin{gathered}
\left|R_{6,3}\right| \leq c h^{\left(5 \gamma_{0}-6\right) /\left(2 \gamma_{0}\right)}\left\|H^{\prime \prime}(r) \nabla r\right\|_{L^{\infty}\left(Q_{T} ; \mathbb{R}^{3}\right)} k \sum_{n=1}^{m}\left(\sum_{K \in \mathcal{T}}\left\|\varrho_{K}^{n}\right\|_{L^{\gamma_{0}(K)}}^{2}\right)^{1 / 2}\left(\sum_{K \in \mathcal{T}}\left\|\nabla_{x} \boldsymbol{u}^{n}\right\|_{L^{2}\left(K ; \mathbb{R}^{9}\right)}^{2}\right)^{1 / 2} \\
\leq c h^{\left(5 \gamma_{0}-6\right) /\left(2 \gamma_{0}\right)}\left\|H^{\prime \prime}(r) \nabla r\right\|_{L^{\infty}\left(Q_{T} ; \mathbb{R}^{3}\right)} k \sum_{n=1}^{m}\left(\sum_{K \in \mathcal{T}}\left\|\varrho_{K}^{n}\right\|_{L^{\gamma_{0}(K)}}^{\gamma_{0}}\right)^{1 / \gamma_{0}}\left(\sum_{K \in \mathcal{T}}\left\|\nabla_{x} \boldsymbol{u}^{n}\right\|_{L^{2}\left(K ; \mathbb{R}^{9}\right)}^{2}\right)^{1 / 2}
\end{gathered}
$$

provided $\gamma \geq 6 / 5$, where we have used the discrete Hölder inequality and the algebraic inequality (9.7). Now it remains to use (4.9), (4.12) in Corollary 4.1 in order to get

$$
\left|R_{6,3}\right| \leq h^{A} c\left(M_{0}, E_{0}, \underline{r}, \bar{r},\left|p^{\prime}\right|_{C^{1}([\underline{r}, \bar{r}])}\|\nabla r\|_{L^{\infty}\left(Q_{T} ; \mathbb{R}^{3}\right)}\right),
$$

where $A$ is defined in (6.2).

Finally we write $T_{6,3}=T_{6,4}+R_{6,4}$, with

$$
\begin{aligned}
& T_{6,4}=-k \sum_{n=1}^{m} \sum_{K \in \mathcal{T}} \int_{K} \varrho_{K}^{n} \frac{p^{\prime}\left(r_{K}^{n}\right)}{r_{K}^{n}} \boldsymbol{u}^{n} \cdot \nabla r^{n} \mathrm{~d} x, \\
& R_{6,4}=k \sum_{n=1}^{m} \sum_{K \in \mathcal{T}} \int_{K} \varrho_{K}^{n}\left(H^{\prime \prime}\left(r_{K}^{n}\right) \nabla r^{n}-H^{\prime \prime}\left(r_{K}^{n-1}\right) \nabla r^{n-1}\right) \cdot \boldsymbol{u}^{n} \mathrm{~d} x,
\end{aligned}
$$

where by the same token as in (6.14),

$$
\left|R_{6,4}\right| \leq k c\left(M_{0}, E_{0}, \underline{r}, \bar{r},\left|p^{\prime}\right|_{C^{1}([\underline{r}, \bar{r}])},\left\|\nabla r, \partial_{t} r\right\|_{L^{\infty}\left(Q_{T} ; \mathbb{R}^{4}\right)},\left\|\partial_{t} \nabla r\right\|_{L^{2}\left(0, T ; L^{\left.6 \gamma /(5 \gamma-6)\left(\Omega ; \mathbb{R}^{3}\right)\right)}\right)} .\right.
$$

We are now in position to conclude the proof of Lemma 6.1; we obtain the inequality (6.1) by gathering the principal terms (6.4), (6.9), (6.11), (6.13), (6.21) and the residual terms estimated in (6.3), (6.5), (6.6), (6.8), (6.10), (6.12), (6.14), (6.16), (6.17), (6.19), (6.20), (6.22) at the right hand side $\sum_{i=1}^{6} T_{i}$ of the discrete relative energy inequality (5.1).

\section{7 - A discrete identity satisfied by the strong solution}

This section is devoted to the proof of a discrete identity satisfied by any strong solution. This identity is stated in Lemma 7.1 below. It will be used in combination with the approximate relative energy inequality stated in Lemma 6.1 to deduce the convenient form of the relative energy inequality verified by any function being a strong solution to the compressible Navier-Stokes system. This last step is performed in the next section.

Lemma 7.1 (A discrete identity for strong solutions). Suppose that $\Omega \subset \mathbb{R}^{3}$ is a bounded polyhedral domain and $\mathcal{T}$ a regular triangulation introduced in Section [3.1. Let the pressure $p$ be a $C^{2}(0, \infty)$ function satisfying hypotheses (1.5) and (1.6) with $\gamma \geq 3 / 2$. Let $(r, \boldsymbol{U})$ belong to the class (3.18) satisfy equation (1.1) with the viscosity coefficients $\mu, \lambda$ obeying (1.4).

Let $\left(\varrho^{0}, \boldsymbol{u}^{0}\right) \in L_{h}^{+}(\Omega) \times \boldsymbol{W}_{h}(\Omega)$ and suppose that $\left(\varrho^{n}\right)_{1 \leq n \leq N} \in\left[L_{h}^{+}(\Omega)\right]^{N},\left(\boldsymbol{u}^{n}\right)_{1 \leq n \leq N} \in\left[\boldsymbol{W}_{h}(\Omega)\right]^{N}$ is a solution of the discrete problem (3.14). Then there exists

$$
\left.c=c\left(M_{0}, E_{0}, \underline{r}, \bar{r},\left|p^{\prime}\right|_{C^{1}([\underline{r}, \bar{r}])},\left\|\left(\nabla r, \partial_{t} r, \boldsymbol{U}, \nabla \boldsymbol{U}, \nabla^{2} \boldsymbol{U}, \partial_{t} \boldsymbol{U}, \partial_{t}^{2} \boldsymbol{U}, \partial_{t} \nabla \boldsymbol{U},\right)\right\|_{L^{\infty}\left(Q_{T} ; \mathbb{R}^{58}\right)}\right)\right)>0,
$$


such that for any $m=1, \ldots, N$, the following identity holds:

$$
\begin{aligned}
& k \sum_{n=1}^{m} \sum_{K \in \mathcal{T}} \int_{K}\left(\mu \nabla \boldsymbol{U}_{h}^{n} \cdot \nabla\left(\boldsymbol{u}^{n}-\boldsymbol{U}_{h}^{n}\right)+(\mu+\lambda) \operatorname{div} \boldsymbol{U}_{h}^{n} \operatorname{div}\left(\boldsymbol{u}^{n}-\boldsymbol{U}_{h}^{n}\right)\right) \mathrm{d} x \\
& \quad+k \sum_{n=1}^{m} \sum_{K \in \mathcal{T}} \int_{K} r_{K}^{n-1} \frac{\boldsymbol{U}_{h, K}^{n}-\boldsymbol{U}_{h, K}^{n-1}}{k} \cdot\left(\boldsymbol{u}_{K}^{n}-\boldsymbol{U}_{h, K}^{n}\right) \mathrm{d} x \\
& \quad+k \sum_{n=1}^{m} \sum_{K \in \mathcal{T}} \sum_{\sigma \in \mathcal{E}(K)}|\sigma| \hat{r}_{\sigma}^{n, \text { up }}\left[\hat{\boldsymbol{U}}_{h, \sigma}^{n, \text { up }} \cdot \mathbf{n}_{\sigma, K}\right]\left(\boldsymbol{U}_{\sigma}^{n}-\boldsymbol{U}_{h, K}^{n}\right) \cdot\left(\hat{\boldsymbol{u}}_{\sigma}^{n, \text { up }}-\hat{\boldsymbol{U}}_{h, \sigma}^{n, \text { up }}\right) \\
& \quad+k \sum_{n=1}^{m} \sum_{K \in \mathcal{T}} \int_{K} p\left(r_{K}^{n}\right) \operatorname{div} \boldsymbol{U}^{n} \mathrm{~d} x+k \sum_{n=1}^{m} \sum_{K \in \mathcal{T}} \int_{K} p^{\prime}\left(r_{K}^{n}\right) \boldsymbol{u}^{n} \cdot \nabla r^{n} \mathrm{~d} x+\mathcal{R}_{h, k}^{m}=0,
\end{aligned}
$$

where

$$
\left|\mathcal{R}_{h, k}^{m}\right| \leq c(h+k)
$$

and where we have used notation (3.17) for $r^{n}, \boldsymbol{U}^{n}$ and (3.7 3.8) for $\mathbf{U}_{h}^{n}, \mathbf{U}_{h, K}^{n}, r_{K}^{n}, \mathbf{u}_{\sigma}^{n}$.

Before starting the proof we recall an auxiliary algebraic inequality whose straightforward proof is left to the reader, and introduce some notations.

Lemma 7.2. Let $p$ satisfies assumptions (1.5) and (1.6). Let $0<a<b<\infty$. Then there exists $c=c(a, b)>0$ such that for all $\varrho \in[0, \infty)$ and $r \in[a, b]$ there holds

$$
E(\varrho \mid r) \geq c(a, b)\left(1_{R_{+} \backslash[a / 2,2 b]}(\varrho)+\varrho^{\gamma} 1_{R_{+} \backslash[a / 2,2 b]}(\varrho)+(\varrho-r)^{2} 1_{[a / 2,2 b]}(\varrho)\right),
$$

where $E(\varrho \mid r)$ is defined in (2.5).

If we take in Lemma $7.2 \varrho=\varrho^{n}(x), \varrho^{n} \in L_{h}^{+}(\Omega), r=\hat{r}^{n}(x), a=\underline{r}, b=\bar{r}$ (where $\mathrm{r}$ is a function belonging to class (3.18) and $\underline{r}, \bar{r}$ are its lower and upper bounds, respectively), we obtain

$$
E\left(\varrho^{n}(x) \mid \hat{r}^{n}(x)\right) \geq c(\underline{r}, \bar{r})\left(1_{\left.R_{+} \backslash \underline{r} / 2,2 \bar{r}\right]}\left(\varrho^{n}(x)\right)+\left(\varrho^{n}\right)^{\gamma}(x) 1_{\left.R_{+} \backslash \underline{r} / 2,2 \bar{r}\right]}\left(\varrho^{n}(x)\right)+\left(\varrho^{n}(x)-\hat{r}^{n}(x)\right)^{2} 1_{[\underline{r} / 2,2 \bar{r}]}\left(\varrho^{n}(x)\right)\right)
$$

Now, for fixed numbers $\underline{r}$ and $\bar{r}$ and fixed functions $\varrho^{n} \in L_{h}^{+}(\Omega), n=0, \ldots, N$, we introduce the residual and essential subsets of $\Omega$ (relative to $\varrho^{n}$ ) as follows:

$$
N_{\mathrm{ess}}^{n}=\left\{x \in \Omega \mid \frac{1}{2} \underline{\underline{r}} \leq \varrho^{n}(x) \leq 2 \bar{r}\right\}, N_{\mathrm{res}}^{n}=\Omega \backslash N_{\mathrm{ess}}^{n} .
$$

and we set

$$
[g]_{\mathrm{ess}}(x)=g(x) 1_{N_{\mathrm{ess}}^{n}}(x),[g]_{\mathrm{res}}(x)=g(x) 1_{N_{\mathrm{res}}^{n}}(x), \quad x \in \Omega, g \in L^{1}(\Omega) .
$$

Integrating inequality (7.2) we deduce

$$
c(\underline{r}, \bar{r}) \sum_{K \in \mathcal{T}} \int_{K}\left([1]_{\mathrm{res}}+\left[\left(\varrho^{n}\right)^{\gamma}\right]_{\mathrm{res}}+\left[\varrho^{n}-\hat{r}^{n}\right]_{\mathrm{ess}}^{2}\right) \mathrm{d} x \leq \mathcal{E}\left(\varrho^{n}, \boldsymbol{u}^{n} \mid r^{n}, \boldsymbol{U}^{n}\right) .
$$

for any pair $(r, \boldsymbol{U})$ belonging to the class (3.18) and any $\varrho^{n} \in L_{h}(\Omega)$.

We are now ready to proceed to the proof of Lemma 7.1 .

Proof. We start by projecting the momentum equation to the discrete spaces. Since $(r, \boldsymbol{U})$ satisfies (1.1) and belongs to the class (3.18), Equation (1.1b) can be rewritten in the form

$$
r \partial_{t} \boldsymbol{U}+r \boldsymbol{U} \cdot \nabla \boldsymbol{U}+\nabla p(r)=\mu \Delta \boldsymbol{U}+(\mu+\lambda) \nabla \operatorname{div} \boldsymbol{U} .
$$


We write equation (7.5) at $t=t_{n}$, multiply scalarly by $\boldsymbol{u}^{n}-\boldsymbol{U}_{h}^{n}$, and integrate over $\Omega$. We get, after summation from $n=1$ to $m$,

$$
\begin{array}{lll}
\sum_{i=1}^{5} \mathcal{T}_{i}=0, & \text { with } & \mathcal{T}_{1}=-k \sum_{n=1}^{m} \int_{\Omega}\left(\mu \Delta \boldsymbol{U}^{n}+(\mu+\lambda) \nabla \operatorname{div} \boldsymbol{U}^{n}\right) \cdot\left(\boldsymbol{u}^{n}-\boldsymbol{U}_{h}^{n}\right) \mathrm{d} x, \\
\mathcal{T}_{2}=k \sum_{n=1}^{m} \int_{\Omega} r^{n}\left[\partial_{t} \boldsymbol{U}\right]^{n} \cdot\left(\boldsymbol{u}^{n}-\boldsymbol{U}_{h}^{n}\right) \mathrm{d} x, & \mathcal{T}_{3}=k \sum_{n=1}^{m} \int_{\Omega} r^{n} \boldsymbol{U}^{n} \cdot \nabla \boldsymbol{U}^{n} \cdot\left(\boldsymbol{u}^{n}-\boldsymbol{U}_{h}^{n}\right) \mathrm{d} x \\
\mathcal{T}_{4}=k \sum_{n=1}^{m} \int_{\Omega} \nabla p\left(r^{n}\right) \cdot \boldsymbol{u}^{n} \mathrm{~d} x, & \mathcal{T}_{5}=-k \sum_{n=1}^{m} \int_{\Omega} \nabla p\left(r^{n}\right) \cdot \boldsymbol{U}_{h}^{n} \mathrm{~d} x .
\end{array}
$$

In the steps below, we deal with each of the terms $\mathcal{T}_{i}$.

Step 1: Term $\mathcal{T}_{1}$. Integrating by parts, we get:

$$
\begin{aligned}
& \mathcal{T}_{1}=\mathcal{T}_{1,1}+\mathcal{R}_{1,1}, \\
& \text { with } \mathcal{T}_{1,1}=k \sum_{n=1}^{m} \sum_{K \in \mathcal{T}} \int_{K}\left(\mu \nabla \boldsymbol{U}_{h}^{n}: \nabla\left(\boldsymbol{u}^{n}-\boldsymbol{U}_{h}^{n}\right)+(\mu+\lambda) \operatorname{div} \boldsymbol{U}_{h}^{n} \operatorname{div}\left(\boldsymbol{u}^{n}-\boldsymbol{U}_{h}^{n}\right)\right) \mathrm{d} x \\
& \text { and } \mathcal{R}_{1,1}=I_{1}+I_{2}, \text { with } \\
& I_{1}=k \sum_{n=1}^{m} \sum_{K \in \mathcal{T}} \int_{K}\left(\mu \nabla\left(\boldsymbol{U}^{n}-\boldsymbol{U}_{h}^{n}\right): \nabla\left(\boldsymbol{u}^{n}-\boldsymbol{U}_{h}^{n}\right)+(\mu+\lambda) \operatorname{div}\left(\left(\boldsymbol{U}^{n}-\boldsymbol{U}_{h}^{n}\right) \operatorname{div}\left(\boldsymbol{u}^{n}-\boldsymbol{U}_{h}^{n}\right)\right) \mathrm{d} x\right. \\
& I_{2}=-k \sum_{n=1}^{m} \sum_{K \in \mathcal{T}} \sum_{\sigma \in \mathcal{E}(K)} \int_{\sigma}\left(\mu \boldsymbol{n}_{\sigma, K} \cdot \nabla \boldsymbol{U}^{n} \cdot\left(\boldsymbol{u}^{n}-\boldsymbol{U}_{h}^{n}\right)+(\lambda+\mu) \operatorname{div} \boldsymbol{U}^{n}\left(\boldsymbol{u}^{n}-\boldsymbol{U}_{h}^{n}\right) \cdot \boldsymbol{n}_{\sigma, K}\right) \mathrm{d} S \\
& =-k \sum_{n=1}^{m} \sum_{\sigma \in \mathcal{E}} \int_{\sigma}\left(\mu \boldsymbol{n}_{\sigma} \cdot \nabla \boldsymbol{U}^{n} \cdot\left[\boldsymbol{u}^{n}-\boldsymbol{U}_{h}^{n}\right]_{\sigma, \mathbf{n}_{\sigma}}+(\lambda+\mu) \operatorname{div} \boldsymbol{U}^{n}\left[\boldsymbol{u}^{n}-\boldsymbol{U}_{h}^{n}\right]_{\sigma, \mathbf{n}_{\sigma}} \cdot \boldsymbol{n}_{\sigma}\right) \mathrm{d} S,
\end{aligned}
$$

where in the last line $\mathbf{n}_{\sigma}$ is a unit normal to $\sigma$ and $[\cdot]_{\sigma, \mathbf{n}_{\sigma}}$ is the jump over sigma (with respect to $\mathbf{n}_{\sigma}$ ) defined in Lemma 9.5. Employing estimate (9.22) we easily verify that

$$
\left|I_{1}\right| \leq h c\left(M_{0}, E_{0},\|\nabla \mathbf{U}\|_{L^{\infty}\left(0, T ; W^{1, \infty}(\Omega)\right)}\right) .
$$

Since the integral over any face $\sigma$ of the jump of a function from $V_{h}(\Omega)$ is zero, we may write

$$
\begin{aligned}
I_{2} & =-k \sum_{n=1}^{m} \sum_{\sigma \in \mathcal{E}} \int_{\sigma}\left(\mu \boldsymbol{n}_{\sigma} \cdot\left(\nabla \boldsymbol{U}^{n}-\left[\nabla \boldsymbol{U}^{n}\right]_{\sigma}\right) \cdot\left[\boldsymbol{u}^{n}-\boldsymbol{U}_{h}^{n}\right]_{\sigma, \mathbf{n}_{\sigma}}\right. \\
& \left.+(\lambda+\mu)\left(\operatorname{div} \boldsymbol{U}^{n}-\left[\operatorname{div} \boldsymbol{U}^{n}\right]_{\sigma}\right)\left[\boldsymbol{u}^{n}-\boldsymbol{U}_{h}^{n}\right]_{\sigma, \mathbf{n}_{\sigma}} \cdot \boldsymbol{n}_{\sigma}\right) \mathrm{d} S ;
\end{aligned}
$$

whence by using the first order Taylor formula applied to functions $x \mapsto \nabla \mathbf{U}^{n}(x)$ to evaluate the differences $\nabla \boldsymbol{U}^{n}-\left[\nabla \boldsymbol{U}^{n}\right]_{\sigma}, \operatorname{div} \boldsymbol{U}^{n}-\left[\operatorname{div} \boldsymbol{U}^{n}\right]_{\sigma}$, and Hölder's inequality,

$$
\begin{aligned}
& \left|I_{2}\right| \leq k h c\left\|\nabla^{2} \boldsymbol{U}\right\|_{L^{\infty}\left(Q_{T} ; \mathbb{R}^{27}\right)} \sum_{n=1}^{m} \sum_{\sigma \in \mathcal{E}} \sqrt{|\sigma|} \sqrt{h}\left(\frac{1}{\sqrt{h}}\left\|\left[\boldsymbol{u}^{n}-\boldsymbol{U}_{h}^{n}\right]_{\sigma, \mathbf{n}_{\sigma}}\right\|_{L^{2}\left(\sigma ; \mathbb{R}^{3}\right)}\right) \\
& \leq k h c\left\|\nabla^{2} \boldsymbol{U}\right\|_{L^{\infty}\left(Q_{T} ; \mathbb{R}^{27}\right)} \sum_{n=1}^{m} \sum_{\sigma \in \mathcal{E}}\left(|\sigma| h+\frac{1}{h}\left\|\left[\boldsymbol{u}^{n}-\boldsymbol{U}_{h}^{n}\right]_{\sigma, \mathbf{n}_{\sigma}}\right\|_{L^{2}\left(\sigma ; \mathbb{R}^{3}\right)}^{2}\right) .
\end{aligned}
$$

Therefore,

$$
\left|\mathcal{R}_{1,1}\right| \leq h c\left(M_{0}, E_{0},\left\|\mathbf{U}, \nabla \boldsymbol{U}, \nabla^{2} \boldsymbol{U}\right\|_{L^{\infty}\left(Q_{T}, \mathbb{R}^{39}\right)}\right),
$$

where we have employed Lemma 9.5 and (4.9) in Corollary 4.1. 
Step 2: $\operatorname{Term} \mathcal{T}_{2}$. Let us now decompose the term $\mathcal{T}_{2}$ as

$\mathcal{T}_{2}=\mathcal{T}_{2,1}+\mathcal{R}_{2,1}$

with $\mathcal{T}_{2,1}=k \sum_{n=1}^{m} \sum_{K \in \mathcal{T}} \int_{K} r^{n-1} \frac{\boldsymbol{U}^{n}-\boldsymbol{U}^{n-1}}{k} \cdot\left(\boldsymbol{u}^{n}-\boldsymbol{U}_{h}^{n}\right) \mathrm{d} x, \quad \mathcal{R}_{2,1}=k \sum_{n=1}^{m} \sum_{K \in \mathcal{T}} \mathcal{R}_{2,1}^{n, K}$,

and $\mathcal{R}_{2,1}^{n, K}=\int_{K}\left(r^{n}-r^{n-1}\right)\left[\partial_{t} \mathbf{U}^{n} \cdot\left(\boldsymbol{u}^{n}-\boldsymbol{U}_{h}^{n}\right) \mathrm{d} x+\int_{K} r^{n-1}\left(\left[\partial_{t} \boldsymbol{U}\right]^{n}-\frac{\boldsymbol{U}^{n}-\boldsymbol{U}^{n-1}}{k}\right) \cdot\left(\boldsymbol{u}^{n}-\boldsymbol{U}_{h}^{n}\right) \mathrm{d} x\right.$.

The remainder $\mathcal{R}_{2,1}^{n, K}$ can be rewritten as follows

$$
\begin{gathered}
\mathcal{R}_{2,1}^{n, K}=\int_{K}\left[\int_{t_{n-1}}^{t_{n}} r(t, \cdot) \mathrm{d} t\right]\left[\partial_{t} \mathbf{U}\right]^{n} \cdot\left(\boldsymbol{u}^{n}-\boldsymbol{U}_{h}^{n}\right) \mathrm{d} x \\
+\frac{1}{k} \int_{K} r^{n-1}\left[\int_{t_{n-1}}^{t_{n}} \int_{s}^{t_{n}} \partial_{t}^{2} \boldsymbol{U}(z, \cdot) \mathrm{d} z \mathrm{~d} s\right] \cdot\left(\boldsymbol{u}^{n}-\boldsymbol{U}_{h}^{n}\right) \mathrm{d} x ;
\end{gathered}
$$

whence,

$$
\begin{gathered}
\left|\mathcal{R}_{2,1}^{n, K}\right| \leq k\left[( \| r \| _ { L ^ { \infty } ( Q _ { T } ) } + \| \partial _ { t } r \| _ { L ^ { \infty } ( Q _ { T } ) } ) \left(\left\|\partial_{t} \boldsymbol{U}\right\|_{L^{\infty}\left(Q_{T} ; \mathbb{R}^{3}\right)}|K|^{5 / 6}\left(\left\|\boldsymbol{u}^{n}\right\|_{L^{6}(K)}+\left\|\boldsymbol{U}_{h}^{n}\right\|_{L^{6}(K)}\right)\right.\right. \\
+\left\|\partial_{t}^{2} \boldsymbol{U}^{n}\right\|_{\left.L^{6 / 5}\left(\Omega ; \mathbb{R}^{3}\right)\right)}\left(\left\|\boldsymbol{u}^{n}\right\|_{L^{6}(K)}+\left\|\boldsymbol{U}_{h}^{n}\right\|_{L^{6}(K)}\right) .
\end{gathered}
$$

Consequently, by the same token as in (6.14) or (6.22),

$$
\left|\mathcal{R}_{2,1}\right| \leq k c\left(M_{0}, E_{0}, \bar{r},\left\|\left(\partial_{t} r, \boldsymbol{U}, \partial_{t} \boldsymbol{U}, \nabla \boldsymbol{U},\right)\right\|_{L^{\infty}\left(Q_{T} ; \mathbb{R}^{16}\right)},\left\|\partial_{t}^{2} \boldsymbol{U}\right\|_{L^{2}\left(0, T ; L^{6 / 5}\left(\Omega ; \mathbb{R}^{3}\right)\right)}\right),
$$

where we have used the Hölder and Young inequalities, the estimates (9.21), (9.24), (9.29), (9.30), and to the energy bound (4.9) from Corollary 4.1,

Step 2a: $\operatorname{Term} \mathcal{T}_{2,1}$. We decompose the term $\mathcal{T}_{2,1}$ as

$$
\begin{aligned}
& \mathcal{T}_{2,1}=\mathcal{T}_{2,2}+\mathcal{R}_{2,2}, \\
& \text { with } \mathcal{T}_{2,2}=k \sum_{n=1}^{m} \sum_{K \in \mathcal{T}} \int_{K} r_{K}^{n-1} \frac{\boldsymbol{U}^{n}-\boldsymbol{U}^{n-1}}{k} \cdot\left(\boldsymbol{u}^{n}-\boldsymbol{U}_{h}^{n}\right) \mathrm{d} x, \mathcal{R}_{2,2}=k \sum_{n=1}^{m} \sum_{K \in \mathcal{T}} \mathcal{R}_{2,2}^{n, K}, \\
& \text { and } \mathcal{R}_{2,2}^{n, K}=\int_{K}\left(r^{n-1}-r_{K}^{n-1}\right) \frac{\boldsymbol{U}^{n}-\boldsymbol{U}^{n-1}}{k} \cdot\left(\boldsymbol{u}^{n}-\boldsymbol{U}_{h}^{n}\right) \mathrm{d} x ;
\end{aligned}
$$

therefore,

$$
\left|\mathcal{R}_{2,2}^{n}\right|=\left|\sum_{K \in \mathcal{T}} \mathcal{R}_{2,2}^{n, K}\right| \leq h c\|\nabla r\|_{L^{\infty}\left(Q_{T} ; \mathbb{R}^{3}\right)}\left\|\partial_{t} \boldsymbol{U}\right\|_{L^{\infty}\left(Q_{T} ; \mathbb{R}^{3}\right)}\left\|\boldsymbol{u}^{n}-\boldsymbol{U}_{h}^{n}\right\|_{L^{6}\left(\Omega ; \mathbb{R}^{3}\right)} .
$$

Consequently, by virtue of formula (4.10) in Corollary 4.1 and estimates (9.30), (9.25),

$$
\left|\mathcal{R}_{2,2}\right| \leq h c\left(M_{0}, E_{0},\left\|\left(\nabla r, \boldsymbol{U}, \partial_{t} \boldsymbol{U}, \nabla \boldsymbol{U}\right)\right\|_{L^{\infty}\left(Q_{T} ; \mathbb{R}^{18}\right)}\right) .
$$

Step 2b: Term $\mathcal{T}_{2,2}$. We decompose the term $\mathcal{T}_{2,2}$ as

$$
\begin{aligned}
& \mathcal{T}_{2,2}=\mathcal{T}_{2,3}+\mathcal{R}_{2,3}, \\
& \text { with } \mathcal{T}_{2,3}=k \sum_{n=1}^{m} \sum_{K \in \mathcal{T}} \int_{K} r_{K}^{n-1} \frac{\boldsymbol{U}_{h, K}^{n}-\boldsymbol{U}_{h, K}^{n-1}}{k} \cdot\left(\boldsymbol{u}^{n}-\boldsymbol{U}_{h}^{n}\right) \mathrm{d} x, \mathcal{R}_{2,3}=k \sum_{n=1}^{m} \sum_{K \in \mathcal{T}} \mathcal{R}_{2,3}^{n, K}, \\
& \text { and } \mathcal{R}_{2,3}^{n, K}=\int_{K} r_{K}^{n-1}\left(\frac{\boldsymbol{U}^{n}-\boldsymbol{U}^{n-1}}{k}-\left[\frac{\boldsymbol{U}^{n}-\boldsymbol{U}^{n-1}}{k}\right]_{h}\right) \cdot\left(\boldsymbol{u}^{n}-\boldsymbol{U}_{h}^{n}\right) \mathrm{d} x \\
& +\int_{K} r_{K}^{n-1}\left(\left[\frac{\boldsymbol{U}^{n}-\boldsymbol{U}^{n-1}}{k}\right]_{h}-\left[\frac{\boldsymbol{U}^{n}-\boldsymbol{U}^{n-1}}{k}\right]_{h, K}\right) \cdot\left(\boldsymbol{u}^{n}-\boldsymbol{U}_{h}^{n}\right) \mathrm{d} x=I_{1}^{K}+I_{2}^{K} .
\end{aligned}
$$


We have

$$
\begin{gathered}
\left|I_{2}^{K}\right|=\frac{1}{k} r_{K}^{n-1}\left|\int_{K}\left(\left[\int_{t_{n-1}}^{t_{n}} \partial_{t} \boldsymbol{U}(z) \mathrm{d} z\right]_{h}-\left[\int_{t_{n-1}}^{t_{n}} \partial_{t} \boldsymbol{U}(z) \mathrm{d} z\right]_{h, K}\right) \cdot\left(\boldsymbol{u}^{n}-\boldsymbol{U}_{h}^{n}\right) \mathrm{d} x\right| \\
\leq \frac{h}{k} r_{K}^{n-1} \int_{t_{n-1}}^{t_{n}}\left\|\nabla_{x}\left[\partial_{t} \boldsymbol{U}(z)\right]_{h}\right\|_{L^{6 / 5}\left(K ; \mathbb{R}^{3}\right)}\left\|\boldsymbol{u}^{n}-\boldsymbol{U}_{h}^{n}\right\|_{L^{6}\left(K ; \mathbb{R}^{3}\right)}
\end{gathered}
$$

where we have used the Fubini theorem, Hölder's inequality and (9.1), (9.22) $s=1$. Further, employing the Sobolev inequality on Crouzeix-Raviart space (9.30), Hölder's and Young's inequalities and estimate $(9.22)_{s=1}$, we get

$$
\begin{gathered}
\sum_{K \in \mathcal{T}}\left|I_{2}^{K}\right| \leq \frac{h}{k} r_{K}^{n-1}\left\|\boldsymbol{u}^{n}-\boldsymbol{U}_{h}^{n}\right\|_{L^{6}\left(\Omega ; \mathbb{R}^{3}\right)} \int_{t_{n-1}}^{t_{n}}\left\|\nabla_{x} \partial_{t} \boldsymbol{U}(z)\right\|_{L^{6 / 5}\left(\Omega ; \mathbb{R}^{3}\right)} \mathrm{d} z \\
\quad \leq c r_{K}^{n-1}\left(h\left|\boldsymbol{u}^{n}-\boldsymbol{U}_{h}^{n}\right|_{V_{h}^{2}\left(\Omega ; \mathbb{R}^{3}\right)}^{2}+\frac{h}{k} \int_{t_{n-1}}^{t_{n}}\left\|\nabla_{x} \partial_{t} \boldsymbol{U}(z)\right\|_{L^{6 / 5}\left(\Omega ; \mathbb{R}^{3}\right)}^{2}\right)
\end{gathered}
$$

We reserve the similar treatment to the term $I_{1}^{K}$. Resuming these calculations we get by using Corollary 4.1

$$
\left|\mathcal{R}_{2,3}\right| \leq h c\left(M_{0}, E_{0},\left\|\left(r, \boldsymbol{U}, \nabla \boldsymbol{U}, \partial_{t} \boldsymbol{U}\right)\right\|_{L^{\infty}\left(Q_{T} ; \mathbb{R}^{16}\right)},\left\|\partial_{t} \nabla \boldsymbol{U}\right\|_{L^{2}\left(0, T ; L^{6 / 5}\left(\Omega ; \mathbb{R}^{9}\right)\right)}\right) .
$$

Step 2c: Term $\mathcal{T}_{2,3}$. We rewrite this term in the form

$$
\begin{aligned}
& \mathcal{T}_{2,3}=\mathcal{T}_{2,4}+\mathcal{R}_{2,4}, \mathcal{R}_{2,4}=k \sum_{n=1}^{m} \sum_{K \in \mathcal{T}} \mathcal{R}_{2,4}^{n, K}, \\
& \text { with } \mathcal{T}_{2,4}=k \sum_{n=1}^{m} \sum_{K \in \mathcal{T}} \int_{K} r_{K}^{n-1} \frac{\boldsymbol{U}_{h, K}^{n}-\boldsymbol{U}_{h, K}^{n-1}}{k} \cdot\left(\boldsymbol{u}_{K}^{n}-\boldsymbol{U}_{h, K}^{n}\right) \mathrm{d} x, \\
& \text { and } \mathcal{R}_{2,4}^{n, K}=\int_{K} r_{K}^{n-1} \frac{\boldsymbol{U}_{h, K}^{n}-\boldsymbol{U}_{h, K}^{n-1}}{k} \cdot\left(\left(\boldsymbol{u}^{n}-\boldsymbol{u}_{K}^{n}\right)-\left(\boldsymbol{U}_{h}^{n}-\boldsymbol{U}_{h, K}^{n}\right)\right) \mathrm{d} x .
\end{aligned}
$$

First we write, as in (6.3),

$$
\begin{gathered}
\left|\frac{\left[\boldsymbol{U}^{n}-\boldsymbol{U}^{n-1}\right]_{h, K}}{k}\right|=\left|\frac{1}{|K|} \int_{K}\left[\frac{1}{k}\left[\int_{t_{n-1}}^{t_{n}} \partial_{t} \mathbf{U}(z, x) \mathrm{d} z\right]_{h}\right] \mathrm{d} x\right| \\
=\left|\frac{1}{|K|} \int_{K}\left[\frac{1}{k} \int_{t_{n-1}}^{t_{n}}\left[\partial_{t} \mathbf{U}(z)\right]_{h}(x) \mathrm{d} z\right] \mathrm{d} x\right| \leq\left\|\left[\partial_{t} \mathbf{U}\right]_{h}\right\|_{L^{\infty}\left(0, T ; L^{\infty}\left(\Omega ; \mathbb{R}^{3}\right)\right)} \leq\left\|\partial_{t} \mathbf{U}\right\|_{L^{\infty}\left(0, T ; L^{\infty}\left(\Omega ; \mathbb{R}^{3}\right)\right)},
\end{gathered}
$$

Next we evaluate $\boldsymbol{u}^{n}-\boldsymbol{u}_{K}^{n}$ employing $(9.1)_{p=2}$, and $\boldsymbol{U}_{h}^{n}-\boldsymbol{U}_{h, K}^{n}$ by using (9.1) $)_{p=\infty},(9.22)_{s=1}$. Finally we employ the Hölder inequality to get

$$
\left.\left|\mathcal{R}_{2,4}\right| \leq h c\left(M_{0}, E_{0}, \bar{r},\left\|\left(\partial_{t} \boldsymbol{U}, \boldsymbol{U}, \nabla \boldsymbol{U}\right)\right\|_{L^{\infty}\left(Q_{T} ; \mathbb{R}^{15}\right)}, \| \partial_{t} \nabla \boldsymbol{U}\right) \|_{L^{2}\left(0, T ; L^{6 / 5}\left(\Omega ; \mathbb{R}^{9}\right)\right)}\right) .
$$

Step 3: $\operatorname{Term} \mathcal{T}_{3}$. Let us first decompose $\mathcal{T}_{3}$ as

$$
\mathcal{T}_{3}=\mathcal{T}_{3,1}+\mathcal{R}_{3,1},
$$

with $\mathcal{T}_{3,1}=k \sum_{n=1}^{m} \sum_{K \in \mathcal{T}} \int_{K} r_{K}^{n} \boldsymbol{U}_{h, K}^{n} \cdot \nabla \boldsymbol{U}^{n} \cdot\left(\boldsymbol{u}_{K}^{n}-\boldsymbol{U}_{h, K}^{n}\right) \mathrm{d} x, \quad \mathcal{R}_{3,1}=k \sum_{n=1}^{m} \sum_{K \in \mathcal{T}} \mathcal{R}_{3,1}^{n, K}$,

and $\mathcal{R}_{3,1}^{n, K}=\int_{K}\left(r^{n}-r_{K}^{n}\right) \boldsymbol{U}^{n} \cdot \nabla \boldsymbol{U}^{n} \cdot\left(\boldsymbol{u}^{n}-\boldsymbol{U}_{h}^{n}\right) \mathrm{d} x+\int_{K} r_{K}^{n}\left(\boldsymbol{U}^{n}-\boldsymbol{U}_{h}^{n}\right) \cdot \nabla \boldsymbol{U}^{n} \cdot\left(\boldsymbol{u}^{n}-\boldsymbol{U}_{h}^{n}\right) \mathrm{d} x$

$$
+\int_{K} r_{K}^{n}\left(\boldsymbol{U}_{h}^{n}-\boldsymbol{U}_{h, K}^{n}\right) \cdot \nabla \boldsymbol{U}^{n} \cdot\left(\boldsymbol{u}^{n}-\boldsymbol{U}_{h}^{n}\right) \mathrm{d} x+\int_{K} r_{K}^{n} \boldsymbol{U}_{h, K}^{n} \cdot \nabla \boldsymbol{U}^{n} \cdot\left(\boldsymbol{u}^{n}-\boldsymbol{u}_{K}^{n}-\left(\boldsymbol{U}_{h}^{n}-\boldsymbol{U}_{h, K}^{n}\right)\right) \mathrm{d} x .
$$


We find that

$$
\begin{array}{r}
\left|\mathcal{R}_{3,1}^{n, K}\right| \leq h\left[|K|^{1 / 2}\left(\left\|\boldsymbol{u}^{n}\right\|_{L^{2}\left(K ; \mathbb{R}^{3}\right)}+\left\|\boldsymbol{U}_{h}^{n}\right\|_{L^{2}\left(K ; \mathbb{R}^{3}\right)}\right)+|K|^{1 / 2}\left(\left\|\nabla \boldsymbol{u}^{n}\right\|_{L^{2}\left(K ; \mathbb{R}^{3}\right)}+\left\|\nabla \boldsymbol{U}_{h}^{n}\right\|_{L^{2}\left(K ; \mathbb{R}^{3}\right)}\right)\right] \\
\times\left(\|r\|_{L^{\infty}\left(Q_{T}\right)}+\|\nabla r\|_{L^{\infty}\left(Q_{T} ; \mathbb{R}^{3}\right)}\right)\left(\|\boldsymbol{U}\|_{L^{\infty}\left(Q_{T} ; \mathbb{R}^{3}\right)}+\|\nabla \boldsymbol{U}\|_{L^{\infty}\left(Q_{T} ; \mathbb{R}^{9}\right)}\right)^{2},
\end{array}
$$

where we have used several times Hölder's inequality and the standard first order Taylor formula ( to evaluate $r^{n}-r_{K}^{n}$ ), along with the estimates (9.21) (to evaluate $\boldsymbol{U}^{n}-\boldsymbol{U}_{h}^{n}$ ), (9.1), (9.22) $)_{s=1}$ (to evaluate $\boldsymbol{U}_{h}^{n}-\boldsymbol{U}_{h, K}^{n}$ ), (9.1) (to evaluate $\boldsymbol{u}^{n}-\boldsymbol{u}_{K}^{n}$ ).

Consequently, using again $\left({ }^{9.22}\right)_{s=1}$ (to estimate $\left.\left\|\nabla \boldsymbol{U}_{h}^{n}\right\|_{L^{2}\left(K ; \mathbb{R}^{3}\right)}\right)$, the definition of $|\cdot|_{V_{h}^{2}(\Omega)}$ norm, the Sobolev inequality (9.30) and the energy bound (4.9) from Corollary 4.1, we conclude that

$$
\left|\mathcal{R}_{3,1}\right| \leq h c\left(M_{0}, E_{0}, \bar{r},\|(\nabla r, \boldsymbol{U}, \nabla \boldsymbol{U})\|_{L^{\infty}\left(Q_{T} ; \mathbb{R}^{15}\right)}\right) .
$$

Now we shall deal wit term $\mathcal{T}_{3,1}$. Integrating by parts, we get:

$$
\begin{aligned}
\int_{K} r_{K}^{n} \boldsymbol{U}_{h, K}^{n} \cdot \nabla \boldsymbol{U}^{n} \cdot\left(\boldsymbol{u}_{K}^{n}-\boldsymbol{U}_{h, K}^{n}\right) \mathrm{d} x & =\sum_{\sigma \in \mathcal{E}(K)}|\sigma| r_{K}^{n}\left[\boldsymbol{U}_{h, K}^{n} \cdot \mathbf{n}_{\sigma, K}\right] \boldsymbol{U}_{\sigma}^{n} \cdot\left(\boldsymbol{u}_{K}^{n}-\boldsymbol{U}_{h, K}^{n}\right) \\
& =\sum_{\sigma \in \mathcal{E}(K)}|\sigma| r_{K}^{n}\left[\boldsymbol{U}_{h, K}^{n} \cdot \mathbf{n}_{\sigma, K}\right]\left(\boldsymbol{U}_{\sigma}^{n}-\boldsymbol{U}_{h, K}^{n}\right) \cdot\left(\boldsymbol{u}_{K}^{n}-\boldsymbol{U}_{h, K}^{n}\right),
\end{aligned}
$$

thanks to the the fact that $\sum_{\sigma \in \mathcal{E}(K)} \int_{\sigma} \boldsymbol{U}_{h, K}^{n} \cdot \mathbf{n}_{\sigma, K} \mathrm{~d} S=0$.

Next we write

$$
\begin{gathered}
\mathcal{T}_{3,1}=\mathcal{T}_{3,2}+\mathcal{R}_{3,2}, \quad \mathcal{R}_{3,2}=k \sum_{n=1}^{m} \mathcal{R}_{3,2}^{n}, \\
\mathcal{T}_{3,2}=k \sum_{n=1}^{m} \sum_{K \in \mathcal{T}} \sum_{\sigma \in \mathcal{E}(K)}|\sigma| \hat{r}_{\sigma}^{n, \text { up }}\left[\hat{\boldsymbol{U}}_{h, \sigma}^{n, \mathrm{up}} \cdot \mathbf{n}_{\sigma, K}\right]\left(\boldsymbol{U}_{\sigma}^{n}-\boldsymbol{U}_{h, K}^{n}\right) \cdot\left(\hat{\boldsymbol{u}}_{\sigma}^{n, \mathrm{up}}-\hat{\boldsymbol{U}}_{h, \sigma}^{n, \mathrm{up}}\right), \\
\text { and } \mathcal{R}_{3,2}^{n}=\sum_{K \in \mathcal{T}} \sum_{\sigma \in \mathcal{E}(K)}|\sigma|\left(r_{K}^{n}-\hat{r}_{\sigma}^{n, \mathrm{up}}\right)\left[\boldsymbol{U}_{h, K}^{n} \cdot \mathbf{n}_{\sigma, K}\right]\left(\boldsymbol{U}_{\sigma}^{n}-\boldsymbol{U}_{h, K}^{n}\right) \cdot\left(\boldsymbol{u}_{K}^{n}-\boldsymbol{U}_{h, K}^{n}\right) \\
+\sum_{K \in \mathcal{T}} \sum_{\sigma \in \mathcal{E}(K)}|\sigma| \hat{r}_{\sigma}^{n, \mathrm{up}}\left[\left(\boldsymbol{U}_{h, K}^{n}-\hat{\boldsymbol{U}}_{h, \sigma}^{n, \text { up }}\right) \cdot \mathbf{n}_{\sigma, K}\right]\left(\boldsymbol{U}_{\sigma}^{n}-\boldsymbol{U}_{h, K}^{n}\right) \cdot\left(\boldsymbol{u}_{K}^{n}-\boldsymbol{U}_{h, K}^{n}\right) \\
+\sum_{K \in \mathcal{T}} \sum_{\sigma \in \mathcal{E}(K)}|\sigma| \hat{r}_{\sigma}^{n, \mathrm{up}}\left[\hat{\boldsymbol{U}}_{h, \sigma}^{n, \mathrm{up}} \cdot \mathbf{n}_{\sigma, K}\right]\left(\boldsymbol{U}_{\sigma}^{n}-\boldsymbol{U}_{h, K}^{n}\right) \cdot\left(\left(\boldsymbol{u}_{K}^{n}-\hat{\boldsymbol{u}}_{h, \sigma}^{n, \mathrm{up}}\right)-\left(\boldsymbol{U}_{h, K}^{n}-\hat{\boldsymbol{U}}_{h, \sigma}^{n, \mathrm{up}}\right)\right) .
\end{gathered}
$$

We may use several times the Taylor formula (in order to estimate $r_{K}^{n}-\hat{r}_{\sigma}^{n, \text { up }}, \mathbf{U}_{\sigma}^{n}-\mathbf{U}_{h, K}^{n}, \mathbf{U}_{h, K}^{n}-\hat{\mathbf{U}}_{h, \sigma}^{n, \text { up }}$ ) to get the bound

$$
\begin{gathered}
\left|\mathcal{R}_{3,2}^{n}\right| \leq h c\|r\|_{W^{1, \infty}(\Omega)}\left(1+\|\boldsymbol{U}\|_{W^{1, \infty}\left(\Omega ; \mathbb{R}^{3}\right)}\right)^{3} \sum_{K \in \mathcal{T}} h|\sigma|\left|\boldsymbol{u}_{K}^{n}\right| \\
+c\|r\|_{W^{1, \infty}(\Omega)}\left(1+\|\boldsymbol{U}\|_{W^{1, \infty}\left(\Omega ; \mathbb{R}^{3}\right)}\right)^{2} \sum_{K \in \mathcal{T}} \sum_{\sigma \in \mathcal{E}(K)} h|\sigma|\left|\boldsymbol{u}_{K}^{n}-\boldsymbol{u}_{\sigma}^{n}\right|
\end{gathered}
$$

where by virtue of Hölder's inequality, (9.16), (9.31), (9.18) (9.19),

$$
\sum_{K \in \mathcal{T}} h|\sigma|\left|\boldsymbol{u}_{K}^{n}\right| \leq c\left(\sum_{\sigma \in \mathcal{T}} h|\sigma|\left|\boldsymbol{u}_{K}^{n}\right|^{6}\right)^{1 / 6} \leq c\left[\left(\sum_{K \in \mathcal{T}}\left\|\boldsymbol{u}^{n}-\boldsymbol{u}_{K}^{n}\right\|_{L^{6}\left(K ; \mathbb{R}^{3}\right)}^{6}\right)^{1 / 6}\right.
$$




$$
\begin{gathered}
\left.+\left(\sum_{K \in \mathcal{T}}\left\|\boldsymbol{u}^{n}\right\|_{L^{6}\left(K ; \mathbb{R}^{3}\right)}^{6}\right)^{1 / 6}\right] \leq c\left(\sum_{K \in \mathcal{T}}\left\|\nabla \boldsymbol{u}_{n}\right\|_{L^{2}\left(K ; \mathbb{R}^{9}\right)}^{2}\right)^{1 / 2}, \\
\sum_{K \in \mathcal{T}} \sum_{\sigma \in \mathcal{E}(K)} h\left|\sigma \| \boldsymbol{u}_{K}^{n}-\boldsymbol{u}_{\sigma}^{n}\right| \leq c\left[\left(\sum_{K \in \mathcal{T}}\left\|\boldsymbol{u}^{n}-\boldsymbol{u}_{K}^{n}\right\|_{L^{2}\left(K ; \mathbb{R}^{3}\right)}^{2}\right)^{1 / 2}\right. \\
\left.+\left(\sum_{K \in \mathcal{T}} \sum_{\sigma \in \mathcal{E}(K)}\left\|\boldsymbol{u}^{n}-\boldsymbol{u}_{\sigma}^{n}\right\|_{L^{2}\left(K ; \mathbb{R}^{3}\right)}^{2}\right)^{1 / 2}\right] \leq h c\left(\sum_{K \in \mathcal{T}}\left\|\nabla \boldsymbol{u}_{n}\right\|_{L^{2}\left(K ; \mathbb{R}^{9}\right)}^{2}\right)^{1 / 2},
\end{gathered}
$$

Consequently, we may use (4.9) to conclude

$$
\left|\mathcal{R}_{3,2}\right| \leq h c\left(M_{0}, E_{0}, \bar{r},\|\nabla r, \boldsymbol{U}, \nabla \boldsymbol{U}\|_{L^{\infty}\left(Q_{T} ; \mathbb{R}^{15}\right)}\right) .
$$

Step 4: Terms $\mathcal{T}_{4}$ and $\mathcal{T}_{5}$. We decompose $\mathcal{T}_{4}$ as

$\mathcal{T}_{4}=\mathcal{T}_{4,1}+\mathcal{R}_{4,1}$,

with $\mathcal{T}_{4,1}=k \sum_{n=1}^{m} \sum_{K \in \mathcal{T}} \int_{K} p^{\prime}\left(r_{K}^{n}\right) \boldsymbol{u}^{n} \cdot \nabla r^{n} \mathrm{~d} x, \quad \mathcal{R}_{4,1}=k \sum_{n=1}^{m} \sum_{K \in \mathcal{T}} \int_{K}\left(p^{\prime}\left(r^{n}\right)-p^{\prime}\left(r_{K}^{n}\right)\right) \boldsymbol{u}^{n} \cdot \nabla r^{n} \mathrm{~d} x$

whence

$$
\left|\mathcal{R}_{4,1}\right| \leq h c\left(M_{0}, E_{0}, \underline{r}, \bar{r},\left|p^{\prime}\right|_{C^{1}([\underline{r}, \bar{r}])},\|\nabla r\|_{L^{\infty}\left(Q_{T} ; \mathbb{R}^{3}\right)}\right) .
$$

Employing integration by parts, we infer

$$
\begin{aligned}
& \mathcal{T}_{5}=\mathcal{T}_{5,1}+\mathcal{R}_{5,1}, \text { with } \mathcal{T}_{5,1}=-k \sum_{n=1}^{m} \sum_{K \in \mathcal{T}} \int_{K} \nabla p\left(r^{n}\right) \cdot \boldsymbol{U}^{n} \mathrm{~d} x, \\
& \mathcal{R}_{5,1}=k \sum_{n=1}^{m} \sum_{K \in \mathcal{T}} \int_{K} \nabla p\left(r^{n}\right) \cdot\left(\boldsymbol{U}^{n}-\boldsymbol{U}_{h}^{n}\right) \mathrm{d} x
\end{aligned}
$$

and

$$
\left|\mathcal{R}_{5,1}\right| \leq h c\left(\bar{r},\left|p^{\prime}\right|_{C([r, \bar{r}])},\|\nabla r, \nabla \boldsymbol{U}\|_{L^{\infty}\left(Q_{T} ; \mathbb{R}^{12}\right)}\right) .
$$

Integrating by parts, we obtain

$$
\mathcal{T}_{5,1}=k \sum_{n=1}^{m} \sum_{K \in \mathcal{T}} \int_{K} p\left(r^{n}\right) \operatorname{div} \boldsymbol{U}^{n} \mathrm{~d} x
$$

whence

$$
\begin{aligned}
\mathcal{T}_{5,1} & =\mathcal{T}_{5,2}+\mathcal{R}_{5,2}, \text { with } \mathcal{T}_{5,2}=k \sum_{n=1}^{m} \sum_{K \in \mathcal{T}} \int_{K} p\left(r_{K}^{n}\right) \operatorname{div} \boldsymbol{U}^{n} \mathrm{~d} x, \\
\mathcal{R}_{5,2} & =-k \sum_{n=1}^{m} \sum_{K \in \mathcal{T}} \int_{K}\left(p\left(r_{K}^{n}\right)-p\left(r^{n}\right)\right) \operatorname{div} \boldsymbol{U}^{n} \mathrm{~d} x
\end{aligned}
$$

and

$$
\left|\mathcal{R}_{5,2}\right| \leq h c\left(\bar{r},\left|p^{\prime}\right|_{[\underline{r}, \bar{r}]},\|\nabla r, \nabla \boldsymbol{U}\|_{L^{\infty}\left(Q_{T} ; \mathbb{R}^{12}\right)}\right) .
$$

Gathering the formulae (7.7), (17.12), (77.15), (17.17), (7.19) and estimates for the residual terms (7.8), (7.9 (7.13), (7.14 (7.16), (7.18), (7.20), (7.21) concludes the proof of Lemma 7.1. 


\section{8 - End of the proof of the error estimate (Theorem 3.1)}

In this Section we put together the relative energy inequality (6.1) and the identity (7.1) derived in the previous section. The final inequality resulting from this manipulation is formulated in the following lemma.

Lemma 8.1. Under assumptions of Theorem 3.1 there exists a positive number

$$
c=c\left(M_{0}, E_{0}, \underline{r}, \bar{r},\left|p^{\prime}\right|_{C^{1}([\underline{r}, \bar{r}])},\left\|\left(\nabla r, \partial_{t} r, \partial_{t} \nabla r, \partial_{t}^{2} r, \boldsymbol{U}, \nabla \boldsymbol{U}, \nabla^{2} \boldsymbol{U}, \partial_{t} \boldsymbol{U}, \partial_{t} \nabla \boldsymbol{U}\right)\right\|_{L^{\infty}\left(Q_{T} ; \mathbb{R}^{65}\right)}\right)
$$

(depending tacitly also on $T, \theta_{0}, \gamma$, $\operatorname{diam}(\Omega),|\Omega|$ ), such that for all $m=1, \ldots, N$, there holds:

$$
\begin{gathered}
\mathcal{E}\left(\varrho^{m}, \boldsymbol{u}^{m} \mid r^{m}, \boldsymbol{u}^{m}\right)+k \frac{\mu}{2} \sum_{n=1}^{m} \sum_{K \in \mathcal{T}} \int_{K}\left|\nabla_{x}\left(\mathbf{u}^{n}-\mathbf{U}_{h}^{n}\right)\right|^{2} \mathrm{dx} \\
\leq c\left[h^{A}+\sqrt{k}+\mathcal{E}\left(\varrho^{0}, \boldsymbol{u}^{0} \mid r^{0}, \boldsymbol{U}^{0}\right)\right]+c k \sum_{n=1}^{m} \mathcal{E}\left(\varrho^{n}, \boldsymbol{u}^{n} \mid r^{n}, \boldsymbol{u}^{n}\right),
\end{gathered}
$$

where $A$ is defined in (6.2).

Proof. Gathering the formulae (6.1) and (7.1), one gets

$$
\begin{gathered}
\mathcal{E}\left(\varrho^{m}, \boldsymbol{u}^{m} \mid r^{m}, U^{m}\right)-\mathcal{E}\left(\varrho^{0}, \boldsymbol{u}^{0} \mid r(0), \boldsymbol{U}(0)\right)+\mu k \sum_{n=1}^{m}\left|\boldsymbol{u}^{n}-\boldsymbol{U}_{h}^{n}\right|_{V_{h}^{2}\left(\Omega ; \mathbb{R}^{3}\right)}^{2} \\
\leq \mathcal{P}_{1}+\mathcal{P}_{2}+\mathcal{P}_{3}+\mathcal{Q}
\end{gathered}
$$

where

$$
\begin{aligned}
& \mathcal{P}_{1}=k \sum_{n=1}^{m} \sum_{K \in \mathcal{T}}|K|\left(\varrho_{K}^{n-1}-r_{K}^{n-1}\right) \frac{\boldsymbol{U}_{h, K}^{n}-\boldsymbol{U}_{h, K}^{n-1}}{k} \cdot\left(\boldsymbol{U}_{h, K}^{n}-\boldsymbol{u}_{K}^{n}\right), \\
& \mathcal{P}_{2}=k \sum_{n=1}^{m} \sum_{K \in \mathcal{T}} \sum_{\sigma=K \mid L \in \mathcal{E}(K)}|\sigma|\left(\varrho_{\sigma}^{n, \mathrm{up}}-\hat{r}_{\sigma}^{n, \text { up }}\right)\left(\hat{\boldsymbol{U}}_{h, \sigma}^{n, \mathrm{up}}-\hat{\boldsymbol{u}}_{\sigma}^{n, \mathrm{up}}\right) \cdot\left(\boldsymbol{U}_{\sigma}^{n}-\boldsymbol{U}_{h, K}^{n}\right) \hat{\boldsymbol{U}}_{h, \sigma}^{n, \mathrm{up}} \cdot \boldsymbol{n}_{\sigma, K}, \\
& \mathcal{P}_{3}=k \sum_{n=1}^{m} \sum_{K \in \mathcal{T}} \int_{K}\left(p\left(r_{K}^{n}\right)-p\left(\varrho_{K}^{n}\right)\right) \operatorname{div} \boldsymbol{U}^{n} \mathrm{~d} x \\
& \quad+k \sum_{n=1}^{m} \sum_{K \in \mathcal{T}}\left[\int_{K} \frac{r_{K}^{n}-\varrho_{K}^{n}}{r_{K}^{n}} p^{\prime}\left(r_{K}^{n}\right) \boldsymbol{u}^{n} \cdot \nabla r^{n} \mathrm{~d} x+\int_{K} \frac{r_{K}^{n}-\varrho_{K}^{n}}{r_{K}^{n}} p^{\prime}\left(r_{K}^{n}\right)\left[\partial_{t} r\right]^{n} \mathrm{~d} x\right], \\
& \mathcal{Q}=\mathcal{R}_{h, k}^{m}+R_{h, k}^{m}+G^{m} .
\end{aligned}
$$

Now, we estimate conveniently the terms $\mathcal{P}_{1}, \mathcal{P}_{2}, \mathcal{P}_{3}$ in three steps.

Step 1: Term $\mathcal{P}_{1}$. We have

$$
\left|\boldsymbol{U}_{h, K}^{n}-\boldsymbol{U}_{h, K}^{n-1}\right| \leq \int_{t_{n-1}}^{t_{n}}\left\|\left[\partial_{t} \boldsymbol{U}(z)\right]_{h}\right\|_{L^{\infty}\left(K ; \mathbb{R}^{9}\right)} \mathrm{dz} \leq c \int_{t_{n-1}}^{t_{n}}\left\|\partial_{t} \boldsymbol{U}(z)\right\|_{L^{\infty}\left(K ; \mathbb{R}^{9}\right)} \mathrm{dz},
$$

where we have used (9.20).

According to Lemma 7.2 ,

$$
|\varrho-r|^{\gamma} 1_{R_{+} \backslash[\underline{r} / 2,2 \bar{r}]}(\varrho) \leq c(p) E^{p}(\varrho \mid r)
$$

with any $p \geq 1$. In particular,

$$
|\varrho-r|^{6 / 5} 1_{R_{+} \backslash[\underline{r} / 2,2 \bar{r}]}(\varrho) \leq c E(\varrho \mid r)
$$


provided $\gamma \geq 6 / 5$.

We get by using the Hölder inequality,

$$
\begin{gathered}
\left|\sum_{K \in \mathcal{T}}\right| K\left|\left(\varrho_{K}^{n-1}-r_{K}^{n-1}\right) \frac{\boldsymbol{U}_{h, K}^{n}-\boldsymbol{U}_{h, K}^{n-1}}{k} \cdot\left(\boldsymbol{U}_{h, K}^{n}-\boldsymbol{u}_{K}^{n}\right)\right| \leq c\left(\left\|\partial_{t} \boldsymbol{U}\right\|_{L^{\infty}\left(Q_{T} ; \mathbb{R}^{3}\right)}+\left\|\partial_{t} \nabla \boldsymbol{U}\right\|_{L^{\infty}\left(Q_{T} ; \mathbb{R}^{9}\right)}\right) \times \\
{\left[\left(\sum_{K \in \mathcal{T}}|K|\left|\varrho_{K}^{n-1}-r_{K}^{n-1}\right|^{2} 1_{[\underline{r} / 2,2 \bar{r}]}\left(\varrho_{K}\right)\right)^{1 / 2}+\left(\sum_{K \in \mathcal{T}}\left|K \| \varrho_{K}^{n-1}-r_{K}^{n-1}\right|^{6 / 5} 1_{R_{+} \backslash[\underline{r} / 2,2 \bar{r}]}\left(\varrho_{K}\right)\right)^{5 / 6}\right] \times} \\
\left(\sum_{K \in \mathcal{T}}|K|\left|\boldsymbol{U}_{h, K}^{n}-\boldsymbol{u}_{K}^{n}\right|^{6}\right)^{1 / 6} \leq c\left(\left\|\left(\partial_{t} \boldsymbol{U}^{1 / 6} \partial_{t} \nabla \boldsymbol{U}\right)\right\|_{L^{\infty}\left(Q_{T} ; \mathbb{R}^{12}\right)}\right)\left(\mathcal{E}^{1 / 2}\left(\varrho^{n-1}, \boldsymbol{u}^{n-1} \mid r^{n-1}, \boldsymbol{U}^{n-1}\right)\right. \\
\left.+\mathcal{E}^{5 / 6}\left(\varrho^{n-1}, \boldsymbol{u}^{n-1} \mid r^{n-1}, \boldsymbol{U}^{n-1}\right)\right)\left(\sum_{K \in \mathcal{T}}\left\|\boldsymbol{U}_{h, K}^{n}-\boldsymbol{u}_{K}^{n}\right\|_{L^{6}\left(K ; \mathbb{R}^{3}\right)}^{6}\right)^{1 / 6},
\end{gathered}
$$

where we have used (8.2) and estimate (4.13) to obtain the last line.

Now, by the Minkowski inequality,

$$
\begin{gathered}
\left(\sum_{K \in \mathcal{T}}\left\|\boldsymbol{U}_{h, K}^{n}-\boldsymbol{u}_{K}^{n}\right\|_{L^{6}\left(K ; \mathbb{R}^{3}\right)}^{6}\right)^{1 / 6} \leq\left(\sum_{K \in \mathcal{T}}\left\|\left(\boldsymbol{U}_{h, K}^{n}-\boldsymbol{u}_{K}^{n}\right)-\left(\boldsymbol{U}_{h}^{n}-\boldsymbol{u}^{n}\right)\right\|_{L^{6}\left(K ; \mathbb{R}^{3}\right)}^{6}\right)^{1 / 6} \\
+\left\|\boldsymbol{U}_{h}^{n}-\boldsymbol{u}^{n}\right\|_{L^{6}\left(\Omega ; \mathbb{R}^{3}\right)} \leq c\left|\boldsymbol{u}^{n}-\boldsymbol{U}_{h}^{n}\right|_{V_{h}^{2}\left(\Omega ; \mathbb{R}^{3}\right)},
\end{gathered}
$$

where we have used estimate (9.14) and the Sobolev inequality (9.30). Finally, employing Young's inequality, and estimate (4.13), we arrive at

$$
\begin{aligned}
\left|\mathcal{P}_{1}\right| \leq c\left(\delta, M_{0}, E_{0}, \underline{r}, \bar{r}\right. & \left.\left\|\left(\boldsymbol{U}, \nabla \boldsymbol{U}, \partial_{t} \boldsymbol{U}, \partial_{t} \nabla \boldsymbol{U}\right)\right\|_{L^{\infty}\left(Q_{T}, \mathbb{R}^{15}\right)}\right) \\
& \times\left(k \mathcal{E}\left(\varrho^{0}, \boldsymbol{u}^{0} \mid r^{0}, \boldsymbol{U}^{0}\right)+k \sum_{n=1}^{m} \mathcal{E}\left(\varrho^{n}, \boldsymbol{u}^{n} \mid r^{n}, \boldsymbol{U}^{n}\right)\right)+\delta k \sum_{n=1}^{m}\left|\boldsymbol{u}^{n}-\boldsymbol{U}_{h}^{n}\right|_{V_{h}^{2}\left(\Omega ; \mathbb{R}^{3}\right)}^{2}
\end{aligned}
$$

with any $\delta>0$.

Step 2: $\operatorname{Term} \mathcal{P}_{2}$. We write $\mathcal{P}_{2}=k \sum_{n=1}^{m} \mathcal{P}_{2}^{n}$ where Lemma 7.2 and the Hölder inequality yield, similarly as in the previous step,

$$
\begin{aligned}
\left|\mathcal{P}_{2}^{n}\right| & \leq c\left(\underline{r}, \bar{r},\|\nabla \boldsymbol{U}\|_{L^{\infty}\left(Q_{T} ; \mathbb{R}^{9}\right)}\right) \times \\
& \sum_{K \in \mathcal{T}} \sum_{\sigma \in \mathcal{E}(K)}|\sigma| h\left(E^{1 / 2}\left(\varrho_{\sigma}^{n, \text { up }}, \hat{r}_{\sigma}^{n, \text { up }}\right)+E^{2 / 3}\left(\varrho_{\sigma}^{n, \text { up }}, \hat{r}_{\sigma}^{n, \text { up }}\right)\left|\hat{\boldsymbol{U}}_{h, \sigma}^{n, \text { up }}\right|\left|\hat{\boldsymbol{U}}_{h, \sigma}^{n, \text { up }}-\hat{\boldsymbol{u}}_{\sigma}^{n, \text { up }}\right|\right. \\
& \leq c\left(\underline{r}, \bar{r},\|(\boldsymbol{U}, \nabla \boldsymbol{U})\|_{L^{\infty}\left(Q_{T} ; \mathbb{R}^{12}\right)}\right)\left[\left(\sum_{K \in \mathcal{T}} \sum_{\sigma \in \mathcal{E}(K)}|\sigma| h\left(E\left(\varrho_{\sigma}^{n, \mathrm{up}} \mid \hat{r}_{\sigma}^{n, \mathrm{up}}\right)\right)^{1 / 2}\right.\right. \\
& \left.+\left(\sum_{K \in \mathcal{T}} \sum_{\sigma \in \mathcal{E}(K)}|\sigma| h E\left(\varrho_{\sigma}^{n, \text { up }} \mid \hat{r}_{\sigma}^{n, \mathrm{up}}\right)\right)^{2 / 3}\right] \times\left(\sum_{K \in \mathcal{T}} \sum_{\sigma \in \mathcal{E}(K)}|\sigma| h\left|\hat{\boldsymbol{U}}_{h, \sigma}^{n, \text { up }}-\hat{\boldsymbol{u}}_{\sigma}^{n, \text { up }}\right|^{6}\right)^{1 / 6}
\end{aligned}
$$

provided $\gamma \geq 3 / 2$. Next, we observe that the contribution of the face $\sigma=K \mid L$ to the sums $\sum_{K \in \mathcal{T}}$ $\sum_{\sigma \in \mathcal{E}(K)}|\sigma| h E\left(\varrho_{\sigma}^{n, \text { up }} \mid \hat{r}_{\sigma}^{n, \text { up }}\right)$ and $\sum_{K \in \mathcal{T}} \sum_{\sigma \in \mathcal{E}(K)}|\sigma| h\left|\hat{\boldsymbol{U}}_{h, \sigma}^{n, \text { up }}-\hat{\boldsymbol{u}}_{\sigma}^{n \text {,up }}\right|^{6}$ is less or equal than $2|\sigma| h\left(E\left(\varrho_{K}^{n} \mid \hat{r}_{K}^{n}\right)+\right.$ $\left.E\left(\varrho_{L}^{n} \mid \hat{r}_{L}^{n}\right)\right)$, and than $2|\sigma| h\left(\left|\boldsymbol{U}_{h, K}^{n}-\boldsymbol{u}_{K}^{n}\right|^{6}+\left|\boldsymbol{U}_{h, L}^{n}-\boldsymbol{u}_{L}^{n}\right|^{6}\right)$, respectively. Consequently, we get by the same reasoning as in the previous step, under assumption $\gamma \geq 3 / 2$,

$$
\left.\left|\mathcal{P}_{2}\right| \leq c\left(\delta, M_{0}, E_{0}, \underline{r}, \bar{r},\|(\boldsymbol{U}, \nabla \boldsymbol{U})\|_{L^{\infty}\left(Q_{T} ; \mathbb{R}^{12}\right)}\right) k \sum_{n=1}^{m} \mathcal{E}\left(\varrho^{n}, \boldsymbol{u}^{n} \mid r^{n}, \boldsymbol{U}^{n}\right)+\delta k \sum_{n=1}^{m} \mid \boldsymbol{u}^{n}-\boldsymbol{U}_{h}^{n}\right)\left.\right|_{V_{h}^{2}\left(\Omega ; \mathbb{R}^{3}\right)} ^{2}
$$


Step 3: Term $\mathcal{P}_{3}$. Since the pair $(r, \boldsymbol{U})$ satisfies continuity equation (1.1a) in the classical sense, we have for all $n=1, \ldots, N$,

$$
\left[\partial_{t} r\right]^{n}+\boldsymbol{U}^{n} \cdot \nabla r^{n}=-r^{n} \operatorname{div} \boldsymbol{U}^{n},
$$

where we recall that $\left[\partial_{t} r\right]^{n}(x)=\partial_{t} r\left(t_{n}, x\right)$ in accordance with (3.17). Using this identity we write

$$
\begin{aligned}
& \mathcal{P}_{3}^{n}=\mathcal{P}_{3,1}+\mathcal{P}_{3,2}, \quad \mathcal{P}_{3, i}=k \sum_{n=1}^{m} \mathcal{P}_{3, i}^{n}, \\
& \text { with } \mathcal{P}_{3,1}^{n}=-\sum_{K \in \mathcal{T}} \int_{K}\left(p\left(\varrho_{K}^{n}\right)-p^{\prime}\left(r_{K}^{n}\right)\left(\varrho_{K}^{n}-r_{K}^{n}\right)-p\left(r_{K}^{n}\right)\right) \operatorname{div} \boldsymbol{U}^{n} \mathrm{~d} x \\
& \text { and } \mathcal{P}_{3,2}^{n}=\sum_{K \in \mathcal{T}}\left[\int_{K} \frac{r_{K}^{n}-\varrho_{K}^{n}}{r_{K}^{n}} p^{\prime}\left(r_{K}^{n}\right)\left(\boldsymbol{u}^{n}-\boldsymbol{U}^{n}\right) \cdot \nabla r^{n} \mathrm{~d} x .\right.
\end{aligned}
$$

Now, we apply Lemma 7.2 in combination with assumption (1.6) to deduce

$$
\left|\mathcal{P}_{3,1}\right| \leq c\|\operatorname{div} \boldsymbol{U}\|_{L^{\infty}\left(Q_{T}\right)} k \sum_{n=1}^{m} \mathcal{E}\left(\varrho^{n}, \boldsymbol{u}^{n} \mid r^{n}, \boldsymbol{U}^{n}\right) .
$$

Finally, the same reasoning as in Step 2 leads to the estimate

$$
\begin{aligned}
\left|\mathcal{P}_{3,2}\right| & \leq h c\left(M_{0}, E_{0}, \underline{r}, \bar{r},\left|p^{\prime}\right|_{C([\underline{r}, \bar{r}])}\|(\nabla r, \nabla \boldsymbol{U})\|_{L^{\infty}\left(\Omega ; \mathbb{R}^{9}\right)}\right) \\
& +c\left(\delta,\left\|\underline{r}, \bar{r},\left|p^{\prime}\right|_{C([\underline{r}, \bar{r}])}\right\| \nabla r \|_{L^{\infty}\left(\Omega ; \mathbb{R}^{3}\right)}\right) k \sum_{n=1}^{m} \mathcal{E}\left(\varrho^{n}, \boldsymbol{u}^{n} \mid r^{n}, \boldsymbol{U}^{n}\right)+\delta k \sum_{n=1}^{m}\left|\boldsymbol{u}^{n}-\boldsymbol{U}_{h}^{n}\right|_{V_{h}^{2}\left(\Omega ; \mathbb{R}^{3}\right)}^{2}
\end{aligned}
$$

Gathering the formulae (8.1) and (8.3)-(8.6) with $\delta$ sufficiently small (with respect to $\mu$ ), we conclude the proof of Lemma 8.1

Finally, Lemma 8.1 in combination with the bound (4.13) yields

$$
\mathcal{E}\left(\varrho^{m}, \boldsymbol{u}^{m} \mid r^{m}, \boldsymbol{U}^{m}\right) \leq c\left[h^{A}+\sqrt{k}+k+\mathcal{E}\left(\varrho^{0}, \boldsymbol{u}^{0} \mid r^{0}, \boldsymbol{U}^{0}\right)\right]+c k \sum_{n=1}^{m-1} \mathcal{E}\left(\varrho^{n}, \boldsymbol{u}^{n} \mid r^{n}, \boldsymbol{U}^{n}\right)
$$

whence Theorem 3.1 is a direct consequence of the standard discrete version of Gronwall's lemma. Theorem 3.1 is thus proved.

\section{9 - Appendix: Fundamental auxiliary lemmas and estimates}

In this section we report several results related to the properties of the Sobolev spaces on tetrahedra and of the Crouzeix-Raviart (C-R) space. We refer to the book Brezzi, Fortin [3] for the general introduction to the subject.

We start with the inequalities that can be obtained by rescaling from the standard inequalities on a reference tetrahedron of size equivalent to one.

Lemma 9.1 ( Poincaré, Sobolev and interpolation inequalities on tetrahedra). Let $1 \leq p \leq \infty$. Let $\theta_{0}>0$ and $\mathcal{T}$ be a triangulation of $\Omega$ such that $\theta \geq \theta_{0}$ where $\theta$ is defined in (3.1). Then we have:

(1) Poincaré type inequalities on tetrahedra

Let $1 \leq p \leq \infty$. There exists $c=c\left(\theta_{0}, p\right)>0$ such that for all $K \in \mathcal{T}$ and for all $v \in W^{1, p}(K)$ we have

$$
\begin{gathered}
\left\|v-v_{K}\right\|_{L^{p}(K)} \leq c h\|\nabla v\|_{L^{p}(K)}, \\
\forall \sigma \in \mathcal{E}(K),\left\|v-v_{\sigma}\right\|_{L^{p}(K)} \leq \operatorname{ch}\|\nabla v\|_{L^{p}(K)} .
\end{gathered}
$$


(2) Sobolev type inequalities on tetrahedra

Let $1 \leq p<d$. There exists $c=c\left(\theta_{0}, p\right)>0$ such that for all $K \in \mathcal{T}$ and for all $v \in W^{1, p}(K)$ we have

$$
\begin{gathered}
\left\|v-v_{K}\right\|_{L^{p^{*}(K)}} \leq c\|\nabla v\|_{L^{p}(K)}, \\
\forall \sigma \in \mathcal{E}(K),\left\|v-v_{\sigma}\right\|_{L^{p^{*}(K)}} \leq c\|\nabla v\|_{L^{p}(K)},
\end{gathered}
$$

where $p^{*}=\frac{d p}{d-p}$.

(3) Interpolation inequalities on the tetrahedra

Let $1 \leq p<d$ and $p \leq q \leq p^{*}$. There exists $c=c\left(\theta_{0}, p\right)>0$ such that for all $K \in \mathcal{T}$ and $v \in W^{1, p}(K)$ we have

$$
\begin{gathered}
\left\|v-v_{K}\right\|_{L^{q}(K)} \leq c h^{\beta}\|\nabla v\|_{L^{p}\left(K ; \mathbb{R}^{d}\right)}, \\
\left\|v-v_{\sigma}\right\|_{L^{q}(K)} \leq c h^{\beta}\|\nabla v\|_{L^{p}\left(K ; \mathbb{R}^{d}\right)},
\end{gathered}
$$

where $\frac{1}{q}=\frac{\beta}{p}+\frac{1-\beta}{p^{*}}$.

Combining estimates (9.19.6) with the algebraic inequality

$$
\left(\sum_{i=1}^{L}\left|a_{i}\right|^{p}\right)^{1 / p} \leq\left(\sum_{i=1}^{L}\left|a_{i}\right|^{q}\right)^{1 / q}
$$

for all $\left(a=\left(a_{1}, \ldots, a_{L}\right) \in \mathbb{R}^{L}, 1 \leq q \leq p<\infty\right.$, we obtain the following corollaries.

Corollary 9.1 (Poincaré and Sobolev type inequalities on the Sobolev spaces). Under the assumptions of Lemma 9.1, we have:

(1) Poincaré type inequalities on the domain $\Omega$

Let $1 \leq p \leq \infty$. There exists $c=c\left(\theta_{0}, p\right)>0$ such that for all $v \in W^{1, p}(\Omega)$ we have

$$
\begin{aligned}
& \|v-\hat{v}\|_{L^{p}(\Omega)} \equiv\left(\sum_{K \in \mathcal{T}}\left\|v-v_{K}\right\|_{L^{p}(K)}^{p}\right)^{1 / p} \leq \operatorname{ch}\|\nabla v\|_{L^{p}\left(\Omega ; \mathbb{R}^{d}\right)}, \\
& \left(\sum_{K \in \mathcal{T}} \sum_{\sigma \in \mathcal{E}(K)}\left\|v-v_{\sigma}\right\|_{L^{p}(K)}^{p}\right)^{1 / p} \leq c h\|\nabla v\|_{L^{p}\left(\Omega ; \mathbb{R}^{d}\right)}
\end{aligned}
$$

where $\hat{v}$ and $v_{\sigma}$ are defined by (3.7) and (3.8).

(2) Sobolev type inequalities on the domain $\Omega$

Let $1 \leq p<d$. There exists $c=c\left(\theta_{0}, p\right)>0$ such that for all $v \in W^{1, p}(\Omega)$ we have

$$
\begin{gathered}
\|v-\hat{v}\|_{L^{p^{*}}(\Omega)} \leq c\|\nabla v\|_{L^{p}(\Omega)}, \\
\left(\sum_{K \in \mathcal{T}} \sum_{\sigma \in \mathcal{E}(K)}\left\|v-v_{\sigma}\right\|_{L^{p^{*}}(K)}^{p^{*}}\right)^{1 / p^{*}} \leq c\|\nabla v\|_{L^{p}\left(\Omega ; \mathbb{R}^{d}\right)} .
\end{gathered}
$$

(3) Interpolation inequalities on the domain $\Omega$

Let $1 \leq p<d$ and $p \leq q \leq p^{*}$. There exists $c=c\left(\theta_{0}, p\right)>0$ such that for all $v \in W^{1, p}(\Omega)$ we have

$$
\begin{gathered}
\|v-\hat{v}\|_{L^{q}(\Omega)} \leq c h^{\beta}\|\nabla v\|_{L^{p}(\Omega)}, \\
\left(\sum_{K \in \mathcal{T}} \sum_{\sigma \in \mathcal{E}(K)}\left\|v-v_{\sigma}\right\|_{L^{q}(K)}^{q}\right)^{\frac{1}{q}} \leq c h^{\beta}\|\nabla v\|_{L^{p}\left(\Omega ; \mathbb{R}^{d}\right)},
\end{gathered}
$$

where $\frac{1}{q}=\frac{\beta}{p}+\frac{1-\beta}{p^{*}}$. 
Corollary 9.2 (Poincaré and Sobolev type inequalities on $V_{h}$ ). Under assumptions of Lemma 9.1, there holds:

(1) Poincaré type inequality in $V_{h}(\Omega):$ Let $1 \leq p<\infty$. There exists $c=c\left(\theta_{0}, p\right)$ such that for all $v \in V_{h}$,

$$
\begin{gathered}
\|v-\hat{v}\|_{L^{p}(\Omega)} \leq c h|v|_{V_{h}^{p}(\Omega)}, \\
\left(\sum_{K \in \mathcal{T}} \sum_{\sigma \in \mathcal{E}(K)}\left\|v-v_{\sigma}\right\|_{L^{p}(K)}^{p}\right)^{\frac{1}{p}} \leq \operatorname{ch}|v|_{V_{h}^{p}(\Omega)} .
\end{gathered}
$$

(2) Sobolev type inequality in $V_{h}(\Omega)$ : Let $1 \leq p<d$. There exists $c=c\left(\theta_{0}, p\right)$ such that for all $v \in V_{h}(\Omega)$,

$$
\begin{gathered}
\|v-\hat{v}\|_{L^{p^{*}}(\Omega)} \leq c|v|_{V_{h}^{p}(\Omega)}, \\
\left(\sum_{K \in \mathcal{T}} \sum_{\sigma \in \mathcal{E}(K)}\left\|v-v_{\sigma}\right\|_{L^{p^{*}}(K)}^{p^{*}}\right)^{\frac{1}{p^{*}}} \leq c|v|_{V_{h}^{p}(\Omega)} .
\end{gathered}
$$

(3) Interpolation type inequalities in $V_{h}(\Omega)$

Let $1 \leq p<d$ and $p \leq q \leq p^{*}$. There exists $c=c\left(\theta_{0}, p\right)>0$ such that for all $v \in V_{h}(\Omega)$ we have

$$
\begin{gathered}
\|v-\hat{v}\|_{L^{q}(\Omega)} \leq c h^{\beta}|v|_{V_{h}^{p}(\Omega)}, \\
\left(\sum_{K \in \mathcal{T}} \sum_{\sigma \in \mathcal{E}(K)}\left\|v-v_{\sigma}\right\|_{L^{q}(K)}^{q}\right)^{\frac{1}{q}} \leq c h^{\beta}|v|_{V_{h}^{p}(\Omega)},
\end{gathered}
$$

where $\frac{1}{q}=\frac{\beta}{p}+\frac{1-\beta}{p^{*}}$.

The next fundamental lemma deals with the properties of the projection $v_{h}$ defined by (3.9).

Lemma 9.2 (Projection on $V_{h}$ ). Let $\theta_{0}>0$ and $\mathcal{T}$ be a triangulation of $\Omega$ such that $\theta \geq \theta_{0}$ where $\theta$ is defined in (3.1).

(1) Approximation estimates on the tetrahedra

Let $1 \leq p \leq \infty$. There exists $c=c\left(\theta_{0}, p\right)>0$ such that

$$
\begin{gathered}
\forall v \in W_{0}^{1, p}(\Omega) \cap L^{\infty}(\Omega), \forall K \in \mathcal{T},\left\|v_{h}\right\|_{L^{\infty}(K)} \leq c\|v\|_{L^{\infty}(K)}, \\
\forall v \in W_{0}^{1, p} \cap W^{s, p}(\Omega), \forall K \in \mathcal{T},\left\|v-v_{h}\right\|_{L^{p}(K)} \leq c h^{s}\left\|\nabla^{s} v\right\|_{L^{p}\left(K ; \mathbb{R}^{d^{s}}\right)}, \\
\left\|\nabla\left(v-v_{h}\right)\right\|_{L^{p}\left(K ; \mathbb{R}^{d}\right)} \leq c h^{s-1}\left\|\nabla^{s} v\right\|_{L^{p}\left(K ; \mathbb{R}^{s}\right)}, s=1,2 .
\end{gathered}
$$

(2) Preservation of divergence

$$
\forall \boldsymbol{v} \in W_{0}^{1,2}\left(\Omega, \mathbb{R}^{d}\right), \forall q \in L_{h}(\Omega), \sum_{K \in \mathcal{T}} \int_{K} q \operatorname{div} \boldsymbol{v}_{h} \mathrm{~d} x=\int_{\Omega} q \operatorname{div} \boldsymbol{v} \mathrm{d} x
$$

(3) Approximation estimates of the Poincaré type on the whole domain

Let $1 \leq p<\infty$. There exists $c=c\left(\theta_{0}, p\right)>0$ such that for all $v \in W_{0}^{1, p}(\Omega)$,

$$
\left\|v-v_{h}\right\|_{L^{p}(\Omega)} \leq \operatorname{ch}\|\nabla v\|_{L^{p}\left(\Omega ; \mathbb{R}^{d}\right)} .
$$


(4) Approximation estimates of the Sobolev type on the whole domain

Let $1 \leq p<d$. There exists $c=c\left(\theta_{0}, p\right)>0$ such that for all $v \in W_{0}^{1, p}(\Omega)$,

$$
\left\|v-v_{h}\right\|_{L^{p^{*}}(\Omega)} \leq c\|\nabla v\|_{L^{p}\left(\Omega ; \mathbb{R}^{d}\right)} .
$$

Statement (2) of Lemma 9.2 is proved in [6], where one can find also the proof of item (1) for $p=2$. We present here the proof of statements (1), (3), (4) for arbitrary $p$ for the reader's convenience, since a straightforward reference is not available.

Proof. Step 1: We start with some generalities. First we complete the Crouzeix-Raviart basis (3.6) by functions $\phi_{\sigma}$ indexed also with $\sigma \in \mathcal{E}_{\text {ext }}$ saying

$$
\frac{1}{\left|\sigma^{\prime}\right|} \int_{\sigma^{\prime}} \phi_{\sigma} \mathrm{d} S=\delta_{\sigma, \sigma^{\prime}}, \quad\left(\sigma, \sigma^{\prime}\right) \in \mathcal{E}^{2}
$$

and observe that

$$
\sum_{\sigma \in \mathcal{E}(K)} \phi_{\sigma}(x)=1 \text { for any } x \in K
$$

A scaling argument yields

$$
\left\|\phi_{\sigma}\right\|_{L^{\infty}(\Omega)} \leq c\left(\theta_{0}\right), h\left\|\nabla \phi_{\sigma}\right\|_{L^{\infty}\left(\Omega ; \mathbb{R}^{d}\right)} \leq c\left(\theta_{0}\right) .
$$

Second, we define the projection $v \rightarrow v_{h}$ for any $v \in W^{1, p}(\Omega)$ by saying

$$
v_{h}=\sum_{\sigma \in \mathcal{E}} v_{\sigma} \phi_{\sigma}
$$

We notice that if $v \in W_{0}^{1, p}(\Omega)$ then $v_{h}$ coincides with (3.5). Moreover,

$$
v_{h}=v \text { for any affine function } v \text {. }
$$

Third, due to the density argument, it is enough to show the remaining statements (1), (3), (4) for $v \in W_{0}^{1, p}(\Omega) \cap W^{s, \infty}(\Omega), s=1,2$, according to the case.

Step 2: We realize that $\operatorname{supp} \phi_{\sigma}=K \cup L$ and derive (9.20) directly by employing representation (3.9), definition of $v_{\sigma}$ and estimate (9.27).

We denote by $x_{K}=\frac{1}{|K|} \int_{K} x \mathrm{~d} x$ the center of gravity of the tetrahedron $K$. We calculate by using (9.28) and the first order Taylor formula

$$
\begin{gathered}
v(x)-v_{h}(x)=v(x)-v\left(x_{K}\right)-\left[v-v\left(x_{K}\right)\right]_{h}(x) \\
=\left(x-x_{K}\right) \cdot \int_{0}^{1} \nabla v\left(x_{K}+t\left(x-x_{K}\right)\right) \mathrm{d} t-\sum_{\sigma \in \mathcal{E}(K)} \phi_{\sigma}(x) \frac{1}{|\sigma|} \int_{\sigma}\left[\left(x-x_{K}\right) \cdot \int_{0}^{1} \nabla v\left(x_{K}+t\left(x-x_{K}\right)\right) \mathrm{d} t\right] \mathrm{d} S,
\end{gathered}
$$

where $x \in K$. This formula yields immediately the upper bound stated in (9.21) $s=1$ if $p=\infty$. If $1 \leq p<\infty$ we calculate the upper bound of the $L^{p}$-norm of each term at the right-hand side separately by using (9.27), Fubini's theorem, Hölder's inequality and the change of variables $y=x_{K}+t\left(x-x_{K}\right)$ together with the convexity of $K$.

The same reasoning can be applied to prove (9.21) $s=2$. Indeed, we observe that

$$
v(x)-v_{h}(x)=v(x)-\left(x-x_{K}\right) \cdot \nabla v\left(x_{K}\right)-v\left(x_{K}\right)-\left[v-\left(x-x_{K}\right) \cdot \nabla v\left(x_{K}\right)-v\left(x_{K}\right)\right]_{h}(x)
$$


by virtue of (9.28). Now we apply to the right hand side of the last expression the second order Taylor formula in the integral form, and proceed exactly as described before.

Finally, one applies the same straightforward argumentation to get (9.22). This completes the proof of statement (1).

Step 3: Statement (3) follows easily from $(9.21)_{s=1}$ and the algebraic inequality (9.7).

Step 4: We use (9.26) and (9.28) to write

$$
v(x)-v_{h}(x)=\sum_{\sigma \in \mathcal{E}(K)}\left(v(x)-v_{\sigma}\right) \phi_{\sigma}(x), \quad x \in K
$$

whence

$$
\left\|v-v_{h}\right\|_{L^{p^{*}}(K)} \leq c\|\nabla v\|_{L^{p}\left(K ; \mathbb{R}^{d}\right)}
$$

where we have used the Sobolev inequality (9.4) on the tetrahedron $K \in \mathcal{T}$ and the $L^{\infty}$-bound (9.27). We conclude the proof of statement (4) by using the relation (9.7). The proof of Lemma 9.2 is complete.

The following corollary is a direct consequence of (9.22).

Corollary 9.3 (Continuity of the projection onto $V_{h}$ ). Under assumptions of Lemma 9.2, there exists $c=c\left(\theta_{0}, p\right)>0$ such that

$$
\forall v \in W_{0}^{1, p}(\Omega),\left|v_{h}\right|_{V_{h}^{p}(\Omega)} \leq c\|\nabla v\|_{L^{p}\left(\Omega ; \mathbb{R}^{d}\right)},
$$

where $1 \leq p<\infty$.

Although the non conforming finite element space $V_{h}$ is not a subspace of any Sobolev space, its elements enjoy the Sobolev type inequalities. This important fact is formulated in the next lemma.

Lemma 9.3 (Sobolev inequality on $V_{h}$ ). Let $\Omega$ be a bounded domain of $\mathbb{R}^{d}$. Let $\mathcal{T}$ be a triangulation of the domain $\Omega$ in simplices such that $\theta \geq \theta_{0}>0$ where $\theta$ is defined in (3.1). Then we have:

(1) Sobolev inequality in $V_{h}(\Omega)$ (case $\left.1 \leq p<d\right)$ :

There exists $c=c\left(\theta_{0}, p\right)$ such that for all $v \in V_{h}(\Omega)$,

$$
\|v\|_{L^{p^{*}}(\Omega)} \leq c|v|_{V_{h}^{p}(\Omega)}
$$

(2) Sobolev inequality in $V_{h}(\Omega)$, case $p \geq d$

Let $1 \leq q<\infty$. There here exits $c=c\left(\theta_{0}, p, q\right)>0$ such that forall $v \in V_{h}(\Omega)$,

$$
\|v\|_{L^{q}(\Omega)} \leq c|v|_{V_{h}^{p}(\Omega)}
$$

Proof. Step 1 Let $1 \leq r \leq \alpha<\infty$. Let $u \in V_{h}$. We call $v$ the element of $V_{h}$ such that $v_{\sigma}=\left|u_{\sigma}\right|^{\alpha}$. Then there exists $C$ only depending on $d, r, \alpha$ such that

$$
\|u\|_{L^{r}(\Omega)}^{\alpha} \leq\|u\|_{L^{\frac{r}{\alpha}}(\Omega)} .
$$

To prove (9.32) we remark that, using a change of variable, it is enough to show to prove the existence of $C$ for only the unit symplex $\hat{K}$. Let $u \in \mathbb{P}_{1}(\hat{K})$ and we call $v$ the element of $\mathbb{P}_{1}(\hat{K})$ such that $v_{\sigma}=\left|u_{\sigma}\right|^{\alpha}$. Let $T(u)=\|u\|_{L^{r}(\hat{K})}$ and $S(u)=\|u\|_{L^{\frac{r}{\alpha}(\hat{K})}}^{\frac{1}{\alpha}}$. These two functions are continuous, homogeneous of degree 1 and non zero if $u \neq 0$. Since $\mathbb{P}_{1}(\hat{K})$ is a finite dimensional space, we can choose a norm on $\mathbb{P}_{1}(\hat{K})$ and take $C=\left(\frac{M}{m}\right)^{\alpha}$ where $M=\max \left\{T(u),\|u\|_{\mathbb{P}_{1}(\hat{K})}=1\right\}$ and $m=\min \left\{T(u),\|u\|_{\mathbb{P}_{1}(\hat{K})}=1\right\}$. 
Step 2: Proof for $p=1$.

We set $u=0$ outside $\Omega$. For $\sigma \in \mathcal{E}_{\text {int }}, \sigma=K \mid L$, we set $|[u(x)]|=\left|u_{K}(x)-u_{L}(x)\right|$ for $x \in \sigma$. For $\sigma \in \mathcal{E}_{\text {ext }} \cap \mathcal{E}(K)$, we set $|[u(x)]|=\left|u_{K}(x)\right|$ for $x \in \sigma$. We first remark that there exists $C_{1,1}$ and $C_{1,2}$ only depending on $d$ such that

$$
\|u\|_{L^{\frac{d}{d-1}(\Omega)}} \leq C_{1,1}\|u\|_{B V\left(\mathbb{R}^{d}\right)} \leq C_{1,2}\left\|\nabla_{h} u\right\|_{L^{1}(\Omega)}+C_{1,2} \sum_{\sigma \in \mathcal{E}} \int_{\sigma}|[u]| \mathrm{d} S .
$$

We now prove that there exits $C_{1,3}$ only depending on $d$ and $\theta_{0}$ such that

$$
\sum_{\sigma \in \mathcal{E}} \int_{\sigma}|[u]| \mathrm{d} S \leq C_{1,3}\left\|\nabla_{h} u\right\|_{L^{1}(\Omega)}
$$

Let $K \in \mathcal{T}$ and $\sigma \in \mathcal{E}(K)$. Let $x_{\sigma}$ be the center of mass of $\sigma$. We have, with $u_{K}=u$ in $K$,

$$
u_{K}(x)-u\left(x_{\sigma}\right)=\int_{0}^{1} \nabla u_{K} \cdot\left(x-x_{\sigma}\right) \mathrm{d} x
$$

Then if $\sigma=K \mid L$ we have

$$
\left|u_{K}(x)-u_{L}(x)\right| \leq h_{\sigma}\left(\left|\nabla u_{K}\right|+\left|\nabla u_{L}\right|\right) .
$$

Integrating this inequality on $\sigma$ gives

$$
\int_{\sigma}|[u]| \mathrm{d} S \leq|\sigma| h_{\sigma}\left(\left|\nabla u_{K}\right|+\left|\nabla u_{L}\right|\right) \leq \frac{2}{\theta_{0}^{d}}\left(\|\nabla u\|_{L^{1}(K)}+\|\nabla u\|_{L^{1}(L)}\right) .
$$

Similarly for $\sigma \in \mathcal{E}_{\text {ext }} \cap \mathcal{E}(K)$ we have

$$
\int_{\sigma}|[u]| \mathrm{d} S \leq \frac{2}{\theta_{0}^{d}}\|\nabla u\|_{L^{1}(K)}
$$

Then there exists $C_{1,3}=C\left(d, \theta_{0}\right)$ such that

$$
\sum_{i, \sigma \in \mathcal{E}} \int_{\sigma}|[u]| \mathrm{d} S \leq C_{1,3}\left\|\nabla_{h} u\right\|_{L^{1}(\Omega)} .
$$

and then,

$$
\|u\|_{L^{1^{*}}(\Omega)} \leq c\left(d, \theta_{0}\right)\left\|\nabla_{h} u\right\|_{L^{1}(\Omega)} .
$$

Step 3: Proof for $1<p<d$.

Let $1<p<d$ and $p^{*}=\frac{p d}{d-p}$ and let $u \in V_{h}$. We set $u=0$ outside $\Omega$. Let $\alpha=\frac{p(d-1)}{d-p}$, so that $\alpha>1$ and $\alpha 1^{*}=p^{*}$. We call $v$ the element of $V_{h}$ such that $v_{\sigma}=\left|u_{\sigma}\right|^{\alpha}$ for $\sigma \in \mathcal{E}$. One has $v \neq|u|^{\alpha}$ but there exits $C_{2,1}$ only depending on $d$ and $p$ (see lemma 9.32) such that

$$
\|u\|_{L^{p^{*}}(\Omega)}^{\alpha} \leq C_{2,1}\|v\|_{L^{1^{*}}(\Omega)} \leq c\left(d, p, \theta_{0}\right)\left\|\nabla_{h} v\right\|_{L^{1}(\Omega)} .
$$

Moreover using a scalling argument we obtain

$$
\left\|\nabla_{h} v\right\|_{L^{1}(K)} \leq c\left(d, p, \theta_{0}\right) \sum_{\sigma \in \mathcal{E}(K)}\left|u_{\sigma}\right|^{\alpha-1}\left|\nabla u_{K}\right||K| .
$$

Then, using Hölder Inequality, we have, with $q=\frac{p}{p-1}\left(\right.$ so that $\left.q(\alpha-1)=p^{*}\right)$,

$$
\left\|\nabla_{h} v\right\|_{L^{1}(K)} \leq c\left(d, p, \theta_{0}\right)\|\nabla u\|_{L^{p}(K)}\|u\|_{L^{p^{*}(K)}}^{\frac{p^{*}}{q}} .
$$


Summing on $K \in \mathcal{T}$ we obtain

Step 4: Proof for $p \geq d$.

$$
\|u\|_{L^{p^{*}}(\Omega)} \leq C_{2}\left\|\nabla_{h} u\right\|_{L^{p}(\Omega)} .
$$

Let $1 \leq q<\infty$. There exists $r=r(d, q)$ such that $r<d$ and $r^{*} \geq q$. We have

$$
\|u\|_{L^{r^{*}}(\Omega)} \leq c\left(r, d, q, \theta_{0}\right)\left\|\nabla_{h} u\right\|_{L^{r}(\Omega)} .
$$

Moreover

$$
\|u\|_{L^{q}(\Omega)} \leq|\Omega|^{\frac{1}{q}-\frac{1}{r^{*}}}|| u\left\|_{L^{r^{*}}(\Omega)} \leq c\left(d, q, \theta_{0}\right)|\Omega|^{\frac{1}{q}-\frac{1}{r^{*}}}\right\| \nabla_{h} u \|_{L^{r}(\Omega)}
$$

and

Finally

$$
\left\|\nabla_{h} u\right\|_{L^{r}(\Omega)} \leq|\Omega|^{\frac{1}{r}-\frac{1}{p}}\left\|\nabla_{h} u\right\|_{L^{p}(\Omega)} .
$$

$$
\|u\|_{L^{q}(\Omega)} \leq c\left(\Omega, d, p, q, \theta_{0}\right)\left\|\nabla_{h} u\right\|_{L^{p}(\Omega)} .
$$

A Combination of Lemma 9.3 with estimates (9.14), (9.16) and the Hölder inequality yields the following corollary.

Corollary 9.4 (Estimates of the norms of mean values). We have under the assumptions of Lemma 9.3;

(1) Poincaré type inequality involving mean values on tetrahedra

There exists $c=c\left(\theta_{0}, p\right)$ such that for all $v \in V_{h}$,

$$
\|\hat{v}\|_{L^{p}(\Omega)} \equiv\left(\sum_{K \in \mathcal{T}}|K|\left|v_{K}\right|^{p}\right)^{1 / p} \leq c\left(\|v\|_{L^{p}(\Omega)}+h|v|_{V_{h}^{p}(\Omega)}\right) .
$$

(2) Sobolev type inequality involving mean values on tetrahedrons

Let $1 \leq p<d$, there exists $c=c\left(\theta_{0}, p\right)$ such that for all $v \in V_{h}$,

$$
\|\hat{v}\|_{L^{p^{*}}(\Omega)} \equiv\left(\sum_{K \in \mathcal{T}}\left|K \| v_{K}\right|^{p^{*}}\right)^{1 / p^{*}} \leq c\left(\|v\|_{L^{p^{*}}(\Omega)}+|v|_{V_{h}^{p}}\right) .
$$

Note that the Last but not least, we recall a result on equivalence of norms in the space $V_{h}(\Omega)$ which is a consequence of a discrete Poincaré inequality on the broken Sobolev space $V_{h}$ [33, proposition 4.13].

Lemma 9.4 (Discrete and continuous norms in $V_{h}$ ). Let $1 \leq p<\infty$. Let $\theta_{0}>0$ and $\mathcal{T}$ be a triangulation of $\Omega$ such that $\theta \geq \theta_{0}$ where $\theta$ is defined in (3.1). Then the norms

$$
\left(\sum_{\sigma \in \mathcal{E}_{\text {int }}}|\sigma| h\left|v_{\sigma}\right|^{p}\right)^{1 / p} \text { and }\|v\|_{L^{p}(\Omega)}^{p}
$$

are equivalent on $V_{h}(\Omega)$ uniformly with respect to $h>0$.

The last lemma in this overview deals with the estimates of jumps over faces. The reader can consult [8, Lemma 3.32] or [18, Lemma 2.2] for its proof.

Lemma 9.5 (Jumps over faces in the Crouzeix-Raviart space). Let $\theta_{0}>0$ and $\mathcal{T}$ be a triangulation of $\Omega$ such that $\theta \geq \theta_{0}$ where $\theta$ is defined in (3.1). Then there exists $c=c\left(\theta_{0}\right)>0$ such that for all $v \in V_{h}(\Omega)$,

$$
\sum_{\sigma \in \mathcal{E}} \frac{1}{h} \int_{\sigma}[v]_{\sigma, n_{\sigma}}^{2} \mathrm{~d} S \leq c|v|_{V_{h}^{2}(\Omega)}^{2},
$$

where $[v]_{\sigma, \boldsymbol{n}_{\sigma}}$ is a jump of $v$ with respect to a normal $\boldsymbol{n}_{\sigma}$ to the face $\sigma$,

$$
\forall x \in \sigma=K \mid L \in \mathcal{E}_{\mathrm{int}}, \quad[v]_{\sigma, \boldsymbol{n}_{\sigma}}(x)=\left\{\begin{array}{c}
\left.v\right|_{K}(x)-\left.v\right|_{L}(x) \text { if } \boldsymbol{n}_{\sigma}=\boldsymbol{n}_{\sigma, K} \\
\left.v\right|_{L}(x)-\left.v\right|_{K}(x) \text { if } \boldsymbol{n}_{\sigma}=\boldsymbol{n}_{\sigma, L}
\end{array}\right.
$$

and

$$
\forall x \in \sigma \in \mathcal{E}_{\mathrm{ext}}, \quad[v]_{\sigma, \boldsymbol{n}_{\sigma}}(x)=v(x), \text { with } \boldsymbol{n}_{\sigma} \text { an exterior normal to } \partial \Omega
$$




\section{9 - References}

[1] R. Babik, R. Herbin, W. Kheriji, and J.-C. Latché. Discretization of the viscous dissipation term with the mac scheme. In Finite Volumes for Complex Applications VI - Problems and Perspectives - Prague, Czech Republic, 2011.

[2] P. Bella, E. Feireisl, B. J. Jin, and A. Novotný. Dimensional reduction for compressible viscous fluids. Preprint IM-2013-21, http://www.math.cas.cz, 2013.

[3] F. Brezzi and M. Fortin. Mixed and hybrid finite elements methods. Springer series in computational mathematics.

[4] C. Cancès, H. Mathis, and N. Seguin. Relative entropy for the finite volume approximation of strong solutions to systems of conservation laws. HAL : hal-00798287, 2013.

[5] P. G. Ciarlet. Basic error estimates for elliptic problems. In P. Ciarlet and J. Lions, editors, Handbook of Numerical Analysis, Volume II, pages 17-351. North Holland, 1991.

[6] M. Crouzeix and P. Raviart. Conforming and nonconforming finite element methods for solving the stationary Stokes equations. RAIRO Série Rouge, 7:33-75, 1973.

[7] C. Dafermos. The second law of thermodynamics and stability. Arch. Rational Mech. Anal., 70:167$179,1979$.

[8] A. Ern and J.-L. Guermond. Theory and practice of finite elements, volume 159 of Applied Mathematical Sciences. Springer-Verlag, New York, 2004.

[9] R. Eymard, T. Gallouët, and R. Herbin. Finite volume methods. In P. G. Ciarlet and J.-L. Lions, editors, Techniques of Scientific Computing, Part III, Handbook of Numerical Analysis, VII, pages 713-1020. North-Holland, Amsterdam, 2000.

[10] R. Eymard, T. Gallouët, R. Herbin, and J.-C. Latché. Convergence of the MAC scheme for the compressible Stokes equations. SIAM Journal on Numerical Analysis, 48:2218-2246, 2010.

[11] R. Eymard, T. Gallouët, R. Herbin, and J. C. Latché. A convergent finite element-finite volume scheme for the compressible Stokes problem. II. The isentropic case. Math. Comp., 79(270):649-675, 2010 .

[12] E. Feireisl, B. J. Jin, and A. Novotný. Relative entropies, suitable weak solutions, and weak-strong uniqueness for the compressible Navier-Stokes system. J. Math. Fluid Mech., 14(4):717-730, 2012.

[13] E. Feireisl and A. Novotný. Weak-strong uniqueness property for the full Navier-Stokes-Fourier system. Arch. Ration. Mech. Anal., 204(2):683-706, 2012.

[14] E. Feireisl, A. Novotný, and H. Petzeltová. On the existence of globally defined weak solutions to the Navier-Stokes equations. J. Math. Fluid Mech., 3(4):358-392, 2001.

[15] E. Feireisl, A. Novotný, and Y. Sun. Suitable weak solutions to the Navier-Stokes equations of compressible viscous fluids. Indiana Univ. Math. J., 60(2):611-631, 2011.

[16] A. Fettah and T. Gallouët. Numerical approximation of the general compressible Stokes problem. IMA J. Numer. Anal., 33(3):922-951, 2013.

[17] T. Gallouët, L. Gastaldo, R. Herbin, and J.-C. Latché. An unconditionally stable pressure correction scheme for the compressible barotropic Navier-Stokes equations. M2AN Math. Model. Numer. Anal., 42(2):303-331, 2008. 
[18] T. Gallouët, R. Herbin, and J.-C. Latché. A convergent finite element-finite volume scheme for the compressible Stokes problem. I. The isothermal case. Math. Comp., 78(267):1333-1352, 2009.

[19] T. Gallouët, R. Herbin, D. Maltese, and A. Novotný. Error estimate for a numerical approximation to the compressible barotropic navier-stokes equations. ArXiv:, 2015.

[20] L. Gastaldo, R. Herbin, W. Kheriji, C. Lapuerta, and J.-C. Latché. Staggered discretizations, pressure correction schemes and all speed barotropic flows. In Finite volumes for complex applications. VI. Problems 85 perspectives. Volume 1, 2, volume 4 of Springer Proc. Math., pages 839-855. Springer, Heidelberg, 2011.

[21] L. Gastaldo, R. Herbin, and J.-C. Latché. An unconditionally stable finite element-finite volume pressure correction scheme for the drift-flux model. M2AN Math. Model. Numer. Anal., 44(2):251287,2010 .

[22] F. Harlow and J. Welsh. Numerical calculation of time-dependent viscous incompressible flow of fluid with free surface. Physics of Fluids, 8:2182-2189, 1965.

[23] R. Herbin, J.-C. Latché, and K. Mallem. Convergence of the MAC scheme for the steady-state incompressible Navier-Stokes equations on non-uniform grids. Proceedings of Finite Volumes for Complex Applications VII - Problems and Perspectives - Berlin, Germany, 2014.

[24] K. H. Karlsen and T. K. Karper. A convergent nonconforming finite element method for compressible Stokes flow. SIAM J. Numer. Anal., 48(5):1846-1876, 2010.

[25] K. H. Karlsen and T. K. Karper. Convergence of a mixed method for a semi-stationary compressible Stokes system. Math. Comp., 80(275):1459-1498, 2011.

[26] K. H. Karlsen and T. K. Karper. A convergent mixed method for the Stokes approximation of viscous compressible flow. IMA J. Numer. Anal., 32(3):725-764, 2012.

[27] T. K. Karper. A convergent FEM-DG method for the compressible Navier-Stokes equations. Numer. Math., 125(3):441-510, 2013.

[28] W. Kheriji, R. Herbin, and J.-C. Latché. Pressure correction staggered schemes for barotropic one-phase and two-phase flows. Comput. Ef Fluids, 88:524-542, 2013.

[29] J. Li and S. Sun. The superconvergence phenomenon and proof of the mac scheme for the stokes equations on non-uniform rectangular meshes. J. Sci. Comput., on line first:1-22, 2014.

[30] P.-L. Lions. Mathematical topics in fluid mechanics. Vol. 2, volume 10 of Oxford Lecture Series in Mathematics and its Applications. The Clarendon Press, Oxford University Press, New York, 1998. Compressible models, Oxford Science Publications.

[31] D. Maltese and A. Novotny. Compressible Navier-Stokes equations on thin domains. Journal of Mathematical Fluid Mechanics, 16:571-594, 2014.

[32] N. Masmoudi. Incompressible, inviscid limit of the compressible Navier-Stokes system. Ann. Inst. H. Poincaré Anal. Non Linéaire, 18(2):199-224, 2001.

[33] R. Temam. Navier-Stokes equations, volume 2 of Studies in Mathematics and its Applications. North-Holland Publishing Co., Amsterdam, third edition, 1984. Theory and numerical analysis, With an appendix by F. Thomasset.

[34] S. Wang, J. Yang, and D. Luo. Convergence of compressible Euler-Poisson system to incompressible Euler equations. Appl. Math. Comput., 216(11):3408-3418, 2010. 
[35] V. Yovanovic. An error estimate for a numerical scheme for the compressible Navier-Stokes system. Kragujevac J. Math., 30(1):263-275, 2007. 